\title{
THE STRUCTURE OF CLASSICAL BULGES AND PSEUDOBULGES: THE LINK BETWEEN PSEUDOBULGES AND SÉRSIC INDEX
}

\author{
DAVID B. FISHER ${ }^{1}$ AND NIV DRORY ${ }^{2}$ \\ ${ }^{1}$ Department of Astronomy, The University of Texas at Austin, 1 University Station C1400, Austin, TX 78712, USA; dbfisher@astro.as.utexas.edu \\ ${ }^{2}$ Max-Planck-Institut für Extraterrestrische Physik, Giessenbachstraße, 85748 Garching, Germany; drory@ mpe.mpg.de \\ Received 2007 September 27; accepted 2008 April 21; published 2008 July 14
}

\begin{abstract}
In this paper, we study the properties of pseudobulges (bulges that appear similar to disk galaxies) and classical bulges (bulges which appear similar to E-type galaxies) in bulge-disk decompositions. We show that the distribution of bulge Sérsic indices, $n_{b}$, is bimodal, and this bimodality correlates with the morphology of the bulge. Pseudobulges have $n_{b} \lesssim 2$ and classical bulges have $n_{b} \gtrsim 2$ with little to no overlap. Also, pseudobulges do not follow the correlations of Sérsic index with structural parameters or the photometric projections of the fundamental plane in the same way that classical bulges and elliptical galaxies do. We find that pseudobulges are systematically flatter than classical bulges and thus more disk-like in both their morphology and shape. We do not find significant differences between different bulge morphologies which we are collectively calling pseudobulges (nuclear spirals, nuclear rings, nuclear bars, and nuclear patchiness); they appear to behave similarly in all parameter correlations. In the Sérsic index, flattening, and bulge-to-total ratio, the distinction appears to be between classical bulges and pseudobulges, not between different pseudobulge morphologies. The Sérsic index of the pseudobulges does not correlate with $B / T$, in contrast to classical bulges. Also, the half-light radius of the pseudobulge correlates with the scale length of the disk; this is not the case for classical bulges. The correlation of Sérsic index and scale lengths with bulge morphology suggests that secular evolution is creating pseudobulges with low-Sérsic index and that other processes (e.g., major mergers) are responsible for the higher Sérsic index in classical bulges and elliptical galaxies.
\end{abstract}

Key words: galaxies: bulges - galaxies: formation - galaxies: fundamental parameters - galaxies: structure

\section{INTRODUCTION}

Historically, all bulges were assumed to be little elliptical galaxies residing in the centers of galactic disks. Thus, it was also assumed that all bulges were dynamically hot stellar systems. Recent work puts this assumption in question. Many bulges have disk-like features that do not resemble E-type galaxies.

Classical bulges are dynamically hot, and relatively featureless; they appear similar to the end products of galaxy major mergers. They are easily recognized as having morphologies very similar to E-type galaxies.

In contrast, many bulges have features that are not found in elliptical galaxies, but in galactic disks. These features include the following: kinematics dominated by rotation (Kormendy 1993), flattening similar to that of their outer disk (Fathi \& Peletier 2003; Kormendy 1993), mid-IR colors of the bulge similar to those of the outer disk (Fisher 2006), nuclear bar (Erwin $\&$ Sparke 2002), nuclear ring and/or nuclear spiral (Carollo et al. 1997), and near-exponential surface brightness profiles (Andredakis \& Sanders 1994). Bulges with such properties are called pseudobulges. All of these phenomena are manifestations of stellar systems that are dynamically cold. However, the extent to which these features exist simultaneously in all pseudobulges is not yet well understood. For a review of the properties of pseudobulges see Kormendy \& Kennicutt (2004) (KK04 hereafter).

In this paper, we refer to the super-set of the two systems simply as bulges. We note that in this paper the term pseudobulge refers to bulges with morphology reminiscent of disk galaxies; there is no a priori assumption about their formation mechanism.

Investigating the link between bulge morphology and bulge structural parameters is the main interest of this paper. More precisely, those bulges with morphologies that are reminiscent of disks (such as nuclear rings, nuclear bars, and nuclear spirals) are expected to have lower Sérsic index than those bulges with smooth light distributions resembling E-type galaxies. We will investigate whether this expectation holds and whether the distribution of bulge Sérsic indices is dichotomous.

Determining whether a critical Sérsic index that discriminates between classical bulges and pseudobulges exists, and what its value is, impacts our understanding of bulge formation in at least two ways. First, the distribution of Sérsic indices would constrain formation theories of classical bulges and pseudobulges. Also, the existence of a critical Sérsic index would robustly establish a method for distinguishing pseudobulges from classical bulges without using high-resolution imaging or kinematic data, neither of which is currently available in large surveys.

In the last ten years, the subject of surface brightness profiles of bulges and elliptical galaxies has experienced a shift of paradigm. Traditionally, surface brightness profiles of elliptical galaxies and bulges were all thought to be of a single shape that is well characterized by the de Vaucauleurs $r^{1 / 4}$ profile. Caon et al. (1994) show that surface brightness profiles of elliptical galaxies are better fit by Sérsic profiles (Sérsic 1968), which generalize the exponent in the de Vaucauleurs profile to a free parameter. Also, Andredakis \& Sanders (1994) show that many bulge-disk galaxies are better described by a double exponential than an inner $r^{1 / 4}$ profile with an outer exponential. Andredakis et al. (1995) generalize this to show that bulge-disk galaxies are better fit by inner Sérsic profiles with outer exponential disks than double exponentials profiles. The Sérsic function plus outer disk model for bulge-disk galaxies reads

$$
I(r)=I_{0} \exp \left[-\left(r / r_{0}\right)^{1 / n_{b}}\right]+I_{d} \exp [-(r / h)],
$$

where $I_{0}$ and $r_{0}$ represent the central surface brightness and scale length of the bulge, $I_{d}$ and $h$ represent the central surface 
brightness and scale length of the outer disk, and $n_{b}$ represents the bulge Sérsic index.

It is not surprising that Sérsic profiles fit bulge surface brightness profiles better than $r^{1 / 4}$ profiles, since the Sérsic function has more flexibility due to the extra parameter. However, the new parameter, $n_{b}$, correlates with many properties of the stellar systems to which it is fitted, including but not limited to the following: velocity dispersion $\sigma$ (Khosroshahi et al. 2000), absolute magnitude (Graham et al. 1996), and effective radius (Caon et al. 1994). Many authors have shown that these correlations extend to bulges of bulge-disk galaxies (Graham 2001; MacArthur et al. 2003; de Jong et al. 2004; Thomas \& Davies 2006). Additionally, Andredakis et al. (1995) show that the Sérsic index of bulges correlates with Hubble type, decreasing from $n_{b} \sim 3.7$ for S0 galaxies to $n_{b} \sim 1.6$ in Sbc-Sd galaxies. Therefore, it is reasonable to assume that it has physical significance. The Sérsic index is often referred to as the shape parameter, as it is generally taken as a surrogate for properties such as concentration of the surface brightness profile. For a review of the properties of Sérsic profiles, see Graham \& Driver (2005).

The tentative assumption is that the Sérsic index of a bulge reflects the classical bulge-pseudobulge dichotomy. Lower Sérsic index might indicate that a bulge is more likely to be a pseudobulge. We do not understand the mechanism that is responsible for determining the Sérsic indices in pseudobulges (or classical bulges). Yet, it seems plausible that the light distribution is similar to that of a disk, since so many other properties of pseudobulges are similar to those of galactic disks. Courteau et al. (1996) use bulge-disk decompositions of 243 galaxies to show that the $85 \%$ of bulges in $\mathrm{Sb}-\mathrm{Sc}$ galaxies are better fitted by the double exponential than cuspier $r^{1 / 4}$ models. Thus, the common conclusion is that pseudobulges are marked by near-exponential Sérsic index (KK04). Scarlata et al. (2004) show with Space Telescope Imaging Spectrograph (STIS) acquisition images that bulges with surface brightness profiles more resembling exponential profiles are more likely to have disk-like morphology (e.g., spiral arms), yet there is significant scatter to this claim, in their sample. They go on to show that the distribution of central slopes of surface brightness profiles of the bulges in their sample is bimodal when plotted against absolute magnitude.

It is yet unknown where pseudobulges should lie in other parameter correlations, such as fundamental plane projections. We do not expect that pseudobulges occupy a significantly different location than classical bulges in fundamental plane parameter space, since the fundamental plane is not known to be bimodal. Many studies of the locations of bulges in structural parameter space exist (e.g., Bender et al. 1992; Graham 2001; MacArthur et al. 2003; de Jong et al. 2004; Thomas \& Davies 2006), and no significant bimodal behavior is noticed. Carollo (1999) remarks, though, that pseudobulges deviate more from the $\mu_{e}-r_{e}$ relation (Kormendy 1977). We will investigate this further in this paper. Kormendy (1993) shows that the majority of bulges are rounder than the outer parts of the disks they reside in, yet a significant minority are as flat as their associated outer disk. A few bulges are even flatter than their outer disk. This behavior correlates with Hubble type; bulges in later-type galaxies have flattening more similar to that of the associated outer disk. Fathi $\&$ Peletier (2003) carried out bulge-disk decompositions of 70 galaxies on higher-resolution data finding a similar result.

As discussed in KK04, pseudobulges are characterized principally by having less random motion per unit stellar light. They are rotation-dominated systems (Kormendy 1993; Kormendy \& Kennicutt 2004). Thus it makes sense that they be flatter. The relative flatness of a bulge to its associated outer disk has also been suggested as a pseudobulge indicator (KK04). We will test this hypothesis in this paper by comparing the flatness of bulges with disk like morphologies to that of bulges with morphologies like those of elliptical galaxies.

The properties of pseudobulges are in stark contrast to the expected end result of the hierarchical merging process; one does not expect violent relaxation to produce spiral structure and dynamics that is dominated by ordered motion. Further scenarios for the formation of bulges have been suggested. Clump instabilities in disks at high redshift can form bulge-like structures in simulations (Noguchi 1999). It is also plausible that gas-rich accretion could form dynamically cold bulges. Internal evolution of disks can drive gas and stars to the center of a disk galaxy as well. The population of bulges as a whole and any one particular bulge may be the result of more than one of these processes.

However, the connections between bulge and disk stellar populations (Peletier \& Balcells 1996; MacArthur et al. 2004), interstellar medium (ISM) (Regan et al. 2001; Fisher 2006) and scale lengths (Courteau et al. 1996) may suggest that pseudobulges form through processes intimately linked to their host disks. Furthermore, Drory \& Fisher (2007) find that classical bulges occur in red-sequence galaxies and pseudobulges occur in blue cloud galaxies. Kormendy \& Kennicutt (2004) review the case that pseudobulges are not the result of major mergers, but rather that internal disk evolution may be responsible for them. However, the fact that bulges can form out of the internal evolution of a disk is not a new idea, see, for example, Hohl (1975).

Simulations suggest that if disk galaxies do not experience major mergers, they may evolve by redistribution of energy and angular momentum driven by non-axisymmetries such as bars, ovals, and spiral structure (Simkin et al. 1980; Pfenniger \& Friedli 1991; Debattista et al. 2004), resulting in star formation and bulge-like stellar densities, thus forming pseudobulges. Indeed, a correlation between central star-formation rate and the presence of bars and ovals has been detected (e.g., Sheth et al. 2005; Jogee et al. 2005; Fisher 2006). Also, Peeples \& Martini (2006) find that galaxies with nuclear rings and/or nuclear spirals are more strongly barred. Thus, we also investigate the possible connection between driving mechanisms and structural properties.

It is, thus, possible that the absence of a classical bulge in a galaxy indicates that the galaxy has not experienced a major merger since the formation of the disk. In this context, pseudobulges may be thought of as more similar to pure disk galaxies that have a surface brightness profile which breaks from the outer exponential profile to a more steep inner surface brightness profile. Though the frequency of pseudobulges has not yet been robustly calculated, if they are common then this implies that many disk galaxies did not suffer major mergers since their formation.

This paper is organized as follows. In Section 2, we present the observational data we use and we discuss the surface brightness fitting procedure. In Section 3, we present results on the location of pseudobulges and classical bulges in various structural parameter correlations. In Section 4, we discuss the flatness of pseudobulges and classical bulges. In Section 5, we discuss behavior of different bulge morphologies (nuclear bars, nuclear spirals, and nuclear rings) in various parameter 


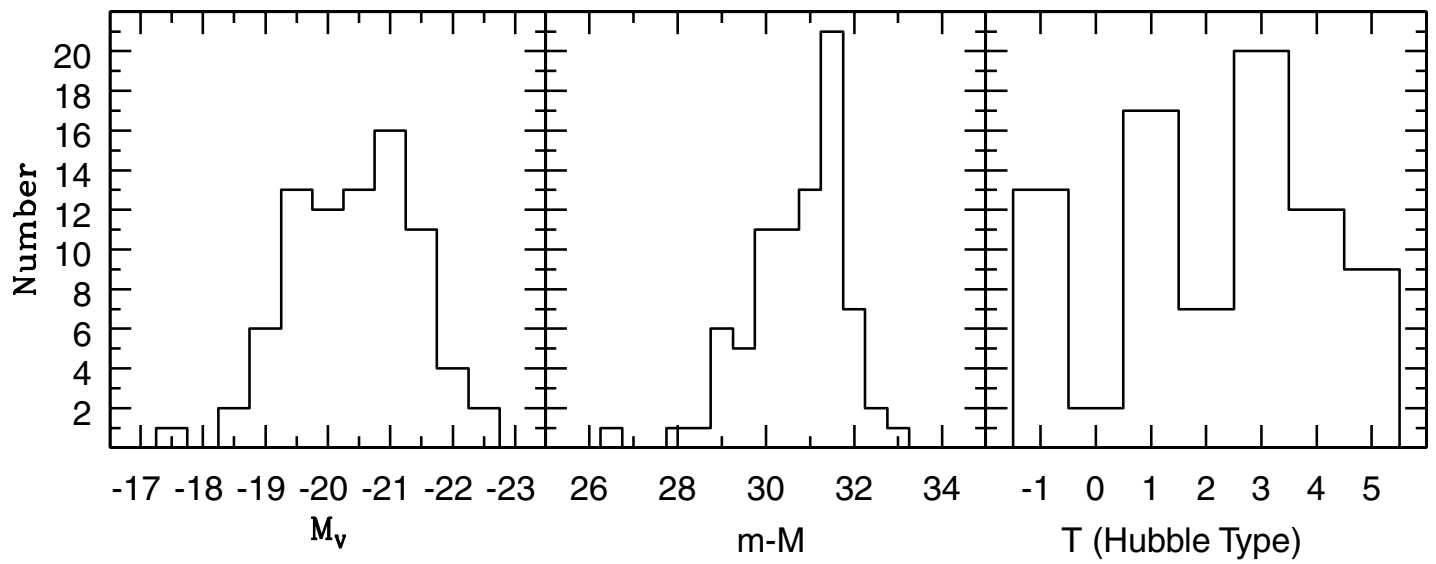

Figure 1. Distribution of absolute magnitude (left), distance modulus (middle), and Hubble type (right) for all 77 galaxies in our sample.

correlations. In Section 6, we summarize and discuss these results. Finally, the appendix includes an image of each galaxy, all decompositions and a discussion of the robustness of our decomposition and fitting procedure.

\section{METHODS AND OBSERVATIONS}

\subsection{The Sample}

The aim of this work is to establish whether or not pseudobulges-recognized by the presence of disk-like morphological features as motivated and discussed in KK04-can be distinguished from classical bulges simply by structural features in their surface brightness profiles, most prominently their profile shape. Thus, we ask whether bulges that contain disklike morphologies (pseudobulges) have lower Sérsic index and higher flattening ratios than bulges with elliptical-like morphologies (classical bulges). Answering this question requires high-resolution imaging (preferably in the optical bands) to detect the nuclear spirals, bars, and rings; and we need surface brightness profiles with large dynamic range in radius to accurately determine the parameters in Equation (1) for a bulge-disk decomposition.

We choose galaxies observable from the northern hemisphere that have data in the Hubble Space Telescope (HST) archive. We limit our selection to galaxies closer than $\sim 40 \mathrm{Mpc}$ to resolve features in the bulge.

The link between non-axisymmetries (barred and oval distortions) and secular evolution motivates us to create a sample containing roughly equal numbers of galaxies with a driving agent (galaxies with a bar and/or an oval) and galaxies without a driving agent. Detection of oval distortions is discussed in Kormendy (1982). They are identified by nested shelves in the surface brightness profile usually having different position angles. We identify bars by consulting the Carnegie Atlas of Galaxies (Sandage \& Bedke 1994) and the RC3 (de Vaucouleurs et al. 1991). If a galaxy has both a bar and an oval, we call that galaxy barred. Additionally, we look for bars and ovals in all galaxies using $K$-band images from Two Micron All Sky Survey (2MASS) (Skrutskie et al. 2006). Note that we do not distinguish grand design spirals as a possible secular driver, though they may be able to generate a similar but less extreme effect as bars do (KK04). We use 39 undriven (no bar and no oval) galaxies and 38 driven galaxies ( 30 barred and 8 ovaled), a total of 77 galaxies.

In Figure 1, we show the distribution of global properties of the galaxies in our sample; these are also listed in Table 1 . The distribution of the distances of the galaxies in our sample is heavily peaked at $16 \mathrm{Mpc}$ due to the Virgo cluster and has a standard deviation of $6 \mathrm{Mpc}$. We derive total magnitudes by 2D integration of our surface brightness profiles. The distribution of absolute $V$ magnitudes ranges mostly from -19 to -22 with a median value of -20.5 .

KK04 compiles data from several different studies to generate preliminary statistics on how the frequency of pseudobulges varies along the Hubble Sequence. They suggest that both pseudobulges and classical bulges exist at intermediate Hubble types ( $\mathrm{S} 0$ to $\mathrm{Sbc}$ ). There appears to be a transition from classical bulges being more frequent at early types $(\mathrm{S} 0-\mathrm{Sb})$ to pseudobulges being more frequent at later types (Sbc-Sd). They further suggest that classical bulges will be almost nonexistent at Hubble types Sc and later. In the right panel of Figure 1, we show the distribution of Hubble types (taken from Sandage \& Bedke 1994) of the galaxies in our sample. To test for differences between pseudobulges and classical bulges we choose to sample the range of Hubble type from S0 to Sc. If we combine this with our choice of evenly sampling driven and undriven galaxies, we expect that our sample should overemphasize pseudobulges. The distribution of Hubble types is as follows: 13 S0, 2 S0a, $18 \mathrm{Sa}, 6 \mathrm{Sab}, 20 \mathrm{Sb}, 11 \mathrm{Sbc}$, and $9 \mathrm{Sc}$. We will also use 24 E-type galaxies from Kormendy et al. (2008) as early-type sample in some parameter correlations.

\subsection{Identification of Pseudobulges}

We define "bulges" photometrically as excess light over the inward extrapolation of the surface brightness profile of the outer disk. The region of the galaxy where this excess light dominates the profile is the bulge region. We classify galaxies as having a pseudobulge by their morphology within this bulge region; if the bulge is or contains any of the following features: a nuclear bar, a nuclear spiral, and/or a nuclear ring, then the bulge is called a pseudobulge. Conversely, if the bulge better resembles an elliptical galaxy (relatively featureless isophotes), then the bulge is called a classical bulge. This method is discussed in KK04. The existence/absence of visibly identifiable disk-like structure in a bulge correlates with the properties of the bulge and the whole galaxy. The same method is shown to be successful in identifying bulges with higher specific star-formation rates (Fisher 2006) and globally bluer galaxies (Drory \& Fisher 2007).

Figure 2 shows high-resolution $H S T$ images of the bulge region of all galaxies in our sample. All images are taken in close 
Table 1

Data Sources

\begin{tabular}{|c|c|c|c|c|c|}
\hline No. & Instrument & Filter & $\begin{array}{c}\text { Scale } \\
\left(\operatorname{arcsec} \text { pixel }^{-1}\right)\end{array}$ & $\begin{array}{l}\text { Field of view } \\
\quad(\operatorname{arcmin})\end{array}$ & Reference $^{\mathrm{a}}$ \\
\hline 1 & CFHT HRCam & $\mathrm{V}$ & 0.11 & $1.9 \times 1.2$ & Kormendy (2008) \\
\hline 2 & CTIO $1.5 \mathrm{~m}$ & $\mathrm{~V}$ & 0.434 & $14.8 \times 14.8$ & Kennicutt et al. (2003) (NED) \\
\hline 3 & CTIO 4.0 Mosaic 2 & $\mathrm{~V}$ & 0.27 & $9.3 \times 9.3$ & Kennicutt et al. (2003) (NED) \\
\hline 4 & Danish 1.54 DFOSC & $\mathrm{V}$ & 0.4 & $13.7 \times 13.7$ & Larsen \& Richtler (1999) (NED) \\
\hline 5 & HST ACS/WFC & F547M, F555W, F606W & 0.049 & $3.5 \times 3.4$ & MAST \\
\hline 6 & $H S T$ WFPC1 (PC) & F555W & 0.043 & $1.1 \times 1.1$ & Lauer et al. (1995) \\
\hline 7 & HST WFPC 2 (PC) & F547M, F555W, F606W & 0.0455 & $0.6 \times 0.6$ & MAST, Lauer et al. (2005) \\
\hline 8 & $H S T$ WFPC 2 (WF) & F547M, F555W, F606W & 0.0996 & $1.3 \times 1.3$ & MAST \\
\hline 9 & ING JKT AGBX & $\mathrm{V}$ & 0.3 & $7.0 \times 1.9$ & de Jong (1996) (NED) \\
\hline 10 & ING JKT SITe2 & $\mathrm{V}$ & 0.33 & $11.2 \times 11.2$ & ING, Knapen et al. (2004) (NED) \\
\hline 11 & ING INT WFC & $\mathrm{V}$ & 0.33 & $34 \times 34$ & ING \\
\hline 12 & KPNO $2.1 \mathrm{~m} \mathrm{~T} 2 \mathrm{KA}$ & $\mathrm{V}$ & 0.304 & $10.4 \times 10.4$ & Kennicutt et al. (2003) (NED) \\
\hline 13 & KPNO 0.9 m/TEK5 & $\mathrm{V}$ & 0.78 & $9.2 \times 7.0$ & Kennicutt et al. (2003) (NED) \\
\hline 14 & LCO $2.5 \mathrm{~m}$ & $\mathrm{~V}$ & 0.26 & $8.8 \times 8.8$ & Kuchinski et al. (2000) (NED) \\
\hline 15 & McDonald $0.8 \mathrm{~m}$ PFC & $\mathrm{V}$ & 1.36 & $46.2 \times 46.2$ & New observation \\
\hline 16 & MLO 40 in & $\mathrm{V}$ & 0.985 & $13.0 \times 13.0$ & Cheng et al. (1997) (NED) \\
\hline 17 & NOT ALFOSC & $\mathrm{V}$ & 0.189 & $6.5 \times 6.5$ & Larsen \& Richtler (1999) (NED) \\
\hline 18 & SDSS & $\mathrm{g}, \mathrm{r}$ & 0.396 & $13.5 \times 9.8$ & Adelman-McCarthy et al. (2006) \\
\hline 19 & Palomar $1.5 \mathrm{~m}$ WideField & $\mathrm{V} ; \mathrm{g}, \mathrm{r}$ & 1.19 & $14.3 \times 14.3$ & Kuchinski et al. (2000) (NED), Frei et al. (1996) (NED) \\
\hline 20 & WHT Cass TEK1 & $\mathrm{V}$ & 0.241 & $4.1 \times 4.1$ & ING \\
\hline
\end{tabular}

Notes.

a Most commonly, the publication of the data is referenced. If it is found on NED this is noted. "MAST" refers to the Multimission Archive at STScI. "ING" refers to the Archive of ING telescope data. 


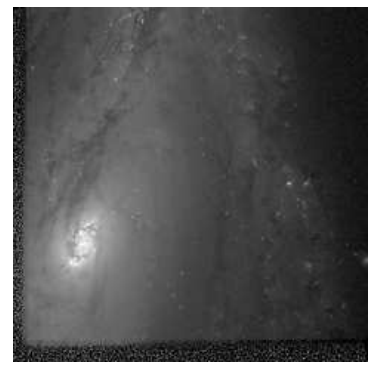

N613 WF2 F606W p

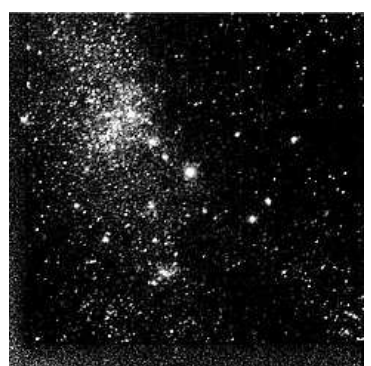

N1313 PC2 F606W p

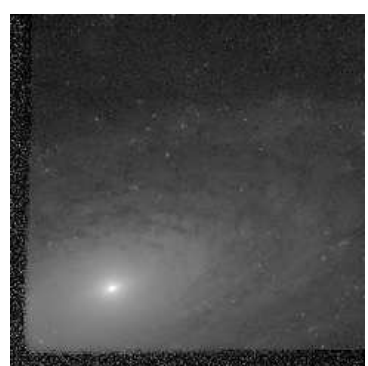

N1425 WF2 F606W c

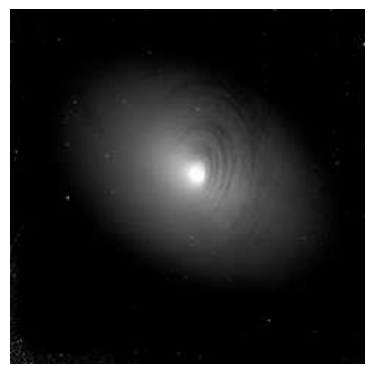

N2787 PC2 F555W p

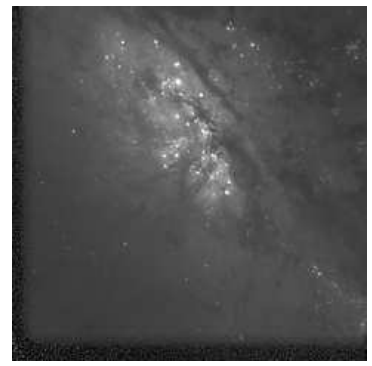

N2903 PC2 F606W p

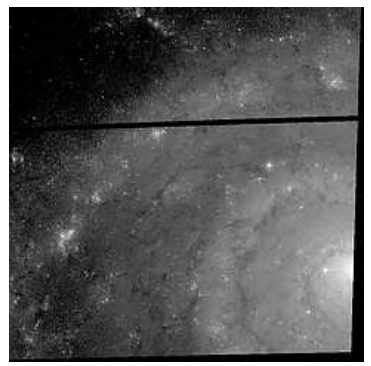

N628 ACS F555W p

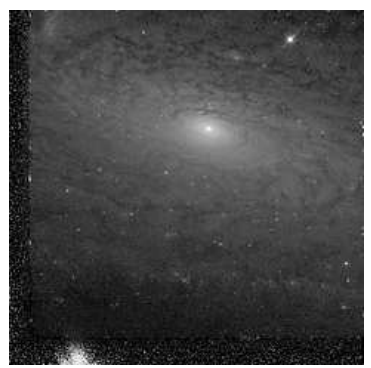

N1325 PC2 F555W p

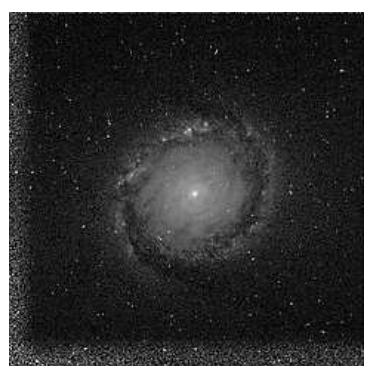

N1512 PC2 F547M p

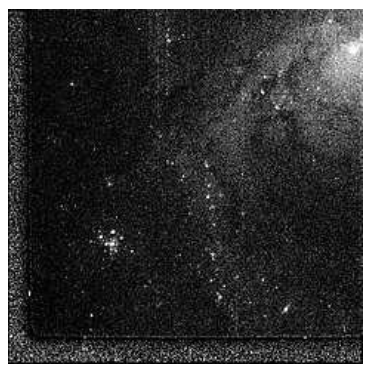

N2835 PC2 F606W p

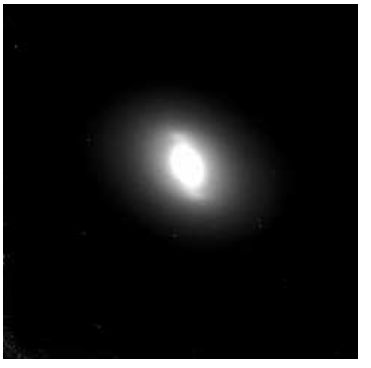

N2950 PC2 F555W p

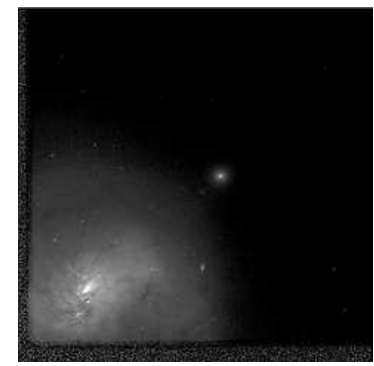

N1022 WF2 F606W p

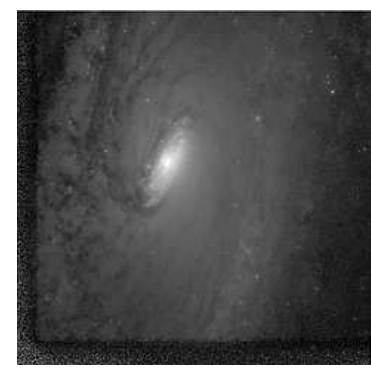

N1353 PC2 F606W p

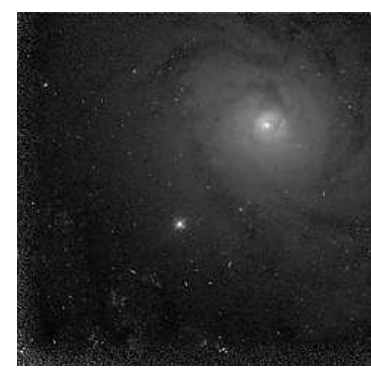

N1566 PC2 F555W p

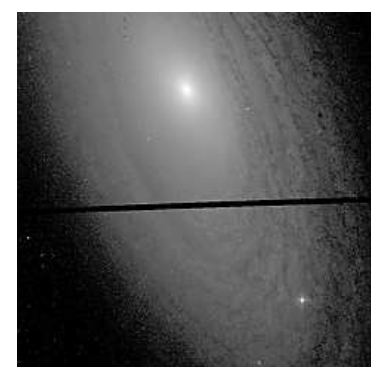

N2841 ACS F814W

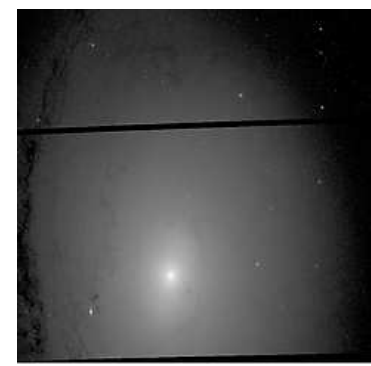

N3031 ACS F606W c

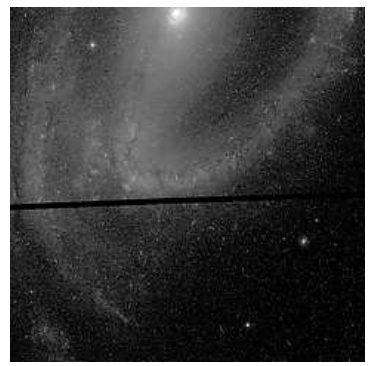

N1300 ACS F555W p

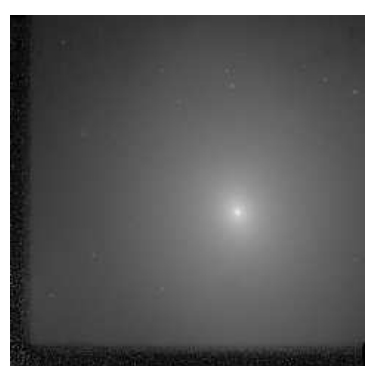

N1398 PC2 F606W c

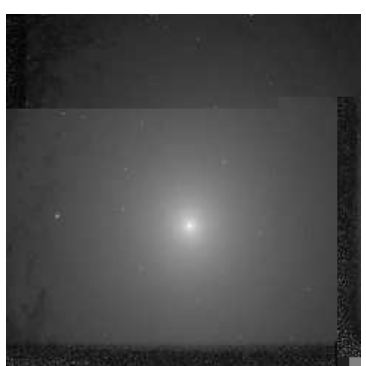

N2775 PC2 F606W c

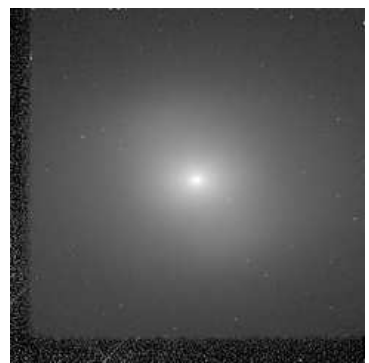

N2880 PC2 F555W c

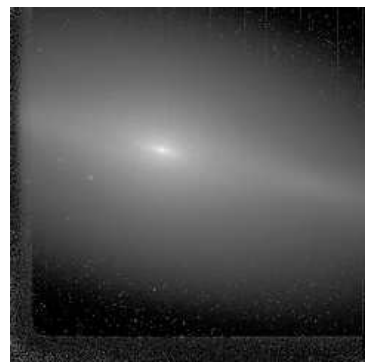

N3115 PC2 F555W c

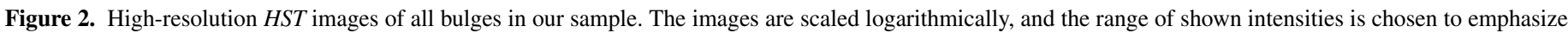

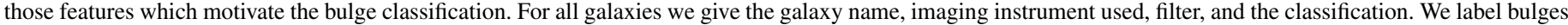
classified as classical by "c" and pseudobulges by "p." The images show the inner 40 arcsec (ACS), 36 arcsec (PC 2), and 80 arcsec (WF2) of the galaxies. 


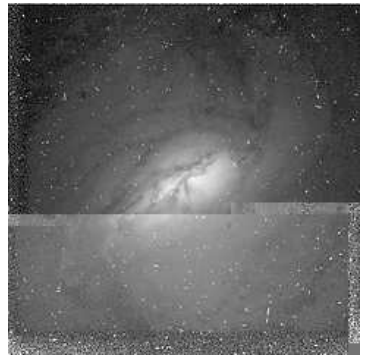

N3166 PC2 F547M p

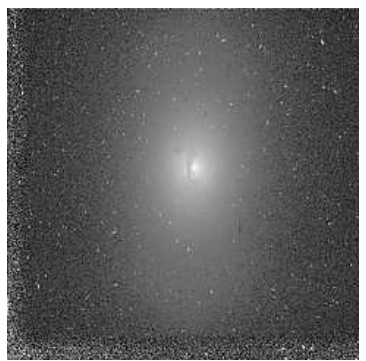

N3245 PC2 F547M c

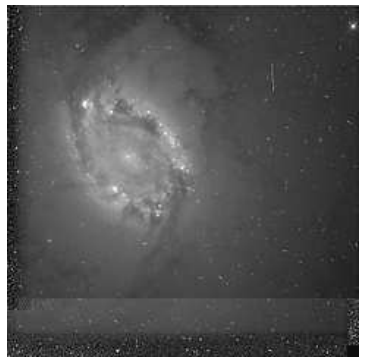

N3351 PC2 F606W p

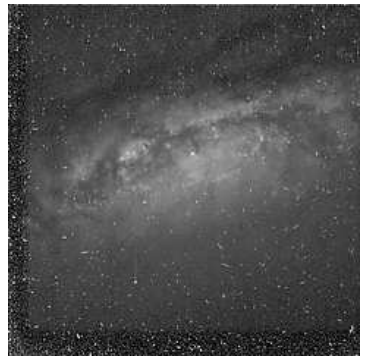

N3593 PC2 F547M p

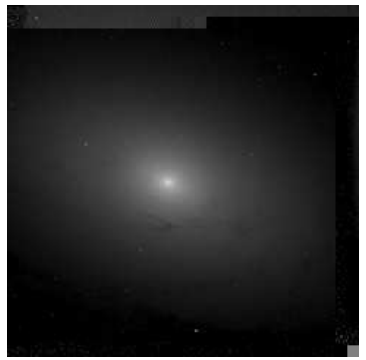

N3898 PC2 F606W c

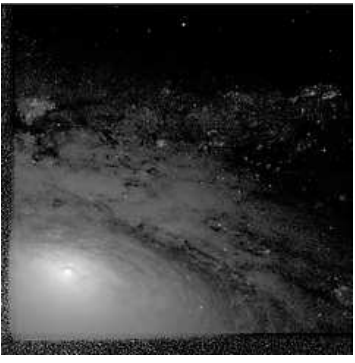

N3169 WF2 F606W p

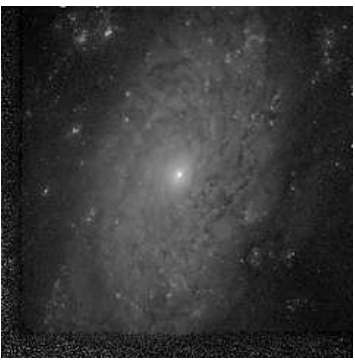

N3259 PC2 F606W p

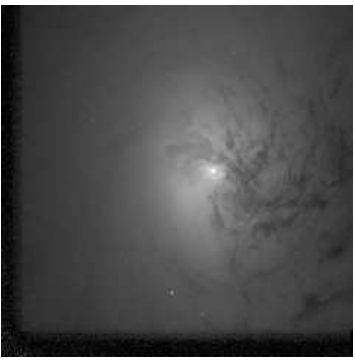

N3368 PC2 F606W p

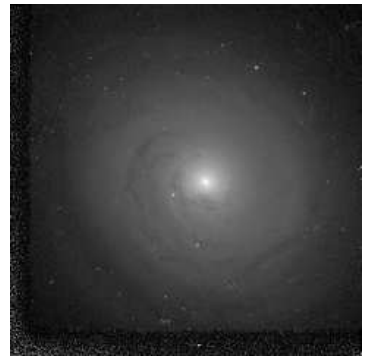

N3277 PC2 F606W c

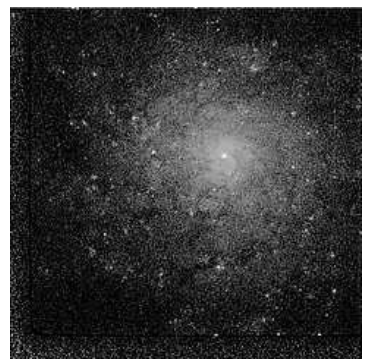

N3423 PC2 F606W p

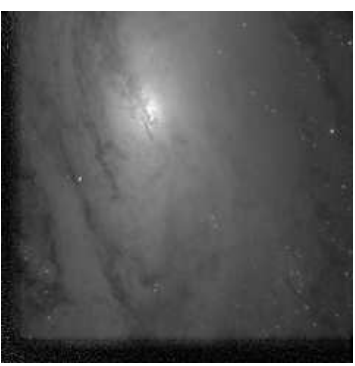

N3627 PC2 F606W p

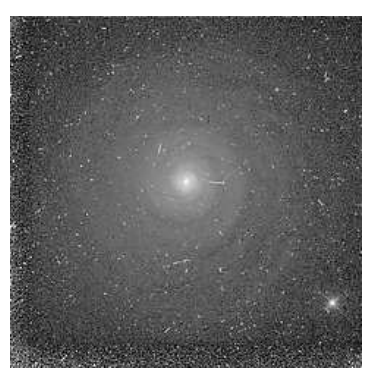

N3642 PC2 F547M p

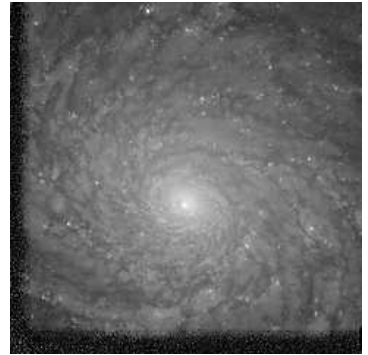

N4030 PC2 F606W p
N3885 WFPC2 F606W $\mathrm{p}$

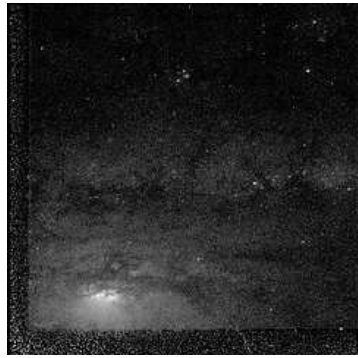

N3198 PC2 F606W p

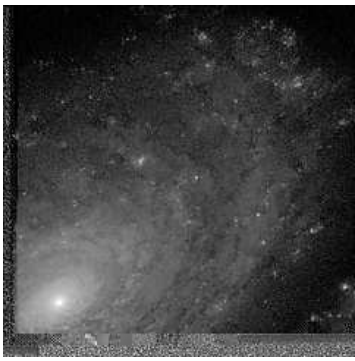

N3338 WF2 F606W p

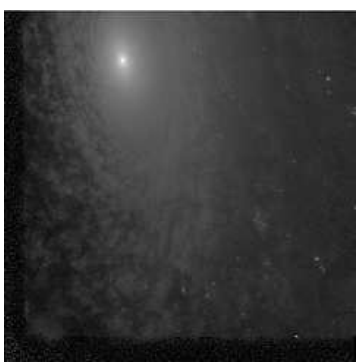

N3521 PC2 F606W c
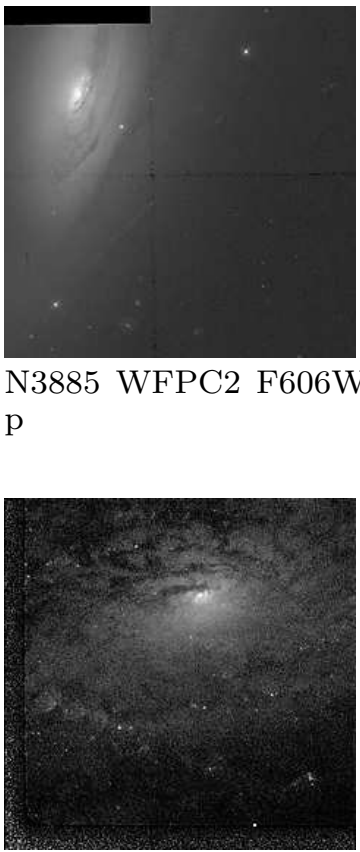

N4062 PC2 F606W p

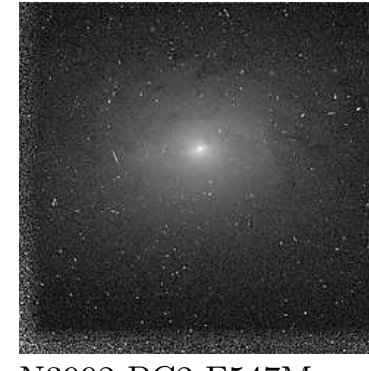

Figure 2. (Continued) 


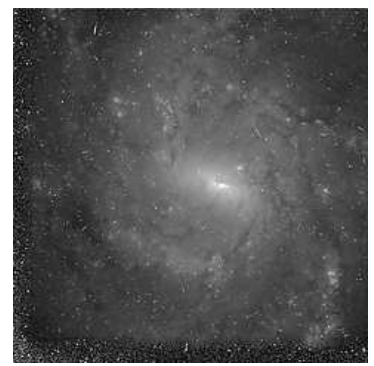

N4152 PC2 F606W p

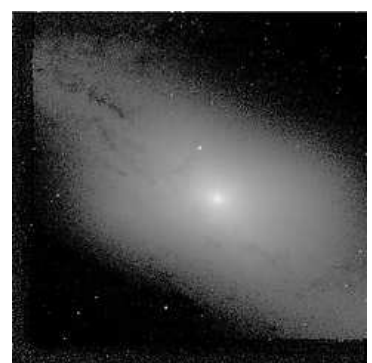

N4260 PC2 F606W c

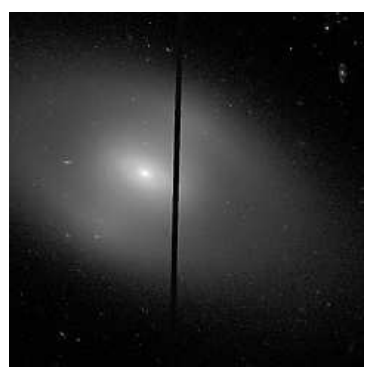

N4371 ACS F475W p

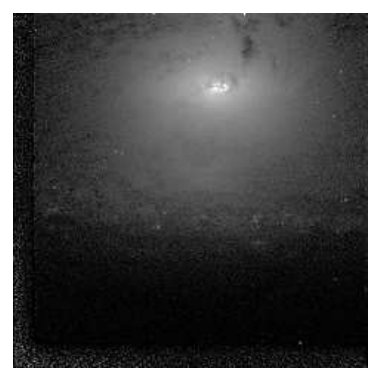

N4448 PC2 F606W p

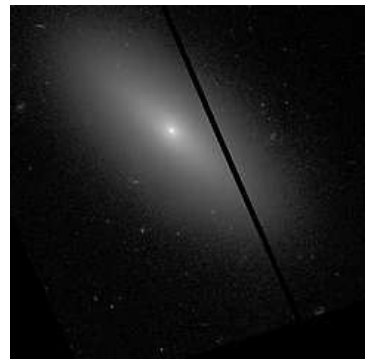

N4564 ACS F475W c

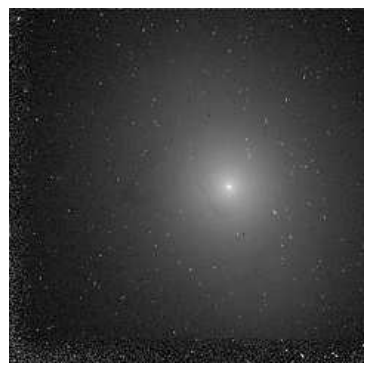

N4203 PC2 F555W c

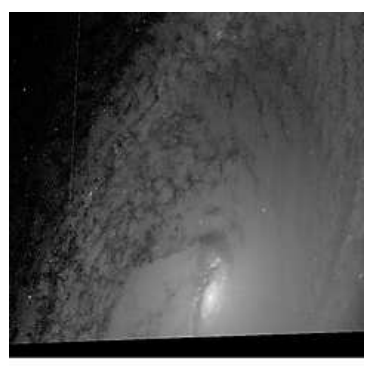

N4274 ACS F606W p

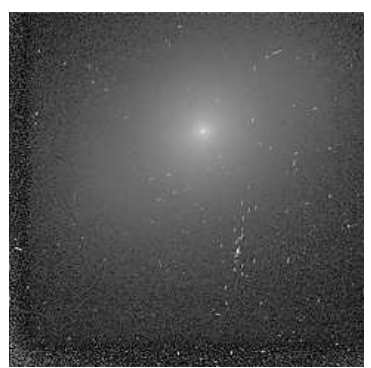

N4379 PC2 F555W c

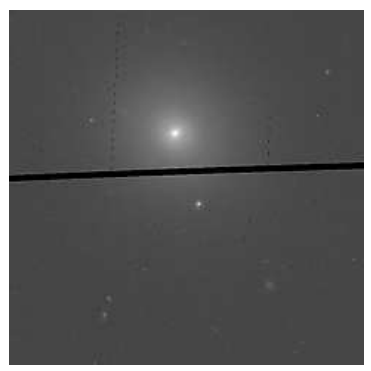

N4489 ACS F475W c

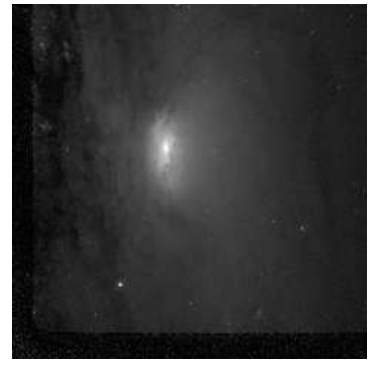

N4569 PC2 F555W p

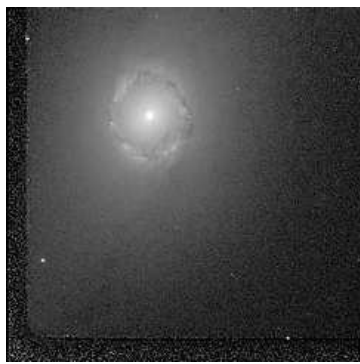

N4245 PC2 F606W p

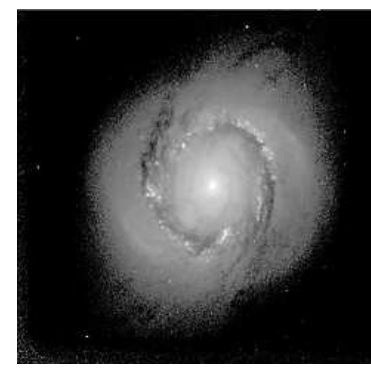

N4314 PC2 F606W p

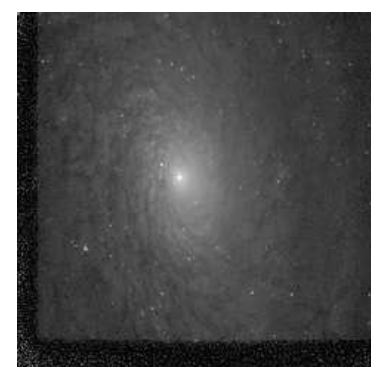

N4380 PC2 F606W p

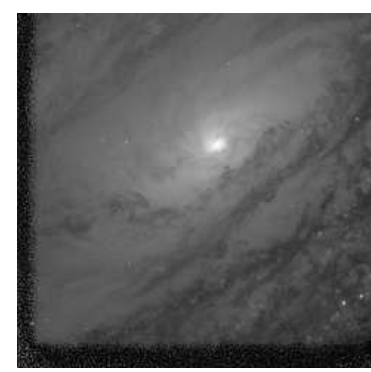

N4501 PC2 F606W p

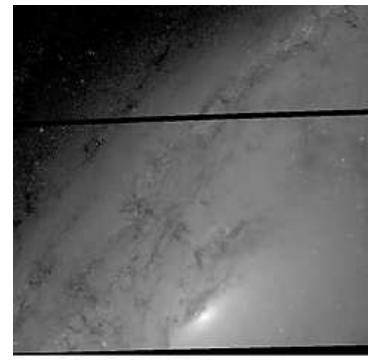

N4258 ACS F606W p

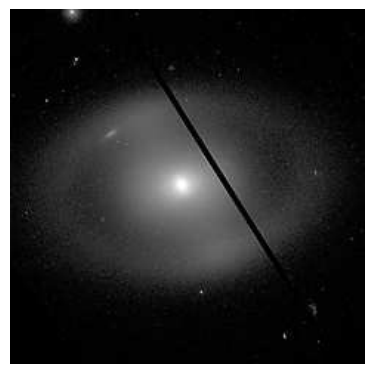

N4340 ACS F475W p

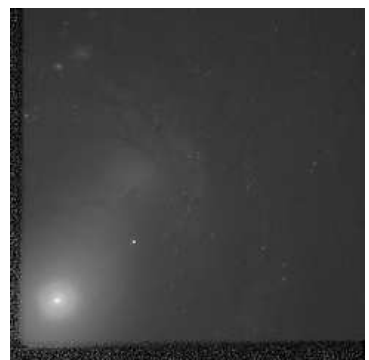

N4394 WF2 F606W p

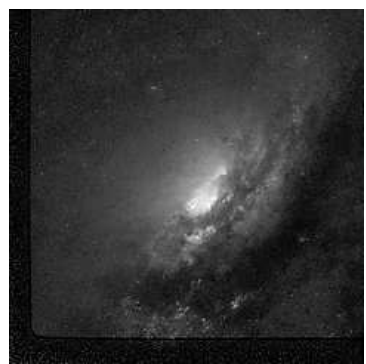

N4536 PC2 F555W p

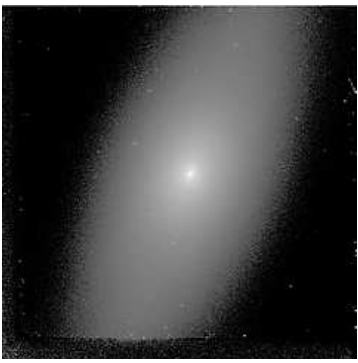

N4570 PC2 F555W c

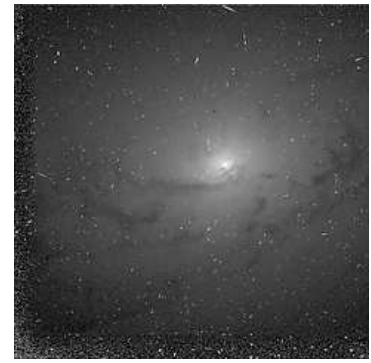

N4579 PC2 F547M p

Figure 2. (Continued) 


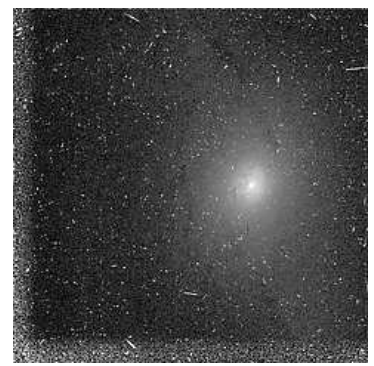

N4639 PC2 F547M p

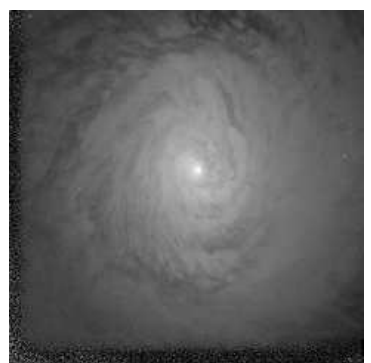

N4736 PC2 F555W p

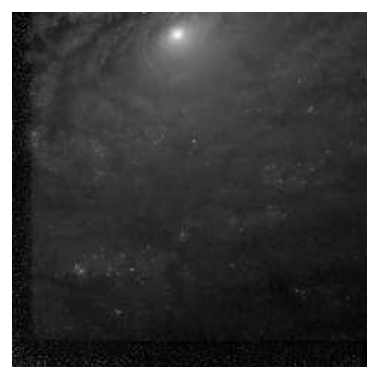

N5055 PC2 F606W p

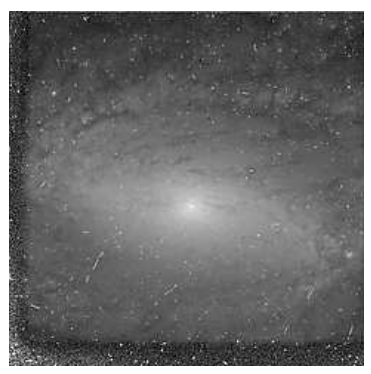

N5970 PC2 F606W p

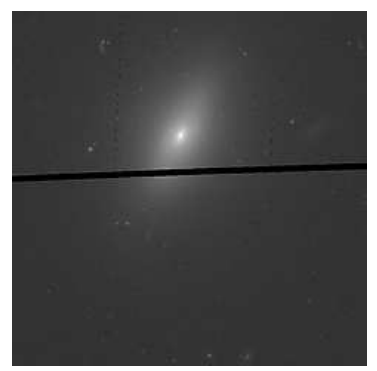

N4660 ACS F475W c

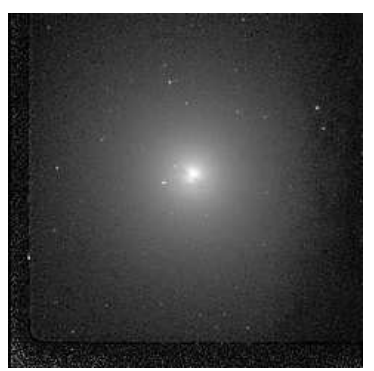

N4772 PC2 F606W c

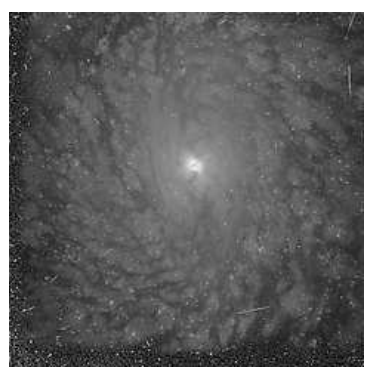

N5194 PC2 F547M p

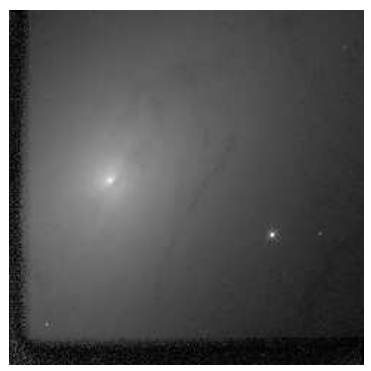

N6744 PC2 F606W c

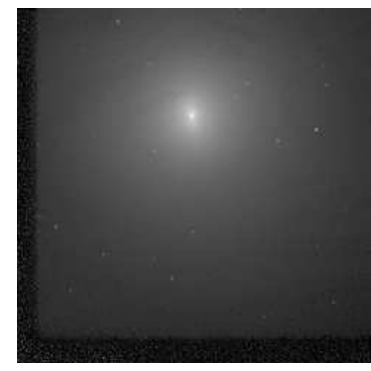

N4698 PC2 F606W c

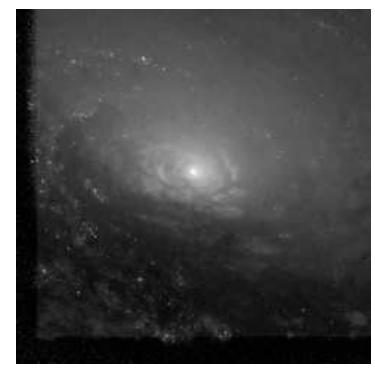

N4826 PC2 F547M p

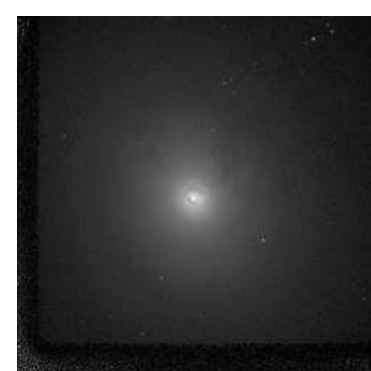

N5273 PC2 F606W c

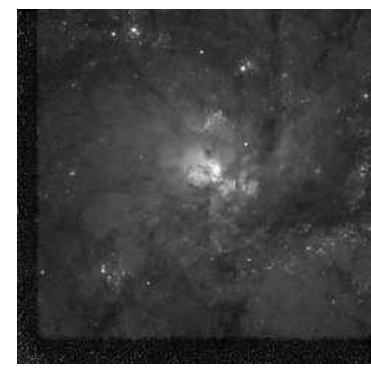

N6946 PC2 F606W p

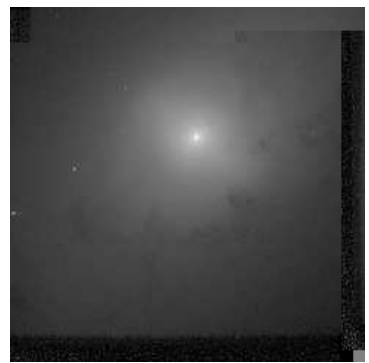

N4725 PC2 F606W c

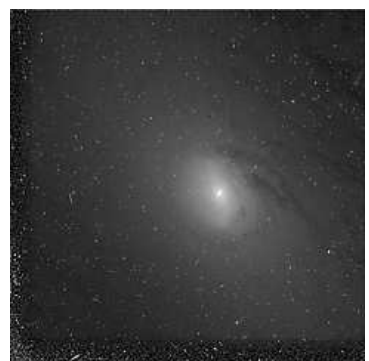

N4941 PC2 F606W p

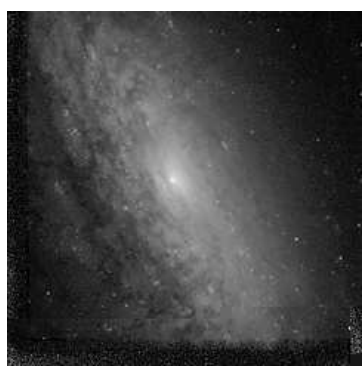

N5879 PC2 F606W p

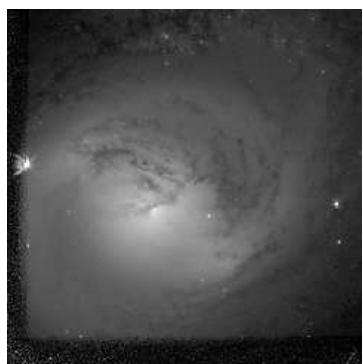

N7177 PC2 F606W p

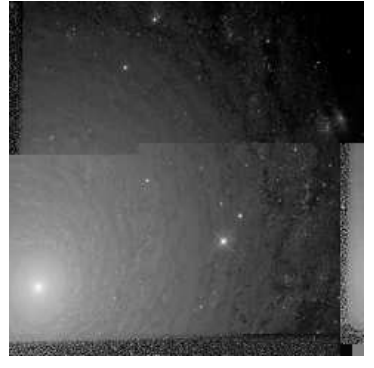

N7217 WF2 F606W p

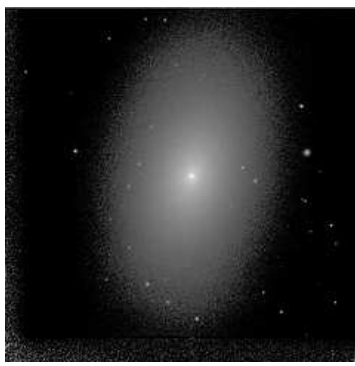

N7457 PC2 F555W c

Figure 2. (Continued)

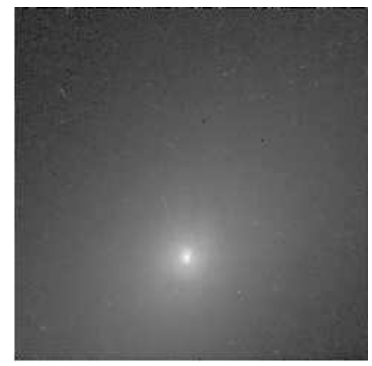

N7743 PC2 F606W c 
Table 2

Sample Galaxy Properties

\begin{tabular}{|c|c|c|c|c|c|c|c|}
\hline Identifier & $\begin{array}{c}\text { Hubble }^{\mathrm{a}} \\
\text { type }\end{array}$ & $\begin{array}{c}\text { Driving } \\
\text { mechanism }\end{array}$ & $\begin{array}{c}a_{V} \mathrm{~b} \\
(\mathrm{mag})\end{array}$ & $\begin{array}{c}V \\
(\mathrm{mag})\end{array}$ & $\begin{array}{c}M_{V} \\
(\mathrm{mag})\end{array}$ & $\begin{array}{c}\text { Distance }^{c} \\
(\mathrm{Mpc})\end{array}$ & Data sources $^{\mathrm{d}}$ \\
\hline NGC 613 & $\mathrm{SBb}(\mathrm{rs})$ & Bar & 0.06 & 10.28 & -21.31 & 20.13 & 8,15 \\
\hline NGC 628 & $\mathrm{Sc}(\mathrm{s})$ & $\ldots$ & 0.23 & 9.24 & -20.77 & 9.04 & $2,5,10,17$ \\
\hline NGC 1022 & $\mathrm{SBa}(\mathrm{r})$ & Bar & 0.09 & 11.32 & -19.74 & 15.63 & 8,15 \\
\hline NGC 1300 & $\mathrm{SBb}(\mathrm{s})$ & Bar & 0.10 & 10.57 & -21.05 & 20.15 & $5,10,15$ \\
\hline NGC 1313 & $\mathrm{SBc}(\mathrm{s})$ & Bar & 0.36 & 9.23 & -20.41 & 7.15 & $4,7,14$ \\
\hline NGC 1325 & $\mathrm{Sb}(\mathrm{s})$ & $\ldots$ & 0.07 & 11.63 & -19.97 & 20.20 & 7,15 \\
\hline NGC 1353 & $\mathrm{Sbc}(\mathrm{r})$ & $\ldots$ & 0.11 & 11.22 & -20.32 & 19.31 & 7,15 \\
\hline NGC 1398 & SBab(r) & Bar & 0.05 & 9.64 & -21.47 & 16.26 & $7,8,15$ \\
\hline NGC 1425 & $\mathrm{Sb}(\mathrm{r})$ & $\ldots$ & 0.04 & 10.95 & -20.50 & 19.11 & 8,15 \\
\hline NGC 1512 & $\mathrm{SBb}(\mathrm{rs})$ & Bar & 0.04 & 10.44 & -19.56 & 9.85 & $8,14,15$ \\
\hline NGC 1566 & $\operatorname{Sbc}(s)$ & $\ldots$ & 0.03 & 9.69 & -20.75 & 12.08 & $2,7,14$ \\
\hline NGC 2775 & $\mathrm{Sa}(\mathrm{r})$ & $\ldots$ & 0.14 & 10.22 & -21.35 & 19.23 & $7,15,18$ \\
\hline NGC 2787 & $\mathrm{SB} 0 / \mathrm{a}$ & Bar & 0.43 & 10.72 & -19.86 & 10.69 & 7,15 \\
\hline NGC 2835 & $\mathrm{SBc}(\mathrm{rs})$ & Bar & 0.33 & 10.42 & -19.25 & 7.35 & 4,7 \\
\hline NGC 2841 & $\mathrm{Sb}$ & $\ldots$ & 0.05 & 9.31 & -21.12 & 11.91 & $1,6,10,12,14,18,19$ \\
\hline NGC 2880 & SB0 & Bar & 0.11 & 11.71 & -20.10 & 21.88 & $8,15,18$ \\
\hline NGC 2903 & $\mathrm{Sc}(\mathrm{s})$ & Bar & 0.10 & 8.98 & -20.14 & 6.35 & $8,10,15,18,19$ \\
\hline NGC 2950 & RSB0 & Bar & 0.06 & 11.04 & -20.80 & 22.80 & $7,10,15,18$ \\
\hline NGC 3031 & $\mathrm{Sb}(\mathrm{r})$ & $\ldots$ & 0.27 & 6.77 & -21.45 & 3.91 & $5,6,14,16,18$ \\
\hline NGC 3115 & So & $\ldots$ & 0.16 & 8.58 & -21.51 & 9.68 & $1,6,14,15$ \\
\hline NGC 3166 & $\mathrm{Sa}(\mathrm{s})$ & Oval & 0.10 & 10.23 & -20.93 & 16.22 & $7,15,18$ \\
\hline NGC 3169 & $\mathrm{Sb}(\mathrm{r})$ & $\ldots$ & 0.10 & 10.46 & -20.69 & 16.22 & $7,8,15,18$ \\
\hline NGC 3185 & $\mathrm{SBa}(\mathrm{s})$ & Bar & 0.09 & 12.08 & -19.36 & 18.56 & $8,10,15,18$ \\
\hline NGC 3198 & $\mathrm{Sc}(\mathrm{s})$ & $\ldots$ & 0.04 & 10.37 & -19.50 & 9.24 & $8,12,18,19$ \\
\hline NGC 3245 & S0 & $\ldots$ & 0.08 & 11.24 & -20.34 & 19.89 & $8,15,18$ \\
\hline NGC 3259 & $\operatorname{Sbc}(r)$ & $\ldots$ & 0.05 & 12.66 & -19.59 & 27.54 & $8,10,15,18$ \\
\hline NGC 3277 & $\mathrm{Sa}(\mathrm{r})$ & $\ldots$ & 0.09 & 11.68 & -19.90 & 19.89 & $7,10,15,18$ \\
\hline NGC 3338 & $\operatorname{Sbc}(s)$ & $\ldots$ & 0.10 & 10.82 & -20.73 & 19.42 & $8,15,18$ \\
\hline NGC 3351 & $\mathrm{SBb}(\mathrm{r})$ & Bar & 0.09 & 9.86 & -19.48 & 7.06 & $3,7,10,15,18,19$ \\
\hline NGC 3368 & $\mathrm{Sab}(\mathrm{s})$ & Oval & 0.08 & 9.13 & -21.61 & 13.53 & $7,8,10,15,18$ \\
\hline NGC 3423 & $\mathrm{Sc}(\mathrm{s})$ & $\ldots$ & 0.10 & 10.68 & -19.80 & 11.95 & 7,18 \\
\hline NGC 3521 & $\mathrm{Sbc}(\mathrm{s})$ & $\ldots$ & 0.19 & 9.31 & -21.19 & 11.48 & $3,7,15$ \\
\hline NGC 3593 & Sa pec & $\ldots$ & 0.07 & 10.67 & -18.57 & 6.83 & $7,15,18$ \\
\hline NGC 3627 & $\mathrm{Sb}(\mathrm{s})$ & Bar & 0.11 & 8.95 & -20.33 & 6.83 & $7,8,12,15,18$ \\
\hline NGC 3642 & $\mathrm{Sb}(\mathrm{r})$ & $\ldots$ & 0.04 & 10.79 & -21.45 & 27.54 & 7,18 \\
\hline NGC 3885 & $\mathrm{Sa}$ & $\ldots$ & 0.24 & 10.97 & -21.18 & 24.08 & 7,15 \\
\hline NGC 3898 & $\mathrm{Sa}$ & $\ldots$ & 0.07 & 10.71 & -20.85 & 19.88 & $7,10,15,18$ \\
\hline NGC 3992 & $\mathrm{SBb}(\mathrm{rs})$ & Bar & 0.10 & 9.79 & -21.04 & 14.03 & $7,15,18$ \\
\hline NGC 4030 & $\operatorname{Sbc}(r)^{*}$ & $\ldots$ & 0.09 & 10.22 & -21.57 & 21.89 & $7,15,18$ \\
\hline NGC 4062 & $\mathrm{Sc}(\mathrm{s})$ & $\ldots$ & 0.08 & 11.09 & -18.96 & 9.87 & $7,15,18$ \\
\hline NGC 4152 & $\mathrm{Sc}(\mathrm{r})$ & $\ldots$ & 0.11 & 12.05 & -20.53 & 31.15 & 7,10 \\
\hline NGC 4203 & S0 & $\ldots$ & 0.04 & 10.91 & -20.03 & 15.14 & 7,18 \\
\hline NGC 4245 & $\mathrm{SBa}(\mathrm{s})$ & Bar & 0.07 & 11.25 & -18.80 & 9.87 & $7,15,18$ \\
\hline NGC 4258 & $\mathrm{Sb}(\mathrm{s})$ & Oval & 0.05 & 8.39 & -20.97 & 7.28 & $5,7,15$ \\
\hline NGC 4260 & $\mathrm{SBa}(\mathrm{s})$ & Bar & 0.08 & 11.84 & -20.71 & 31.25 & 7,18 \\
\hline NGC 4274 & $\mathrm{Sa}(\mathrm{sr})$ & Oval & 0.17 & 10.31 & -19.84 & 9.87 & $5,15,18$ \\
\hline NGC 4314 & $\mathrm{SBa}(\mathrm{rs})$ pec & Bar & 0.08 & 10.63 & -19.42 & 9.87 & $7,10,15,18$ \\
\hline NGC 4340 & RSB0 & Bar & 0.09 & 11.20 & -20.47 & 20.79 & $5,15,18$ \\
\hline NGC 4371 & SB0(r) & Bar & 0.12 & 10.84 & -20.12 & 14.68 & $7,15,18$ \\
\hline NGC 4379 & S0 & $\ldots$ & 0.08 & 11.78 & -19.06 & 14.19 & 7,18 \\
\hline NGC 4380 & $\mathrm{Sab}(\mathrm{s})$ & $\ldots$ & 0.08 & 11.70 & -19.97 & 20.79 & $7,15,18$ \\
\hline NGC 4394 & $\mathrm{SBb}(\mathrm{sr})$ & Bar & 0.10 & 10.88 & -20.07 & 14.80 & $8,10,15,18$ \\
\hline NGC 4448 & $\mathrm{Sa}$ & Bar & 0.08 & 10.95 & -19.10 & 9.87 & $7,15,18$ \\
\hline NGC 4489 & S0** & $\ldots$ & 0.09 & 12.36 & -19.00 & 17.86 & $\mathrm{KFCB}^{\mathrm{e}}$ \\
\hline NGC 4501 & $\operatorname{Sbc}(s)$ & $\ldots$ & 0.13 & 9.66 & -22.55 & 26.05 & $7,10,15,19$ \\
\hline NGC 4536 & $\operatorname{Sbc}(s)$ & Oval & 0.06 & 10.84 & -21.24 & 25.30 & $7,12,15,18$ \\
\hline NGC 4564 & $\mathrm{~S} 0 * *$ & $\ldots$ & 0.12 & 11.25 & -19.74 & 15.00 & $\mathrm{KFCB}^{\mathrm{e}}$ \\
\hline NGC 4569 & $\mathrm{Sab}(\mathrm{s})$ & Bar & 0.15 & 9.50 & -22.24 & 20.79 & $7,10,15,18$ \\
\hline NGC 4570 & S0 & $\ldots$ & 0.07 & 10.66 & -20.56 & 16.98 & $\mathrm{KFCB}^{\mathrm{e}}$ \\
\hline NGC 4579 & $\mathrm{Sab}(\mathrm{s})$ & Bar & 0.14 & 9.90 & -21.83 & 20.79 & $7,12,15,18$ \\
\hline NGC 4639 & $\mathrm{SBb}(\mathrm{r})$ & Bar & 0.09 & 11.40 & -20.27 & 20.79 & $7,8,15,18$ \\
\hline NGC 4660 & S0** & $\ldots$ & 0.11 & 11.38 & -19.27 & 12.80 & $\mathrm{KFCB}^{\mathrm{e}}$ \\
\hline NGC 4698 & $\mathrm{Sa}$ & Oval & 0.09 & 10.58 & -22.30 & 36.09 & $7,10,15,18$ \\
\hline NGC 4725 & $\mathrm{SBb}(\mathrm{r})$ & Bar & 0.04 & 9.43 & -21.22 & 13.24 & $7,10,12,15$ \\
\hline NGC 4736 & RSab(s) & Oval & 0.06 & 8.28 & -18.27 & 1.99 & $7,10,12,18,19$ \\
\hline
\end{tabular}


Table 2

(Continued)

\begin{tabular}{lcccrcrc}
\hline \hline Identifier & $\begin{array}{c}\text { Hubble } \\
\text { type }\end{array}$ & $\begin{array}{c}\text { Driving } \\
\text { mechanism }\end{array}$ & $\begin{array}{c}a_{V}{ }^{\mathrm{b}} \\
(\mathrm{mag})\end{array}$ & $\begin{array}{c}V \\
(\mathrm{mag})\end{array}$ & $\begin{array}{c}M_{V} \\
(\mathrm{mag})\end{array}$ & $\begin{array}{c}\text { Distance }^{\mathrm{c}} \\
(\mathrm{Mpc})\end{array}$ & Data sources $^{\mathrm{d}}$ \\
\hline NGC 4772 & $\mathrm{Sa}(\mathrm{r}) *$ & $\mathrm{Bar}$ & 0.09 & 11.22 & -19.51 & 13.43 & 7,18 \\
NGC 4826 & $\mathrm{Sab}(\mathrm{s})$ & $\ldots$ & 0.14 & 8.57 & -20.94 & 7.48 & $6,7,10,13,15$ \\
NGC 4941 & $\mathrm{Sab}(\mathrm{s})$ & Oval & 0.12 & 11.29 & -17.41 & 5.22 & 7,15 \\
NGC 5055 & $\mathrm{Sbc}(\mathrm{s})$ & $\ldots$ & 0.06 & 8.67 & -21.46 & 10.36 & $7,11,15,18,19$ \\
NGC 5194 & $\mathrm{Sbc}(\mathrm{s})$ & $\ldots$ & 0.12 & 8.38 & -20.94 & 6.94 & $7,12,10,15$ \\
NGC 5273 & $\mathrm{S0/a}$ & $\ldots$ & 0.03 & 11.45 & -19.68 & 16.52 & $7,10,15,18$ \\
NGC 5879 & $\mathrm{Sb}(\mathrm{s})$ & $\ldots$ & 0.04 & 11.31 & -19.38 & 13.45 & $7,15,18$ \\
NGC 5970 & $\mathrm{SBbc(r)}$ & $\mathrm{Bar}$ & 0.14 & 10.88 & -21.34 & 26.00 & $8,9,18$ \\
NGC 6744 & $\mathrm{Sbc}(\mathrm{r})$ & $\mathrm{Bar}$ & 0.14 & 7.84 & -21.93 & 8.43 & 4,7 \\
NGC 6946 & $\mathrm{Sc}(\mathrm{s})$ & $\ldots$ & 1.13 & 8.57 & -21.33 & 5.67 & $7,10,12,15,17$ \\
NGC 7177 & $\mathrm{Sab}(\mathrm{r})$ & $\mathrm{Bar}$ & 0.24 & 11.30 & -20.10 & 17.08 & $7,8,9,15$ \\
NGC 7217 & $\mathrm{Sb}(\mathrm{r})$ & $\ldots$ & 0.29 & 10.07 & -21.23 & 15.92 & $8,15,20$ \\
NGC 7457 & $\mathrm{S} 0$ & $\ldots$ & 0.17 & 11.44 & -19.34 & 13.24 & $7,10,15$ \\
NGC 7743 & $\mathrm{SBa}$ & Bar & 0.23 & 11.34 & -20.47 & 20.70 & $7,10,15$ \\
\hline
\end{tabular}

Notes.

${ }^{\text {a }}$ Hubble types are taken from Sandage \& Bedke (1994).

${ }^{\mathrm{b}}$ Galactic extinction are taken from Schlegel et al. (1998).

${ }^{c}$ Distances are taken from Tully \& Fisher (1988).

${ }^{\mathrm{d}}$ Refers to number in the first column of Table 1.

${ }^{\mathrm{e}}$ Profiles are taken from Kormendy et al. (2008).
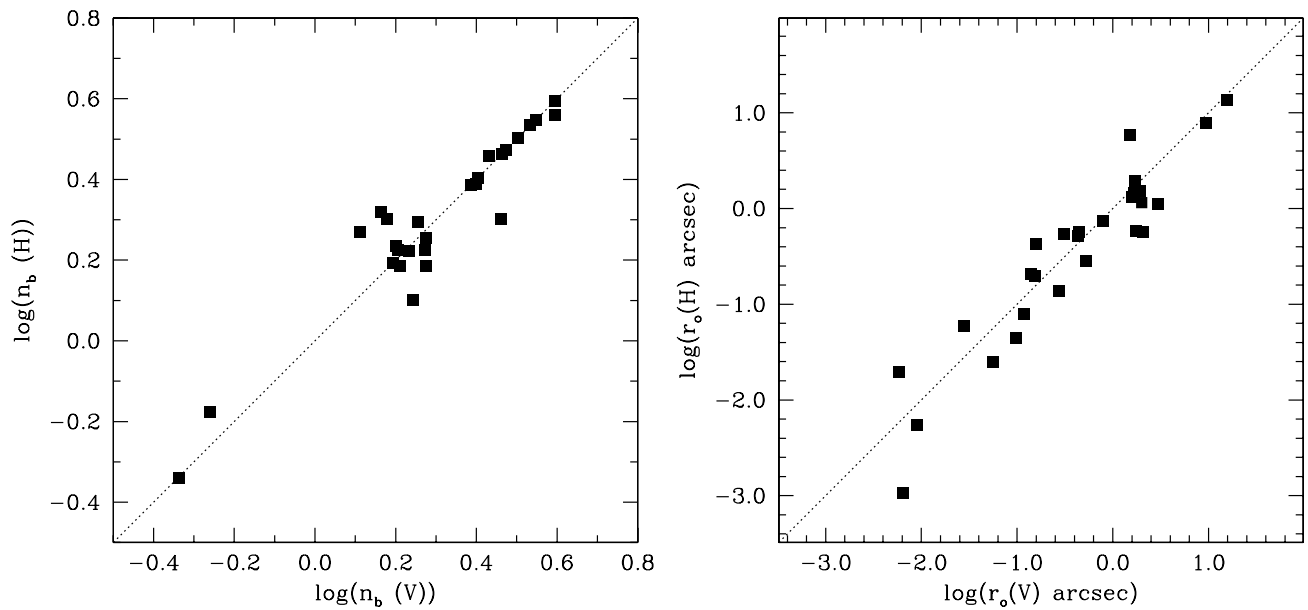

Figure 3. Comparison of structural parameters $\left(r_{0}\right.$ and $\left.n_{b}\right)$ derived from bulge-disk decompositions in the $H$-band to those in the $V$-band. The identity relation is marked by the dashed line.

to $V$-band filters. Note first that classical bulges (for example NGC 1398, NGC 2775, NGC 2880, and NGC 3115, all in the rightmost column of the first page of the figure) have a smooth stellar light profile. There is no reason evident in the images to think that any of these galaxies harbors a pseudobulge. The bulges fit the description of E-type galaxies. On the other hand, NGC 4030 shows a face-on nuclear spiral (bottom row on the second page). The spiral dominates the radial profile for more than a kiloparsec. NGC 4736 (fourth page, second row) also has a nuclear spiral pattern but also has a nuclear bar; note how the spiral arms seem pinched vertically in the image. Moellenhoff et al. (1995) study this nuclear bar in more detail using dynamical modeling. NGC 4371 (third page, middle row) is an S0 galaxy with at least one nuclear bar. NGC 3351 (second page, third row) has a prominent nuclear ring that heavily distorts the surface brightness profile (see Figure 4); this nuclear ring is quite well known (Sandage 1961). The bulge of NGC 2903 (first page, bottom row) shows a chaotic nuclear region; it appears nearly spiral but is not regular enough over a significant radial range to call it a nuclear spiral.
In this paper, we classify bulges with near- $V$-band images (F547M, F555W, and F606W). Thus our method is subject to the effects of dust obscuration. However, the structures used to identify pseudobulges are usually experiencing enhanced star-formation rates (Fisher 2006). Pseudobulges are, therefore, easier to detect in the optical band passes where the massto-light ratios are more affected by young stellar populations, rather than in the near infrared where the effects of dust are less pronounced. It is important to note that classical bulges may have dust in their center, as do many elliptical galaxies (Lauer et al. 2005). In fact, many classical bulges shown in Figure 2 have some dust lanes in their bulges. The presence of dust alone is not enough to classify a galaxy as containing a pseudobulge; instead, it must be of a disk-like nature.

\subsection{Data Sources and Surface Photometry}

As stated in the introduction, the Sérsic function provides better fits and more information over two-parameter fitting functions describing surface brightness profiles of bulges and elliptical galaxies. However, this information comes at the 
price of more detailed observations. Saglia et al. (1997) show that replacing an $r^{1 / 4}$ model with a Sérsic model makes little difference in residuals, unless one has data with high dynamic range in radius. Further, the coupling between parameters in the Sérsic function can be quite high (Graham \& Colless 1997). Thus again, it is necessary to fit the decomposition (Equation (1)) to large radial range in order to minimize these degeneracies. For each galaxy we therefore combine multiple data sources together: high-resolution HST imaging in the center, and groundbased wide-field images covering the outer disk. Comments on data sources follow. Table 1 lists the sources of data used for each galaxy.

All profiles contain HST data sources. PC 2 data have a small field of view $\left(\sim 18 \times 18 \mathrm{arcsec}^{2}\right)$; thus, it is critical to supplement PC 2 data with wide-field data. Advanced Camera for Surveys (ACS)/WFC has proven to be an excellent instrument for obtaining large radial fitting range. It provides a reasonable sized field of view $\left(\sim 100 \times 100 \operatorname{arcsec}^{2}\right)$ at high spatial resolution $\left(0.049\right.$ arcsec pixel $\left.^{-1}\right)$.

For as many galaxies as possible we obtain wide-field images from the Prime-Focus-Camera on the McDonald $0.8 \mathrm{~m}$ telescope. This instrument provides a large unvignetted field of view $\left(45 \times 45 \mathrm{arcmin}^{2}\right)$ and a single CCD detector. Therefore, we can more accurately carry out sky-subtraction. These data generally are the deepest. We also include images from the Sloan Digital Sky Survey (SDSS) (Adelman-McCarthy et al. 2006), 2MASS, and the Isaac Newton Group (ING) Archive. Individual data sources are noted in Table 1.

All raw data (McDonald $0.8 \mathrm{~m}$ and ING data) are biassubtracted, flat-fielded, and illumination corrected. We subtract the sky background by fitting a plane to a smoothed version of the image where the galaxy and bright stars have been removed.

We calculate Johnson $V$-band magnitude zero points using the transformations in Holtzman et al. (1995) for the WFPC 2 images and Sirianni et al. (2005) for the ACS images. SDSS $\mathrm{g}$ and $\mathrm{r}$ profiles are converted to a single $V$-band profile for each galaxy using the transformations in Smith et al. (2002). Other profiles are simply shifted to match in surface brightness. These transformations are derived on galactic disk stars, not galaxies. Further, the calculations require color information. We use colors from Hyper-LEDA, which refer to colors of the entire galaxies, and the galaxies in our sample most certainly have nonzero color gradients. Therefore, the absolute values of surface brightness in this paper are not expected to be consistent to more than $0.3 \mathrm{mag}$. However, this does not affect our conclusions which are based on the structure in the profiles and not on absolute magnitude. We check that our total magnitudes are consistent with those published in the RC 3 and Hyper-LEDA.

We use the isophote fitting routine of Bender \& Moellenhoff (1987). We identify and mask interfering foreground objects in each image. Then we fit ellipses to isophotes by least squares. Here, isophotes are sampled by 256 points equally spaced in an angle $\theta$ relating to polar angle by $\tan \theta=(a / b) \tan \phi$, where $\phi$ is the polar angle and $b / a$ is the axial ratio. The software determines six parameters for each ellipse: relative surface brightness, center position, major and minor axis lengths, and position angle along the major axis.

\subsection{Bulge-Disk Decomposition}

We carry out a bulge-disk decomposition on each galaxy in our sample by fitting Equation (1) to the major axis surface brightness profiles. Our decomposition code is also used by
Kormendy et al. (2008) to fit S0 and E-type galaxies with the disk component "turned off." The average root-meansquared (rms) deviation is $\sim 0.09 \pm 0.03 \mathrm{mag} \operatorname{arcsec}^{-2}$. The largest deviation of any of the data from its fitted profile is $0.18 \mathrm{mag} \operatorname{arcsec}^{-2}$.

The parameters determined in bulge-disk decomposition with a Sérsic model bulge are coupled. MacArthur et al. (2003) show that if the bulge is sufficiently large and the resolution is sufficiently small, initial parameter estimates do not affect the final fit too much. For the most part, this is true. Yet, our experience is that initial parameter estimates may still affect the resulting fit. For each galaxy, we begin with a large parameter range that is symmetric about the initial guess. Then we refit the galaxy iteratively adjusting the range of allowed parameters to be narrower. This results in slight changes of best-fit values with lower $\chi^{2}$ than without this iteration. The details of each profile are used to decide the width of available parameter space if each parameter is given. Typically, the available range for $n_{b}$ is $\Delta n_{b} \sim 2-3$.

The decomposition is carried out on a major axis profile using the mean isophote brightness. It does not take ellipticity into account during the fitting. However, these galaxies are known to have varying ellipticity profiles (Kormendy 1993; Fathi \& Peletier 2003). Thus, we take the mean ellipticity for each component and adjust the luminosity accordingly: $L=(1-\bar{\epsilon}) L_{\text {fit }}$. The radius of the component is defined as the radius range within which that component dominates the light of the profile. We also adjust all magnitude-dependent quantities (luminosity and surface brightness) for Galactic reddening according to Schlegel et al. (1998). We use the distances of Tully \& Fisher (1988). We do not make any corrections for extinction within the galaxies being studied.

We carry out the bulge-disk decompositions in the $V$-band. This ensures that our results will remain applicable to large surveys commonly done in the optical bands (e.g., SDSS). However, the radial variation in age and metallicity that exists within galaxies may bias the parameters of bulge-disk decomposition. Carollo et al. (2002) show that the centers of intermediatetype galaxies contain significant structure in $V-I$ color maps, and this variation occurs on scales smaller than the bulge. Thus, there is doubt as to whether the parameters derived on profiles in the middle optical band passes truly reflect the properties of the stellar mass distribution.

It is beyond the scope of this paper to investigate the correlations of colors with Sérsic parameters. Yet, as a check of the stability of structural quantities compared to color gradients, we carry out bulge-disk decompositions on all galaxies in our sample that have archival NICMOS images in the filter F160W ( $H$-band). We supplement those data with ground-based wide-field data. For this purpose we use mainly 2MASS data and any $H$-band data available in NASA/IPAC Extragalactic Database (NED). We then compare the fit parameters in $V$ to those in $H$. The results are shown in Figure 3. In those parameters which do not depend on magnitude $\left(r_{0}\right.$ and $\left.n_{b}\right)$ there is little difference. The average difference is $\Delta_{V-H} n_{b} \sim-0.03$ and $\Delta_{V-H} \log \left(r_{0}\right) \sim 0.07$. This is similar to the results of other papers that have done decompositions at multiple wavelengths (e.g., Graham 2001; MacArthur et al. 2003).

In Figure 4, we show four examples of our photometry and the bulge-disk decompositions. For each galaxy we show an ellipticity profile, a residual profile, and a surface brightness profile along with the decomposition determined by fitting Equation (1) to the galaxy's surface brightness profile. These 

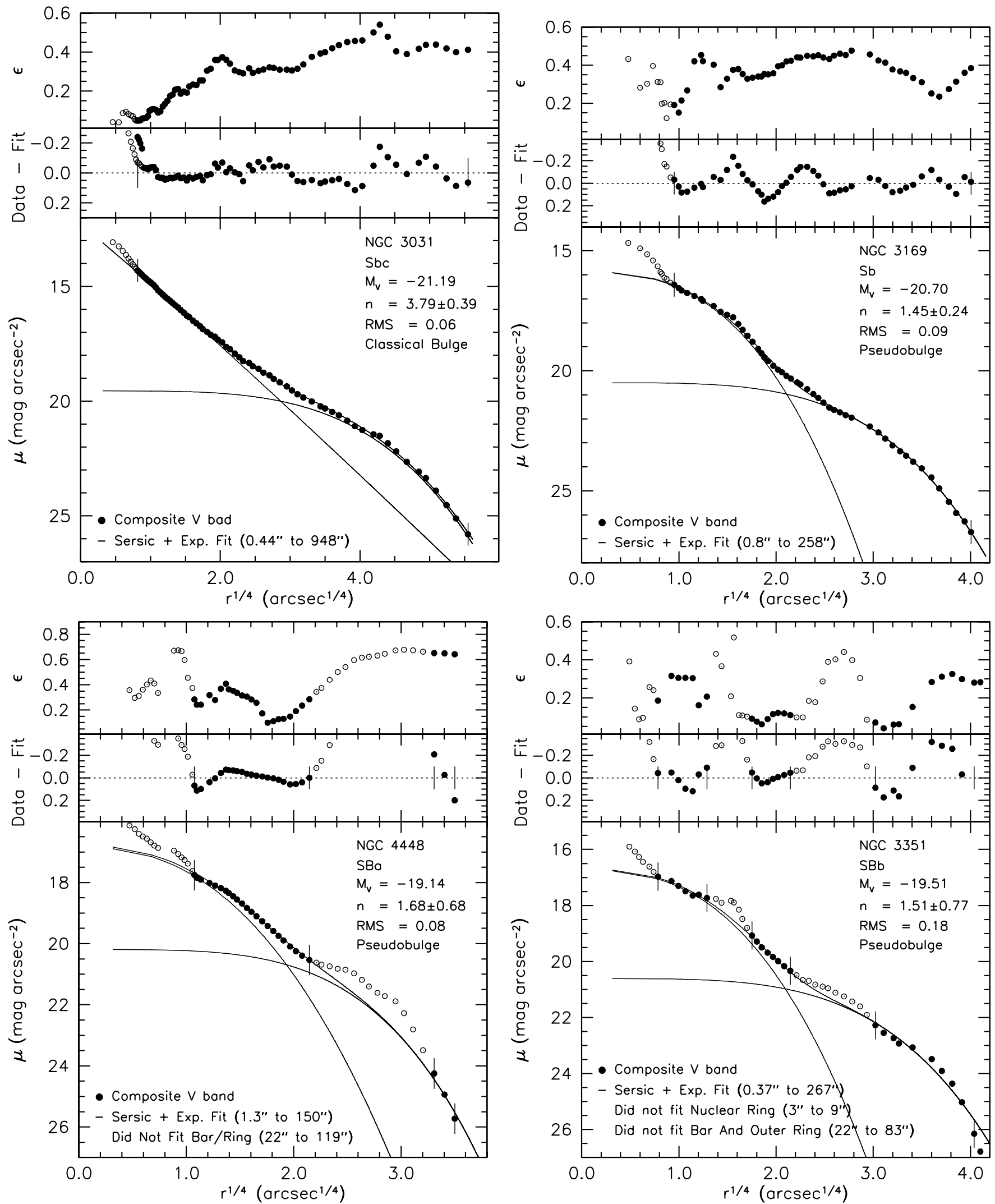

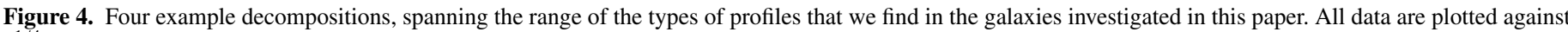

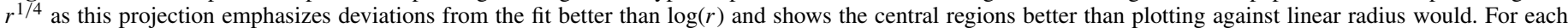

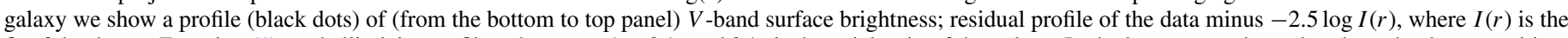

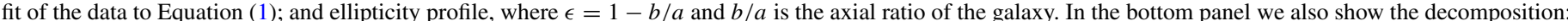

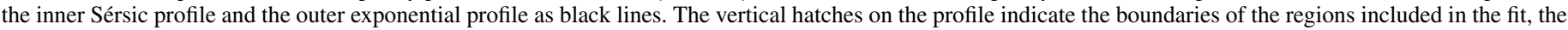

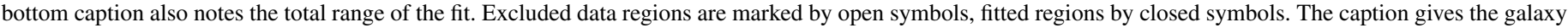
name, the Hubble type, the total absolute magnitude, the Sérsic index of the bulge, and the rms deviation of the fit to the fit region of the data. 
Table 3

Bulge and Disk Parameters

\begin{tabular}{|c|c|c|c|c|c|c|c|c|c|c|c|}
\hline lentifier & $\begin{array}{c}\text { Bulge } \\
\text { type }\end{array}$ & $\begin{array}{c}\text { Bulge } \\
\text { morphology }\end{array}$ & $\mathrm{rms}$ & $\begin{array}{c}\mu_{o}(V) \\
\left(m a g \operatorname{arcsec}^{-2}\right)\end{array}$ & $\begin{array}{c}r_{e} \\
(\operatorname{arcsec})\end{array}$ & $n_{b}$ & $\begin{array}{c}\mu_{D}(V) \\
\left(m a g \operatorname{arcsec}^{-2}\right)\end{array}$ & $\begin{array}{c}h \\
(\operatorname{arcsec})\end{array}$ & $\left\langle\epsilon_{b}\right\rangle$ & $\left\langle\epsilon_{D}\right\rangle$ & $r_{b=d}$ \\
\hline GC 613 & $\mathrm{p}$ & $\mathrm{nB}$ & 0.17 & $16.41 \pm 0.42$ & $5.49 \pm 2.97$ & $23 \pm 0.40$ & $19.47 \pm 0.25$ & $33.47 \pm 1.75$ & $0.39 \pm 0.20$ & $0.48 \pm 0.04$ & 8.73 \\
\hline GC 628 & $\mathrm{p}$ & $\mathrm{nS}$ & 0.04 & $17.65 \pm 0.20$ & $13.83 \pm 3$ & $5 \pm$ & $20.29 \pm 0.09$ & $33 \pm$ & $9 \pm 0.07$ & $3 \pm 0.01$ & 4.29 \\
\hline NGC 1022 & $\mathrm{p}$ & $\mathrm{nB}$ & 0.07 & $14.12 \pm 0.54$ & $1.06 \pm 0.57$ & $3 \pm 0.36$ & & $19.21 \pm 0.63$ & & $0.15 \pm 0.02$ & 10.03 \\
\hline NGC 1300 & $\mathrm{p}$ & $\mathrm{nS}$ & 0.07 & & $41 \pm 2.57$ & $1 \pm 0.39$ & & $58.27 \pm 3.03$ & & & 9.03 \\
\hline NGC 1313 & $\mathrm{p}$ & $\mathrm{nP}$ & .11 & $7.83 \pm 0.55$ & $.57 \pm 1.26$ & $78 \pm 0.62$ & $18.85 \pm 0.10$ & $51.98 \pm 3.90$ & $42 \pm 0.21$ & $0.46 \pm 0.05$ & 2.21 \\
\hline NGC 1325 & $\mathrm{n}$ & $\mathrm{nS}$ & 09 & $8.27 \pm 0.81$ & $4.72 \pm 4.12$ & $50 \pm 0.79$ & $20.24 \pm 0.11$ & $36.61 \pm 1.27$ & $36 \pm 0.19$ & $0.61 \pm 0.01$ & 3.02 \\
\hline NGC 1353 & $\mathrm{p}$ & $\mathrm{nS}$ & 0.13 & $15.02 \pm 0.47$ & $2.60 \pm 2.07$ & $79 \pm 0.41$ & $19.54 \pm 0.15$ & $26.10 \pm 1.18$ & $45 \pm 0.15$ & $0.46 \pm 0.01$ & 4.42 \\
\hline NGC 1398 & c & $\mathrm{C}$ & 0.13 & $13.04 \pm 0.69$ & $23.22 \pm 33.90$ & $3 \pm 0.68$ & $20.97 \pm 0.60$ & $66.72 \pm 10.87$ & $13 \pm 0.06$ & $0.22 \pm 0.01$ & 20 \\
\hline NGC 1425 & $\mathrm{c}$ & $\mathrm{C}$ & 0.12 & $13.27 \pm 1.36$ & $8.50 \pm 8.48$ & $5 \pm 1.12$ & $20.28 \pm 0.45$ & $41.16 \pm 5.89$ & $29 \pm 0.11$ & $0.50 \pm 0.01$ & 9.03 \\
\hline NGC 1512 & $\mathrm{p}$ & $\mathrm{nR}$ & 08 & $6.39 \pm 0.29$ & $7.85 \pm 3.23$ & $56 \pm 0.28$ & $20.24 \pm 0.16$ & $46.09 \pm 2.49$ & $0.10 \pm 0.08$ & $0.45 \pm 0.01$ & 8 \\
\hline NGC 1566 & $\mathrm{p}$ & $\mathrm{nS}$ & 0.07 & $16.61 \pm 1.64$ & $8.90 \pm 6.10$ & $58 \pm 1.11$ & $19.80 \pm 0.27$ & $51.45 \pm 4.29$ & $0.19 \pm 0.12$ & $0.30 \pm 0.02$ & 10.53 \\
\hline NGC 2775 & c & $\mathrm{C}$ & 0.07 & $13.62 \pm 0.51$ & $32.11 \pm 18.42$ & $80 \pm 0.39$ & $21.02 \pm 0.31$ & $45.30 \pm 5.41$ & $0.12 \pm 0.06$ & $0.18 \pm 0.00$ & 39.99 \\
\hline NGC 2787 & $\mathrm{p}$ & $\mathrm{nB}$ & 0.08 & $16.86 \pm 0.50$ & $9.12 \pm 5.55$ & $24 \pm 0.33$ & & $25.89 \pm 1.11$ & & $0.37 \pm 0.02$ & 14.44 \\
\hline SC 2835 & $\mathrm{p}$ & & & $19.91 \pm 0.75$ & $34 \pm 6$ & $9 \pm 1$ & & -1 & $0.27 \pm 0.17$ & $0.29 \pm 0.00$ & 5.31 \\
\hline NGC 2841 & $\mathrm{c}$ & $\mathrm{C}$ & & & & 7 & & & & 02 & 13 \\
\hline NGC 2880 & $\mathrm{c}$ & $c$ & 0.04 & $13.42 \pm 0.32$ & $13.03 \pm 12.89$ & $0 \pm 0.31$ & $21.08 \pm 0.30$ & $22.84 \pm 1$ & $20 \pm 0.09$ & $0.35 \pm 0.02$ & 10 \\
\hline 903 & $\mathrm{p}$ & $\mathrm{nP}$ & 12 & $17.58 \pm 0.16$ & $9 \pm 0$ & $2 \pm 0.13$ & $20.29 \pm 0.30$ & $47 \pm 6$ & $1 \pm 0.17$ & $0.50 \pm$ & 12.03 \\
\hline & $\mathrm{p}$ & $\mathrm{nB}$ & 08 & $4.04 \pm 0.62$ & $4.67 \pm 3.24$ & $0 \pm$ & $20.50 \pm 0.25$ & $26.04 \pm$ & $28 \pm 0.12$ & $0.35 \pm 0.01$ & 14.44 \\
\hline & c & $\mathrm{C}$ & 0.06 & $12.34 \pm 0.43$ & $75.05 \pm 68.09$ & $9 \pm 0.39$ & $19.55 \pm 0.13$ & $161.38 \pm 4.97$ & $22 \pm 0.07$ & $0.41 \pm 0.00$ & 68.08 \\
\hline 115 & $\mathrm{c}$ & $\mathrm{C}$ & 0.09 & $10.76 \pm 0.41$ & $30.98 \pm 2$ & $9 \pm 0$ & & $2 \pm$ & $56 \pm 0.11$ & $0.60 \pm 0.04$ & 54.52 \\
\hline NGC 3166 & $\mathrm{p}$ & $\mathrm{nS}$ & 0.07 & $0 \pm 0.58$ & $0 \pm 1$ & $6 \pm 1$ & $8 \pm 0$ & $6 \pm$ & $1 \pm 0.23$ & $0.27 \pm 0.03$ & 7.22 \\
\hline NGC 3169 & $\mathrm{p}$ & $\mathrm{nS}$ & 09 & $9 \pm 0.34$ & $5 \pm 3$ & $6 \pm 0.24$ & & $6 \pm$ & $34 \pm 0.12$ & $0.35 \pm 0.06$ & 19.85 \\
\hline GC 3185 & $\mathrm{p}$ & & 12 & \pm 0.60 & $8 \pm$ & $9 \pm$ & 19 & \pm & 12 & $0.39 \pm 0.01$ & 3.74 \\
\hline GC 3198 & $\mathrm{p}$ & $\mathrm{nS}$ & 03 & $19.18 \pm 0.94$ & $33.36 \pm$ & $9 \pm$ & $21.60 \pm 1.08$ & 135 & 14 & $0.61 \pm 0.03$ & 22.25 \\
\hline NGC 3245 & c & $\mathrm{C}$ & 07 & $4+043$ & 12 & +1 & 2 & & & $0.44 \pm$ & 8 \\
\hline 59 & $\mathrm{p}$ & $\mathrm{nS}$ & & 17 & 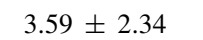 & 4 & & & 19 & 0.36 & 2.62 \\
\hline & $\mathrm{c}$ & $\mathrm{C}$ & & $12.12 \pm 0.88$ & 1 & 5 & & & & 0.25 & 38 \\
\hline NGC & $\mathrm{p}$ & & & 17. & \pm 2 & $1 \pm$ & 2 & $7 \pm$ & 6 & 01 & 3 \\
\hline & $\mathrm{p}$ & & 0.18 & 5 & \pm & $1 \pm$ & & $1 \pm$ & $8 \pm$ & $0.19 \pm$ & 13.84 \\
\hline & $\mathrm{p}$ & & & 30 & \pm & $1 \pm$ & & \pm & $8 \pm$ & $0.32 \pm$ & 33.58 \\
\hline & $\mathrm{p}$ & $\mathrm{nS}$ & 0.07 & 19 & \pm 4 & $7 \pm$ & & 06 & $15 \pm$ & $0.17 \pm$ & 4.37 \\
\hline & c & $\mathrm{C}$ & 13 & 9 & - & \pm & & & & 0.46 & .83 \\
\hline C 3593 & $\mathrm{p}$ & $\mathrm{C}$ & 14 & \pm 0.69 & \pm & \pm & 52 & 1 & 17 & $0.60=$ & 17.29 \\
\hline NGC 3627 & $\mathrm{p}$ & & 1 & $5 \pm 0.85$ & $57+2$ & $0 \pm$ & 0.28 & O & $8 \pm 0.11$ & $0.54 \pm 0.01$ & 14.39 \\
\hline NGC 3642 & $\mathrm{p}$ & & & $5+087$ & 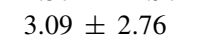 & 1 & 18 & 2 & .08 & $0.07=$ & 6.92 \\
\hline NGC 3885 & $\mathrm{p}$ & IIS & 16 & $14.47 \pm 0.95$ & $5.37 \pm 6.46$ & $8 \pm 0.82$ & $4 \pm 0$ & $2 \pm 2$ & $34 \pm 0.16$ & $0.55 \pm 0.01$ & 11.83 \\
\hline NGC 3898 & c & $\mathrm{C}$ & & $12.21 \pm 1.28$ & $18.50 \pm 15.98$ & $4 \pm 0.88$ & & $9 \pm 1$ & $32 \pm 0.10$ & $0.43 \pm 0.00$ & 40.59 \\
\hline & $\mathrm{c}$ & $\mathrm{C}$ & & $14.29 \pm 1.04$ & $18.82 \pm 13$ & $5 \pm 0$ & & $9 \pm 1$ & $22 \pm 0.09$ & $0.46 \pm$ & 12.33 \\
\hline NGC 4030 & $\mathrm{p}$ & & & $16.51 \pm 0.98$ & $11.28 \pm 7.07$ & & & -10.0 & $3 \pm 0.09$ & 01 & 10.03 \\
\hline & $\mathrm{p}$ & & & & & 4 & & & 0 & 2 & 2.21 \\
\hline & $P$ & & 7 & & \pm & \pm & 1 & \pm & $8 \pm$ & 02 & 2.81 \\
\hline 203 & $\mathrm{c}$ & $\mathrm{C}$ & 11 & 22 & \pm & $4 \pm$ & 20 & \pm & $1 \pm$ & $0.11 \pm$ & 14.89 \\
\hline 245 & $\mathrm{p}$ & $\mathrm{nR}$ & 08 & \pm 0.74 & + & $0+$ & \pm 0.52 & 56 & $16 \pm 0.09$ & $0.29 \pm 0.02$ & 14.04 \\
\hline NGC 4258 & $\mathrm{p}$ & $\mathrm{nS}$ & .04 & $14.35 \pm 0.61$ & $14.98 \pm 8.02$ & $0 \pm$ & $3 \pm 0.14$ & $3 \pm 6.26$ & 0.15 & $0.51=$ & 25.26 \\
\hline NGC 4260 & c & $\mathrm{C}$ & 07 & $.09 \pm 0.63$ & $15.31 \pm 30.17$ & $1 \pm 0.66$ & $20.11 \pm 0.31$ & $8 \pm 1.74$ & $23 \pm 0.09$ & $0.47 \pm 0.00$ & 7.97 \\
\hline NGC 4274 & $\mathrm{p}$ & & 14 & $15.89 \pm 0.50$ & $7.45 \pm 6.82$ & $2 \pm 0.48$ & $20.04 \pm 0.19$ & $18 \pm 2.81$ & $43 \pm 0.16$ & $0.40 \pm 0.05$ & 10.83 \\
\hline NGC 4314 & $\mathrm{p}$ & & 16 & $14.64 \pm 0.81$ & $5 \pm 6.48$ & $37 \pm 0.78$ & $19.09 \pm 0.20$ & $5 \pm 0.79$ & $18 \pm 0.11$ & $0.25 \pm 0.02$ & 6.62 \\
\hline NGC 4340 & $\mathrm{p}$ & & 07 & $15.25 \pm 0.88$ & $6.46 \pm 3.06$ & $00 \pm 0.67$ & $20.63 \pm 0.36$ & $30.97 \pm 3.60$ & $.10 \pm 0.06$ & $0.31 \pm 0.01$ & 11.73 \\
\hline NGC 4371 & $\mathrm{p}$ & & 07 & $15.42 \pm 0.24$ & $7.74 \pm 3$ & $02 \pm 0$ & $21.00 \pm 0.13$ & $44.95 \pm 1.63$ & $30 \pm 0.14$ & $0.35 \pm 0.04$ & 17.44 \\
\hline NGC 4379 & $\mathrm{c}$ & $\mathrm{C}$ & 4 & $13.78 \pm 0.35$ & $15.35 \pm 1$ & $8 \pm 0$ & 20. & \pm 1 & \pm 0.09 & 01 & \\
\hline NGC 4380 & $\mathrm{p}$ & & & & & & & & 6 & & 4 \\
\hline NGC & 1 & & & & & 土 & & & 8 & & 9.63 \\
\hline NGC 4448 & $P$ & & 88 & \pm 0.80 & $10.69 \pm$ & $8 \pm$ & $20.19 \pm 0.72$ & $2 \pm 4.74$ & $26 \pm 0.12$ & .03 & 14.04 \\
\hline NGC 4489 & $\mathrm{c}$ & $\mathrm{C}$ & 05 & $12.95 \pm 0.40$ & $600-$ & $8 \pm$ & $20.81 \pm 0.25$ & $7 \pm 1.07$ & $09 \pm 0.07$ & $0.10 \pm$ & 9.78 \\
\hline NGC 4501 & $\mathrm{p}$ & $\mathrm{nS}$ & 16 & $17.74 \pm 0.92$ & $6.96 \pm 5.42$ & $0.91 \pm 0.63$ & $19.34 \pm 0.17$ & $46.81 \pm 2.88$ & $.17 \pm 0.16$ & $0.48 \pm 0.00$ & 4 \\
\hline NGC 4536 & $\mathrm{p}$ & $\mathrm{nS}$ & 0.12 & $15.47 \pm 0.40$ & $4.33 \pm 2.90$ & $1.88 \pm 0.35$ & $20.99 \pm 0.16$ & $70.61 \pm 7.24$ & $.41 \pm 0.14$ & $0.53 \pm 0.01$ & 9.63 \\
\hline NGC 4564 & c & $\mathrm{C}$ & 04 & $12.04 \pm 0.78$ & $8.67 \pm 5.06$ & $3.70 \pm 0.66$ & $19.21 \pm 0.25$ & $18.73 \pm 1.47$ & $24 \pm 0.08$ & $0.54 \pm 0.02$ & 13.09 \\
\hline NGC 4569 & $\mathrm{p}$ & $\mathrm{nB}$ & 09 & $12.13 \pm 1.12$ & $1.64 \pm 1.49$ & $1.90 \pm 0.56$ & $19.77 \pm 0.11$ & $69.15 \pm 3.36$ & $.48 \pm 0.16$ & $0.56 \pm 0.01$ & 6.67 \\
\hline NGC 4570 & $\mathrm{c}$ & $\mathrm{C}$ & 3 & $11.85 \pm 0.57$ & $10.14 \pm 11.87$ & $65 \pm 0$ & $18.67 \pm 0.18$ & $22.90 \pm 0.98$ & $31 \pm 0.10$ & $0.65 \pm 0.01$ & 8.83 \\
\hline NGC 4579 & $\mathrm{p}$ & $\mathrm{C}$ & 0.08 & $15.41 \pm 1.31$ & $17.48 \pm 15.02$ & $2.04 \pm$ & $20.19 \pm 0$ & $47.62 \pm 2.82$ & $0.22 \pm 0.12$ & $0.23 \pm 0.01$ & 26.06 \\
\hline NGC 4639 & $\mathrm{p}$ & $\mathrm{n}_{\mathrm{s}}$ & 0.1 & $16.06 \pm 0.89$ & $4.43 \pm 3.29$ & $1.64 \pm 0.90$ & $20.57 \pm 0.49$ & $26.97 \pm 4.22$ & $0.20 \pm 0.10$ & $0.37 \pm 0.01$ & 8.79 \\
\hline & $\mathrm{c}$ & $\mathrm{C}$ & & & & & & & & & \\
\hline NGC 4698 & $\mathrm{c}$ & C & & & $16.96 \pm$ & & & & & & 13.09 \\
\hline NGC 4725 & $\mathrm{c}$ & $\mathrm{C}$ & 0.07 & $13.77 \pm 1.01$ & $62.72 \pm 119.71$ & $3.19 \pm 0$ & $20.63 \pm 0.27$ & $8 \% .05 \pm$ & $0.14 \pm 0.07$ & .01 & 10.5 \\
\hline NGC 4736 & $\mathrm{p}$ & $\mathrm{nB}$ & 0.1 & $14.13 \pm 0.54$ & $9.68 \pm 7.23$ & $1.62 \pm 0.51$ & $18.08 \pm 0.76$ & $29.93 \pm 9.41$ & $0.17 \pm 0.09$ & $0.18 \pm 0.04$ & 17.89 \\
\hline
\end{tabular}


Table 3

(Continued)

\begin{tabular}{|c|c|c|c|c|c|c|c|c|c|c|c|}
\hline Identifier & $\begin{array}{c}\text { Bulge } \\
\text { type }\end{array}$ & $\begin{array}{c}\text { Bulge } \\
\text { morphology }\end{array}$ & $\mathrm{rms}$ & $\begin{array}{c}\mu_{o}(V) \\
\left(\mathrm{mag} \operatorname{arcsec}^{-2}\right)\end{array}$ & $\begin{array}{c}r_{e} \\
(\operatorname{arcsec})\end{array}$ & $n_{b}$ & $\begin{array}{c}\mu_{D}(V) \\
\left(\mathrm{mag} \operatorname{arcsec}^{-2}\right) \\
\end{array}$ & $\begin{array}{c}h \\
(\operatorname{arcsec})\end{array}$ & $\left\langle\epsilon_{b}\right\rangle$ & $\left\langle\epsilon_{D}\right\rangle$ & $r_{b=c}$ \\
\hline NGC 4772 & $\mathrm{c}$ & $\mathrm{C}$ & 0.07 & $14.50 \pm 0.72$ & $15.12 \pm 22.40$ & $3.20 \pm 0.57$ & $21.15 \pm 0.36$ & $44.70 \pm 5.00$ & $0.07 \pm 0.05$ & $0.38 \pm 0.03$ & 15 \\
\hline NGC 4826 & $\mathrm{p}$ & $\mathrm{nS}$ & 0.08 & $11.72 \pm 0.79$ & $16.71 \pm 21.46$ & $3.94 \pm 0.68$ & $18.89 \pm 0.14$ & $60.48 \pm 2.29$ & $0.24 \pm 0.08$ & $0.44 \pm 0.00$ & 11. \\
\hline NGC 5055 & $\mathrm{p}$ & $\mathrm{nS}$ & 0.08 & $17.33 \pm 0.55$ & $46.91 \pm 34.28$ & $1.84 \pm 0.49$ & $20.26 \pm \varepsilon$ & $99.75 \pm 173.35$ & $0.32 \pm 0.14$ & $0.44 \pm 0.01$ & 40. \\
\hline NGC 5194 & $\mathrm{p}$ & $\mathrm{nS}$ & 0.08 & $18.10 \pm 0.21$ & $13.83 \pm 1.95$ & $0.55 \pm 0.14$ & $19.87 \pm 0$ & $86.82 \pm 3.42$ & $0.16 \pm 0.12$ & 0.22 & 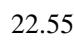 \\
\hline NGC 5273 & $\mathrm{c}$ & $\mathrm{C}$ & 0.09 & 12.94 & $5.32 \pm 13$ & $1 \pm$ & 1 & $21.16 \pm$ & 08 & 0.14 & $0 .{ }^{2}$ \\
\hline NGC 6744 & $\mathrm{c}$ & $\mathrm{C}$ & 0.06 & $14.81 \pm 0.62$ & $26.55 \pm 21.72$ & $2.53 \pm 0.59$ & $19.68 \pm 0.22$ & $134.85 \pm 15.12$ & $0.19 \pm 0.10$ & $0.45 \pm 0.03$ & 15 \\
\hline NGC 6946 & $\mathrm{p}$ & $\mathrm{nP}$ & 0.1 & $15.90 \pm 0.99$ & $4.41 \pm 4.47$ & $1.87 \pm 0.71$ & $20.57 \pm 0.13$ & $147.12 \pm 16.83$ & $0.37 \pm 0.14$ & $0.23 \pm 0.02$ & 7.2 \\
\hline NGC 7177 & $\mathrm{p}$ & $\mathrm{nS}$ & 0.08 & $16.60 \pm 0.28$ & $7.90 \pm 3.07$ & $1.51 \pm 0.36$ & $19.56 \pm 0.42$ & $18.84 \pm 1.56$ & $0.39 \pm 0.13$ & $0.31 \pm 0.00$ & 10.6 \\
\hline NGC 7217 & $\mathrm{p}$ & $\mathrm{nS}$ & 0.08 & $16.24 \pm 1.44$ & $27.38 \pm 33.54$ & $1.90 \pm 0.96$ & $20.47 \pm 0.53$ & $38.81 \pm 4.57$ & $0.08 \pm 0.07$ & $0.09 \pm 0.00$ & 19. \\
\hline NGC 7457 & $\mathrm{c}$ & $\mathrm{C}$ & 0.07 & $15.28 \pm 0.83$ & $6.28 \pm 4.88$ & $2.44 \pm 0.71$ & $20.02 \pm 0.19$ & $26.89 \pm 1.23$ & $0.25 \pm 0.12$ & $0.44 \pm 0.01$ & \\
\hline NGC 7743 & $\mathrm{c}$ & $\mathrm{C}$ & 0.08 & $11.28 \pm 0.87$ & $22.16 \pm 36.40$ & $5.12 \pm 0.49$ & $20.63 \pm 0.22$ & $24.89 \pm 1.98$ & $0.09 \pm 0.08$ & $0.24 \pm 0.04$ & 5 \\
\hline
\end{tabular}

examples are selected to show the range in typical profiles in this paper. The entire sample of fits is shown in the appendix.

The top two panels of Figure 4 show two galaxies (NGC 3031, left panel; NGC 3169, right panel) that are well described by an inner Sérsic function and an outer exponential disk. In both profiles the fit covers a dynamic range of $\sim 12 \mathrm{mag} \operatorname{arcsec}^{-2}$. NGC 3031 is well fitted (rms $=0.06 \mathrm{mag}$ $\left.\operatorname{arcsec}^{-2}\right)$ by Equation (1) over $\Delta \log (r / \operatorname{arcsec})=3.3$ and NGC 3169 for $\Delta \log (r / \operatorname{arcsec})=2.51$. Deviations are small, and there is little-to-no substructure evident in these profiles. Note that each bulge has a nuclear excess of light over the inward extrapolation of the fit and that this nucleus is excluded from the fit (vertical hatches indicate the beginning and end of the fit range and excluded data points are marked by open symbols).

Despite its successes, the Sérsic bulge plus outer exponential disk model of bulge-disk galaxies does not account for many features of galaxy surface brightness profiles. Disks of intermediate-type galaxies commonly have features such as bars, rings, and lenses (see Kormendy 1982 for a description of these). Further, Carollo et al. (2002) show that many bulges of early- and intermediate-type galaxies contain nuclei. The bottom panels of Figure 4 show two such examples of galaxy profiles with significant deviations from the fitted decompositions. NGC 4448 (bottom left) is an example of a barred galaxy in which the bar is an especially prominent perturbation to the outer exponential surface brightness profile. NGC 3351 (bottom right) is an example of a complicated surface brightness profile, with multiple substructures that are not well described by the smooth nature of the bulge-disk model used here. This galaxy contains a nuclear ring near $\sim 4$ arcsec, and a bar from about 20 to 80 arcsec. These galaxies are not well described by Equation (1), yet we do our best to decompose as many galaxies as possible.

Bars, rings, lenses, and similar features do not conform to the smooth nature of Equation (1), hence we carefully exclude regions of the profile perturbed by such structures from the fit. This is a risky procedure, as it requires selectively removing data from a galaxy's profile, and undoubtedly has an effect on the resulting parameters. We are often helped to identify bars by the structure of the ellipticity profile. Note the peak in ellipticity near 50 arcsec in NGC 3351. We try to err on the side of removing the fewest points possible. For those galaxies in which a bar is present, it is our assumption that removing the bar from the fit provides the best estimation of the properties of the underlying bulge and disk. We test our method by removing a typical number of points from a few galaxies with smooth profiles (NGC 2841, NGC 1425, NGC 4203, and NGC 1325). The result is that Sérsic index can vary as much as $\Delta n_{b} \sim 0.5$, and characteristic radius by $\Delta \log \left(r_{0}\right) \sim 0.5$. The variance of these two parameters is tightly coupled and this is reflected by the uncertainty. Central surface brightness was mostly unaffected. If a region is not included in a fit we show that in the figure by using open symbols. A detailed discussion of the effects of bars and other features on the surface brightness profiles is given in the appendix.

\section{STRUCTURAL PROPERTIES OF PSEUDOBULGES AND CLASSICAL BULGES}

\subsection{Bulge Prominence of Classical and Pseudobulges Along the Hubble Sequence}

The primary distinction between most previous studies and this one is that we do not treat bulges as a homogeneous set of objects. Here, we report on how morphologically-identified pseudobulges and classical bulges, taken to be distinct entities, behave in the parameters obtained from bulge-disk decomposition. Further we wish to know if the Sérsic index is able to distinguish these two separate bulge types as has been suggested by many authors and not yet systematically tested.

Figure 5 shows how the bulge-to-total ratio (luminosity of the bulge, divided by total luminosity of the fit; hereafter $B / T$ ) and the ratio of the bulge half-light radius $\left(r_{e}\right)$ to the scale length of the outer disk $(h)$ both correlate with Hubble type. We calculate the effective radius as

$$
r_{e}=\left(b_{n}\right)^{n} r_{0},
$$

where $b_{n}$ is a proportionality constant whose expansion is given in MacArthur et al. (2003). In all figures, the red circles represent classical bulges and the blue crosses represent pseudobulges. In Figure 5, the connected black squares show the average for each Hubble type. There are only two S0/a $(T=0)$ galaxies; thus the dip in the average of $B / T$ could merely be small number statistics. Also, a few of the Sc $(T=5)$ galaxies are not shown due to their very small $B / T$.

As shown in the top panel of Figure 5, the bulge-to-total ratio is a decreasing function of Hubble type. This is not surprising as 


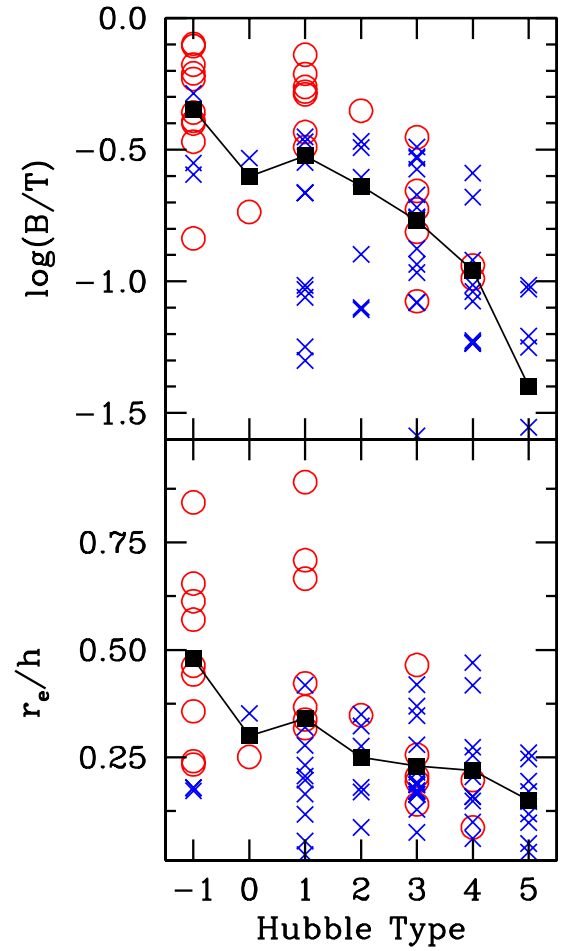

Figure 5. Bulge-to-total ratio, $B / T$, top, and the ratio of the effective radius to the disk scale length, $r_{e} / h$, bottom, of classical bulges (red circles) and pseudobulges (blue $\times$ 's) plotted against Hubble types. The black squares, connected by the black line, represent the average for all bulges (both pseudoand classical) in each type.

$B / T$ is part of the original classification criteria. This behavior has been found by many authors, for a detailed study see Graham (2001). This at least confirms that our decomposition method is sound and able to recover the well-established correlation.

Pseudobulges and classical bulges overlap in the range of bulge prominence, as shown in the left panel of Figure 6 . The average classical bulge has $\langle B / T\rangle=0.41$. The width of the distribution is 0.22 . The average pseudobulge has $\langle B / T\rangle=$ 0.16 . The width of the distribution is 0.11 . Pseudobulges are on average smaller fractions of the total luminosity of the galaxy in which they reside, yet there is significant overlap (see also Drory \& Fisher 2007). This is no surprise, we would expect pseudobulges to be smaller as they are thought to be products of secular evolution of the disk; hence their luminosity is expected to be limited to some fraction of the luminosity of the disk. On the other hand, classical bulges form independently of their disk, presumably through major mergers. The full range values of $B / T$ are therefore possible.

NGC 2950 is a pseudobulge with $B / T=0.51$. It is hard to believe that secular evolution can drive half of the stellar mass into the central region of a galaxy. This galaxy is an SB0 with a prominent nuclear bar and nuclear ring, also noticed by Erwin \& Sparke (2003). Thus, by our definition it is a good pseudobulge candidate. The next most prominent pseudobulge has $B / T=0.35$; NGC 2950 is more than one standard deviation away in the distribution of pseudobulge $B / T$ from the next most prominent pseudobulge. We feel that NGC 2950 is an exceptional case and should not be taken as a normal pseudobulge. That it is an S0 galaxy strengthens this interpretation. The unusually large $B / T$ may be a result of the processes which made the galaxy $\mathrm{S} 0$ (for example gas stripping by ram pressure and/or harassment; see Moore et al. 1996), rather than secular evolution. Also, our analysis may be an oversimplification of the population of bulges, in that composite systems ("bulges" with both a pseudobulge and a classical bulge) may exists. This could artificially increase the $B / T$ ratio.

Courteau et al. (1996) show that the ratio of scale lengths for galaxies when fitted with a double exponential (bulge and disk) is tightly coupled. MacArthur et al. (2003) find, with fits to Sérsic plus outer exponential profiles in galaxies spanning Hubble type $\mathrm{Sab}$ to $\mathrm{Sd}$, that the coupling is $\left\langle r_{e} / h\right\rangle=0.22 \pm 0.09$ and that the ratio becomes smaller toward later Hubble types (see also the lower panel of Figure 5). It appears that the correlation of $r_{e} / h$ with Hubble type may be driven primarily by the number of classical bulges in each Hubble type; this statement is very uncertain, however, due to small numbers in each Hubble type.

The right panel of Figure 6 shows the distribution of $r_{e} / h$ for the galaxies in our sample (the red lines are for classical bulges and the blue lines represent the distribution of pseudobulges). We find that $\left\langle r_{e} / h\right\rangle=0.28 \pm 0.28$ for all galaxies in our sample and that $r_{e} / h$ decreases toward later Hubble types. The average is higher and the scatter is larger than in MacArthur et al. (2003), most likely because our sample targets earliertype galaxies. Considering bulge types separately, we find that for pseudobulges $\left\langle r_{e} / h\right\rangle=0.21 \pm 0.10$, and for classical

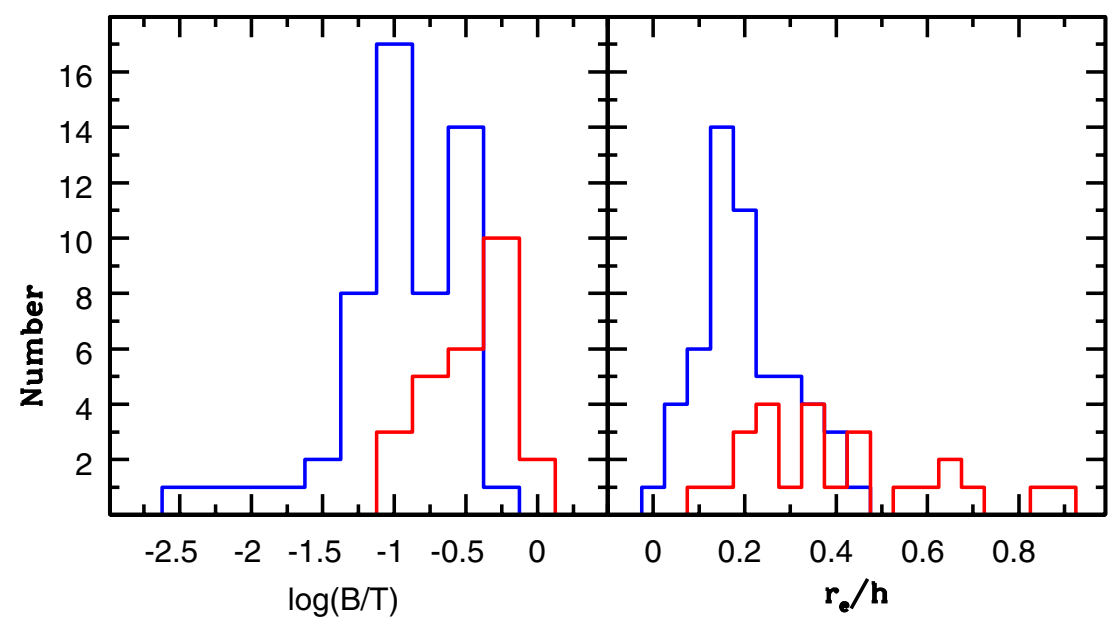

Figure 6. Histogram of $\log (B / T)$ (left) and $r_{e} / h$ (right). Bin spacing is $\delta \log (B / T)=0.25$ and $\delta\left(r_{e} / h\right)=0.05$. The blue lines represent pseudobulges and the red lines are for classical bulges. 


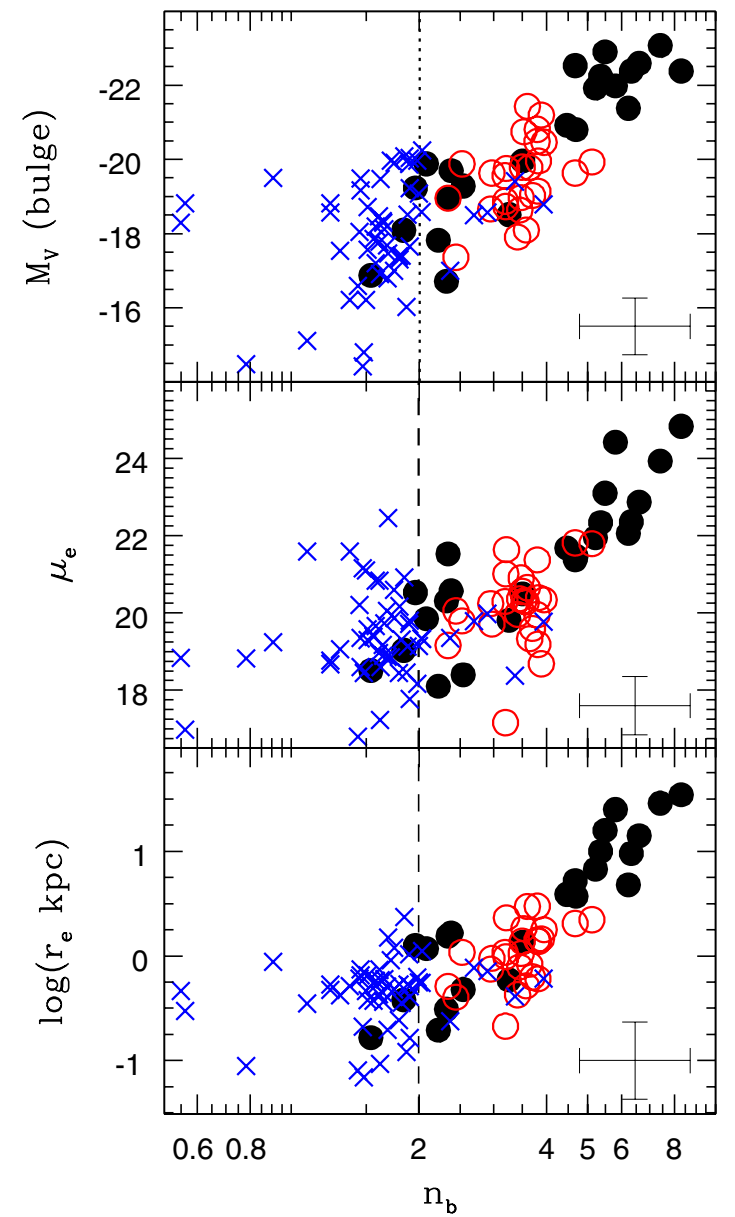

Figure 7. Correlation of the absolute magnitude $\left(M_{V}\right)$, half-light radius $\left(r_{e}\right)$, and surface brightness at the half-light radius $\left(\mu_{e}\right)$ of bulges with bulge Sérsic index. Pseudobulges are represented as blue crosses, and classical bulges as red circles. We also show elliptical galaxies from Kormendy et al. (2008) for comparison, represented by black filled circles. The average uncertainty of the parameters of all bulges is represented by the error bars in the bottom right corner of each panel.

bulges $\left\langle r_{e} / h\right\rangle=0.45 \pm 0.28$. The distribution of $r_{e} / h$ for pseudobulges is clearly much narrower than that of the classical bulges. Furthermore, $r_{e}$ and $h$ do not appear to be correlated in classical bulges.

Our finding that the half-light radius of pseudobulges is well correlated with the scale length of the outer disk, while the scale length of classical bulges is not correlated with that of the disk, is consistent with the interpretation that $r_{e} \propto h$ is due to a secular formation of pseudobulges. However, inspection of Figure 6 shows that the range of $r_{e} / h$ over which we find classical bulges is large and overlaps with the pseudobulges significantly. Thus, $r_{e} / h$ may not be a good diagnostic tool for identifying pseudobulges and classical bulges; however, the finding that the scale length of the disk is correlated with the size of its pseudobulge but not correlated with classical bulges is a strong indication that there indeed is a physical difference between the formation mechanisms of different bulge types.

\subsection{The Sérsic Index in Bulges}

Figure 7 shows the correlation of bulge properties with the shape of the surface brightness profile measured by the Sérsic index, $n_{b}$, of those bulges. We find that no classical bulge has Sérsic index less than 2 , and very few pseudobulges $(\sim 10 \%)$

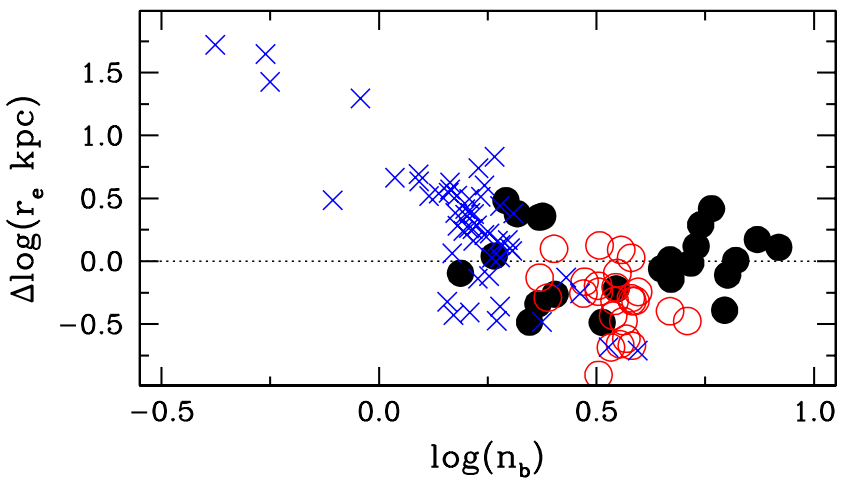

Figure 8. Residuals of pseudobulges (crosses), classical bulges (open circles), and elliptical galaxies (closed circles) from a fit to the elliptical galaxies given by $\log \left(r_{e} / \mathrm{kpc}\right)=2.9 \log \left(n_{b}\right)-1.2$.

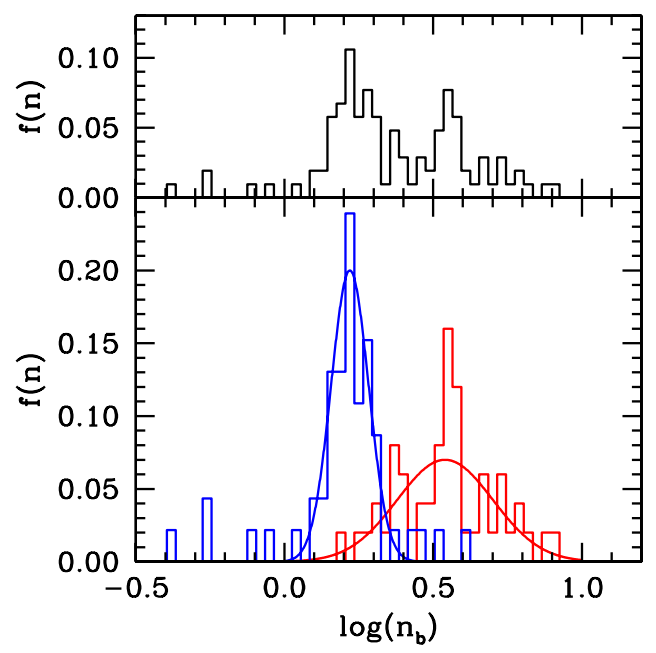

Figure 9. Histograms of bulge Sérsic index, $\log \left(n_{b}\right)$, by bulge type. The top panel shows the histogram of all objects. The bottom panel shows pseudobulges (blue lines with mode $\log \left(n_{b}\right) \sim 0.22$ ) and classical bulges + elliptical galaxies (red lines, mode $\log \left(n_{b}\right) \sim 0.54$ ). We also overplot Gaussian distributions that fit the histograms. Histograms are normalized by the total number of galaxies in each group, so that each bin reflects the frequency of Sérsic index within that object class.

have Sérsic index greater than 2. The correlations of Sérsic index with other structural properties $\left(r_{e}, \mu_{e}\right.$, and $\left.M_{v}\right)$ appear to be different for pseudobulges and classical bulges. This suggests that the Sérsic index may be used to distinguish pseudobulges from classical bulges.

The top panel of Figure 7 shows the correlation of Sérsic index with absolute $V$-band bulge magnitude for pseudobulges, classical bulges, and elliptical galaxies. Classical bulges fit well within the correlation set by elliptical galaxies. They cover a range of Sérsic index from $n_{b} \sim 2-4.5$, and their absolute magnitude covers the range $M_{V} \sim-17.5$ to -21 . Note that the Sérsic index of classical bulges is normally smaller than $n=4$, suggesting their similarity to the low-luminosity ellipticals discussed by Kormendy et al. (2008). Classical bulges, yet again, resemble little elliptical galaxies that happen to be surrounded by a disk. The mean pseudobulge absolute magnitude is $\sim 1.2 \mathrm{mag}$ fainter than the average classical bulge magnitude, and the faintest bulges are all pseudobulges. Yet, pseudobulges do not have to be faint: classical bulges are not much brighter than the brightest pseudobulges. Our data cannot show if the brightest pseudobulges are bright because of the nature of their stellar population, or because of greater stellar 
mass. Nonetheless, pseudobulges are not merely a change in observed properties at low magnitude. Finally, we note that the overall shape of the $M_{V}-n$ correlation for the super-set of systems represented in Figure 7 is similar to those found in previous studies (Graham 2001; Balcells et al. 2003; de Jong et al. 2004).

The bottom two panels of Figure 7 show the correlation of Sérsic index with the half-light quantities (radius containing half the light, $r_{e}$, and surface brightness at that radius, $\mu_{e}$ ) of pseudobulges, classical, bulges, and elliptical galaxies. The results are similar to those in the $M_{V}-n$ plane. Classical bulges exist within the bounds set by elliptical galaxies, more specifically by low-luminosity ellipticals. Pseudobulges, on the other hand, populate the small $n$ extreme of this correlation. The smallest objects, in radius, are pseudobulges. Yet, pseudobulges in general are not that much smaller on average than classical bulges and low-luminosity ellipticals: the mean effective radius of pseudobulges lies within the range of effective radii spanned by the classical bulges and elliptical galaxies.

To more strongly emphasize the difference between pseudobulges from classical bulges and ellipticals (hot stellar systems), we show the residuals of all galaxies to the Caon et al. (1994) relation $r_{e} \propto \log \left(n_{b}\right)$ in Figure 8 . We fit this correlation to the elliptical galaxies only, and the resulting relation is $\log \left(r_{e} / \mathrm{kpc}\right)=2.9 \log \left(n_{b}\right)-1.2$. E-type galaxies and classical bulges have similar amounts of scatter. Pseudobulges show a marked difference and systematically deviate further from the correlation as $n_{b}$ gets smaller whereas the scatter among classical bulges is contained within the region occupied by ellipticals. Thus, it appears that pseudobulges do not relate $n_{b}$ to $r_{e}$ in the same way as elliptical galaxies and classical bulges do. It is not clear from the bottom panel of Figure 7 whether Sérsic index correlates at all with effective radius for pseudobulges.

Inspection of Figure 7 shows that pseudobulges and classical bulges have separate distributions of bulge Sérsic index; the value that distinguishes the two types of bulges appears to be $n_{b} \sim 2$. Pseudobulges have Sérsic index smaller than $n_{b} \sim 2$; classical bulges have larger values than $n_{b} \sim 2$. We show this explicitly in Figure 9 which shows the distributions of $\log \left(n_{b}\right)$ for pseudobulges (blue lines) and the super-set of classical bulges and elliptical galaxies (red lines), both binned to $\delta \log \left(n_{b}\right)=0.03$. We also show a histogram of all objects (pseudobulges, classical bulges, and elliptical galaxies) counted together. Each histogram is normalized by the number of objects in that group, so that the counts reflect the frequency of Sérsic index within each class. Before discussing these distributions any further, we must strongly qualify this result. The sample studied in this paper is in no way complete. Thus, the relative abundance of pseudobulges and classical bulges cannot be determined from this distribution. However, we wish to study the distribution of Sérsic indices in different bulge types. We do the best we can with the data we have at present; when relevant, we attempt to point out how our sample selection may bias results. We note that distributions of bulge Sérsic indices in volume limited samples have been shown to be bimodal (Allen et al. 2006). We present any numbers derived from analysis of these distributions as preliminary.

The top panel of Figure 9 shows that the distribution of Sérsic indices are clearly bimodal. This bimodality is completely coincident with the dichotomy in bulge morphology. The average values of bulges Sérsic index for pseudobulges and classical bulges are $1.69 \pm 0.59$ and $3.49 \pm 0.60$, respectively, and the average of the super-set of classical bulges and elliptical

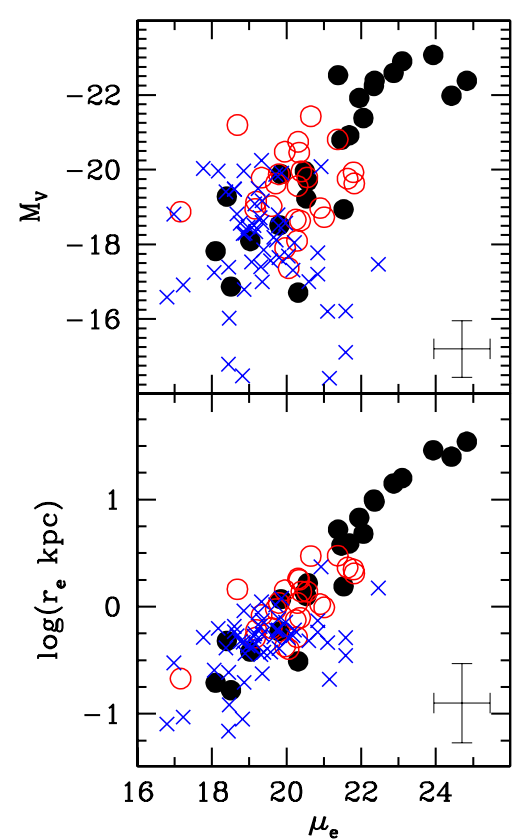

Figure 10. Two photometric projections of the fundamental plane. All symbols are the same as in Figure 7.

galaxies is $3.78 \pm 1.5$. The range of Hubble types chosen for this sample (S0-Sc) certainly biases the distribution of Sérsic index to higher values since the Sérsic index has been found to get smaller at later Hubble types (Graham 2001; MacArthur et al. 2003). It is worth noting that the average uncertainty of a bulge (pseudo- or classical) is comparable to the standard deviation of either of these distributions, $\left\langle\Delta n_{b}\right\rangle=0.60$. Inspection of Figure 7 shows that neither $\log \left(r_{e}\right)$ nor $\mu_{e}$ shows a bimodal distribution.

We fit a Gaussian to each histogram in Figure 9 and solve for the Sérsic index where the frequencies are equal. Because our sample is not volume limited, we weight the distributions such that the number of galaxies earlier than Hubble type Sc with pseudobulges is equal to the number of galaxies with classical bulges. We also solve for the case with $1 / 3$ pseudobulges, and finally $2 / 3$ pseudobulges. We find the critical Sérsic index to be $n_{\text {crit }}=2.2 \pm 0.1$.

Of the 52 pseudobulges, five have Sérsic index significantly above 2 (two pseudobulges have $n_{b} \sim 2$ ). The interested reader can inspect their surface brightness profile in the appendix, which shows fits to all surface brightness profiles. We take a few lines to discuss these galaxies here. NGC 4314 is an SBa galaxy with $n_{b}=2.37 \pm 0.78$; this fit appears good, although a significant amount of the profile is not included due to the outer bar and nuclear ring. NGC 4258 is an oval galaxy, with $n_{b}=2.69 \pm 0.48$. The outer oval ring affects the surface brightness profile of the outer disk, and thus the fit covers a narrow range. However, this is unlikely to affect the fit of the bulge too much, especially given the relatively low uncertainty in Sérsic index in this fit. NGC 3627 is an Sb galaxy with $n_{b}=$ $2.90 \pm 0.83$. Its bar is not easily detectable, and thus not removed from the fit, as we try not to remove any unnecessary points. NGC 3642 is an Sb galaxy with $n_{b}=3.37 \pm 0.61$, the profile does not go very deep, and may be allowing $n_{b}$ to be artificially high. Finally, NGC 4826 has the largest Sérsic index of any bulge classified as a pseudobulge, and the fit appears quite good, unlike the others. We take these galaxies as exceptions rather than the rule, future studies comparing alternative methods of 


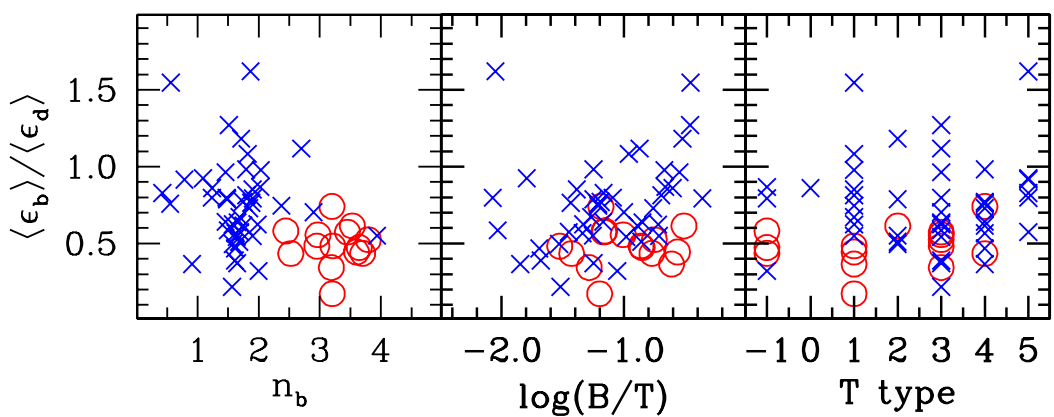

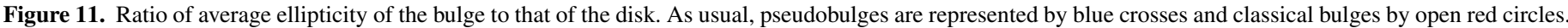
This ratio is plotted against Sérsic index of the bulge (left), bulge-to-total luminosity ratio (middle), and Hubble type (right).

pseudobulge detection (e.g., kinematics) will help shed light on their true nature. We remind the reader again that we are not classifying systems as composites, and these may have an effect. If for example a pseudobulge is embedded in a classical bulge (or vice versa), we might visually identify the pseudobulge even though the stellar light distribution is set by the classical bulge. Still, the dividing line of $n_{b} \sim 2$ is $90 \%$ successful at identifying pseudobulges, and thus is a good detector of pseudobulges.

\subsection{Fundamental Plane Projections}

Figure 10 shows the correlations of $\mu_{e}-r_{e}$ and $M_{v}-\mu_{e}$, two projections of the fundamental plane (Djorgovski \& Davis 1987; Dressler et al. 1987; Faber et al. 1989). The top correlation shows the magnitude versus surface brightness plane, and the bottom panel shows the $\mu_{e}-r_{e}$ relation (Kormendy 1977). While the $M_{V}$ versus $\mu_{e}$ plot has a lot of scatter, especially in the pseudobulges, the radius-surface brightness plane shows significantly less scatter. Yet in both of these fundamental plane projections, those bulges which are further from the correlations established by the elliptical galaxies are pseudobulges. For those pseudobulges that deviate from the fundamental plane correlation in Figure 10 that deviation is toward lower densities.

This behavior has been noticed in the past. Carollo (1999) found that exponential bulges are systematically lower in effective surface brightness than those better fit by an $r^{1 / 4}$ profile. We note that this could be an effect of only fitting either $r^{1 / 4}$ or exponentials rather than a Sérsic function to the bulge profiles. If a bulge is not completely exponential then it may force other parameters to compensate for the more restrictive parameterization of the profile shape. Falcón-Barroso et al. (2002) found that bulges which deviate from the edge-on projection of the fundamental plane are found in late-type (Sbc) galaxies. However, at least with the galaxies in our sample, we cannot say unambiguously that this is a function of differing bulge formation, because those bulges in Figure 10 that deviate significantly from the correlations defined by the elliptical galaxies have also very low $B / T$. Thus, it could be that the potential of the bulge is more affected by the outer disk.

In fact, when looking at the pseudobulges in Figure 10 alone, one would not infer the presence of a strong correlation of either magnitude or effective radius with surface brightness. This is another indication that pseudobulges and classical bulges are different classes of objects.

\section{FLATTENING OF CLASSICAL BULGES AND PSEUDOBULGES}

There has been very little work on the distribution of flattenings of bulges albeit the structures present in pseudobulges (e.g., nuclear spirals or nuclear bars) suggest that pseudobulges should have higher angular momentum and thus be flat stellar systems. However, the end products of secular evolution need not be flat (Kormendy 1993). Bar buckling and unstable disks can both drive stars higher above the plane of the disks (Pfenniger \& Norman 1990; Friedli 1999), thus creating a pseudobulge that is less flat than its associated outer disk. Nonetheless, the data of Kent (1985) show that many bulges have median flattenings that are similar or greater than the median of the outer disk, and that flat bulges are more common in late-type galaxies. Fathi $\&$ Peletier (2003) find a similar result that $\epsilon_{\text {bulge }} / \epsilon_{\text {disk }}>0.9$ in $36 \%$ of $\mathrm{S} 0-\mathrm{Sb}$ galaxies and $51 \% \mathrm{Sbc}-\mathrm{Sm}$ galaxies (where $\epsilon=1-b / a$ ). However, note that Möllenhoff \& Heidt (2001) do not find this result, they find very few bulges are as flat as disks in ground-based $J H K$ imaging.

Kormendy \& Kennicutt (2004) include the flattening of bulges (as manifest through the ratio of bulge ellipticity to that of the disk) in their list of preliminary criteria for identifying pseudobulges. We can test this hypothesis with our sample. Figure 11 shows the ratio of mean ellipticity of the bulge to that of the outer disk $\left(\left\langle\epsilon_{b}\right\rangle /\left\langle\epsilon_{d}\right\rangle\right)$ for galaxies in this study. We do not include galaxies with average disk ellipticity less than 0.2 , as face-on projections of galaxies do not allow the flattening to be determined. Also, galaxies with $B / T>0.5$ are removed; if the bulge dominates the entire potential it may set the shape of the disk and therefore affect the disk's ellipticity making it more like its own. To calculate the average ellipticity of the profile we only use the data points that are also included in the fit (filled circles in Figure 4 and in the appendix). It is a matter of interpretation as to what features are a part of pseudobulges, especially in light of the fact that some nuclei may be formed secularly along with the pseudobulge. However, as a matter of consistency we choose to focus only on those isophotes we call the "bulge" from the bulge-disk decompositions. The boundary between the bulge and disk is chosen as the radius at which the surface brightness of the Sérsic function equals the surface brightness of the exponential disk in the decomposition. Yet, in many galaxies contamination from the disk artificially raises the average ellipticity of the bulge. This contamination is evident in the ellipticity profile. Thus, we choose to average the bulge over a region in which there is little contamination present. Those radii are given in Table 2 .

The leftmost panel in Figure 11 compares $\left\langle\epsilon_{b}\right\rangle /\left\langle\epsilon_{d}\right\rangle$ to bulge Sérsic index for pseudobulges and classical bulges. It is quite evident that classical bulges, in our sample, are not as flat as pseudobulges. The average ratio of ellipticities of pseudobulges is $0.79 \pm 0.1$, whereas the average ratio for classical bulges is $0.49 \pm 0.14$. In fact, the flattest classical bulge $\left(\left\langle\epsilon_{b}\right\rangle /\left\langle\epsilon_{d}\right\rangle=\right.$ 0.75 ) is less flat than the average pseudobulge. 


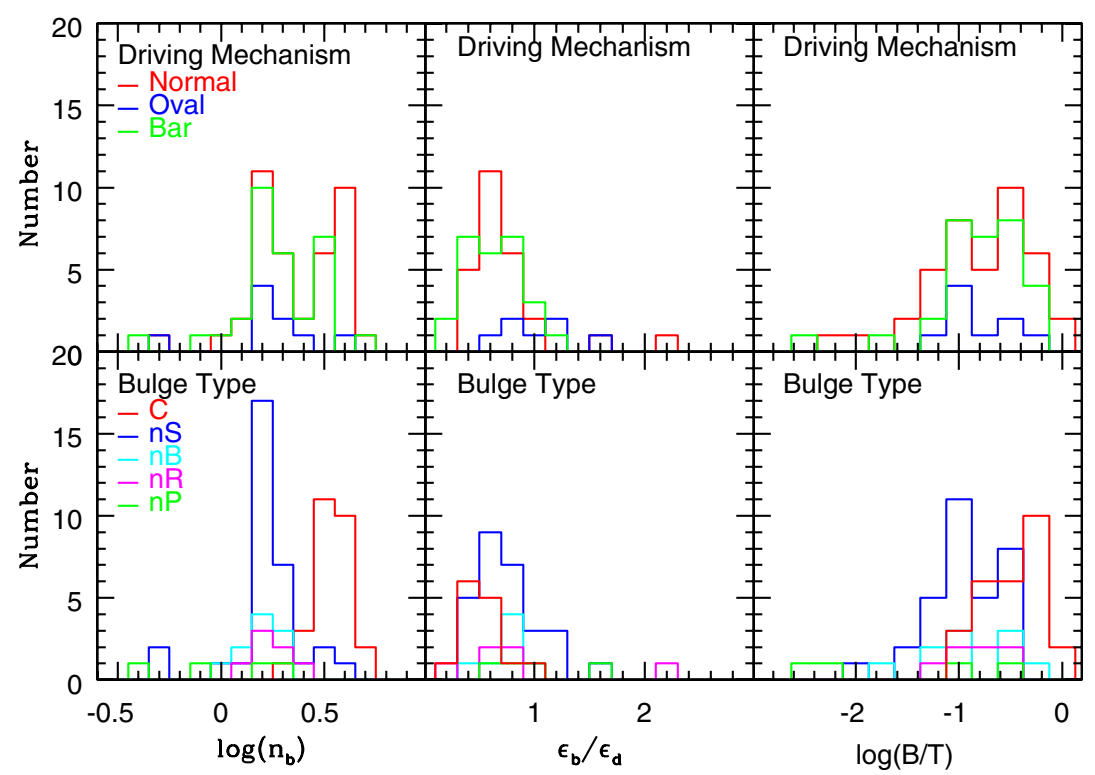

Figure 12. Bottom panels: the distribution of Sérsic index (bottom left), ratio of ellipticities (bottom middle), and $B / T$ (bottom right) for different bulge morphologies. The bulge types are denoted by nS: nuclear spiral, nB: nuclear bar, nR: nuclear Ring, nP: nuclear patchiness, and C: classical bulge. We also show the comparison of the presence of driving mechanism (N: no driver, O: oval, and B: bar) to the Sérsic index (top left), ratio of ellipticities (top middle), and $B / T$ (top right).

The middle and right panels of Figure 11 compare this ratio to the bulge-to-total ratio and Hubble type, respectively. Classical bulges show no obvious trend of $\left\langle\epsilon_{b}\right\rangle /\left\langle\epsilon_{d}\right\rangle$ with $B / T$ or Hubble type. They are tightly clustered about the mean value $\sim 0.6 \pm 0.1$. Pseudobulges however show a slight correlation of $\left\langle\epsilon_{b}\right\rangle /\left\langle\epsilon_{d}\right\rangle$ with $B / T$. This correlation indicates that more prominent (in $B / T$ ) pseudobulges are slightly flatter. However, the trend is weak and has large scatter. Finally, we find a similar result to Kormendy (1993) and Fathi \& Peletier (2003), namely that flatter bulges are more frequent in later-type galaxies. Furthermore, this trend only exists for pseudobulges.

Invariably the flattest bulges are pseudobulges. However, we do not wish to overstate this result. It is possible that asymmetric dust extinction leads to higher apparent flattening in the $V$-band. Future work at near-to-mid-IR wavelengths is likely to provide less ambiguous results.

\section{PSEUDOBULGES AS A CLASS OF OBJECTS}

In this paper we identify pseudobulges as having any, but not necessarily all, of several structures (nuclear bars, rings, and ovals). We treat bulges with these structures as a group. The motivation for doing so is that all these phenomena are similar to properties that are commonly associated with high-specific angular momentum systems. At least in the sense that they are the complement of classical bulges they can be treated as a group. However, here we have to ask the following question: do thus identified pseudobulges act as a single class of objects or do significant differences exist among the objects we are identifying as pseudobulges?

Figure 12 suggests an answer to this question. The left panels in the figure show the distribution of bulge properties for bulges that have smooth isophotes (C: classical), bulges with nuclear spirals (nS), bulges with nuclear bars (nB), bulges with nuclear rings (nR), and bulges with a chaotic nuclear patchiness that resembles late-type galaxies (nP). See Figure 2 for example images of each of these features. In Sérsic index, ellipticity ratio (bulge to disk), and bulge-to-total ratio there is no significant difference among the types of morphologies we call a pseudobulge.
Bulges with chaotic nuclear-disk-like patchiness (nP) seem to have smaller Sérsic index and are more flat. It is unclear why this might be. This may be driven by them having smaller $B / T$ and thus them being more affected by the disk potential. We also note that pseudobulges with $n_{b}>2$ are almost all in bulges with nuclear spirals. It is possible that these bulges are not truly a spiral, instead some other phenomenon, like contamination of the light by the outer disk, is causing us to identify them as pseudobulges. However, in each parameter the distinction appears to be between classical bulges and the rest of the objects, rather than among the objects we call pseudobulges.

We also show the Sérsic index, ellipticity ratio (bulge to disk), and bulge-to-total ratio as a function of secular driving mechanism. We separate galaxies as having no driving mechanism, an oval disk, or a bar. Note once again that we do not set grand design spiral as a class of object. There seems to be little differences in the averages, except ovaled galaxies have smaller Sérsic index and flatter bulges. The phenomenon of flat bulges does not appear to require the presence of a bar. Thus it is not likely that all flat bulges are small bulges stretched by a bar potential making them flat. Also note that the distributions of parameters for barred, oval, and normal spirals are roughly the same. Thus, it does not seem likely that our method of removing the bar is artificially changing the bulge-disk decomposition parameters.

\section{ENVIRONMENTAL EFFECTS}

Secular evolution is not the only theory for building bulges that look like disks. Other possibilities such as extremely gas-rich accretion events, distant gravitational encounters, or gravitational interactions with a cluster potential could all drive gas to the center of galaxies to increase $B / T$, as found by Mastropietro et al. (2005). Kannappan et al. (2004) find that blue bulges are statistically more likely to have companion galaxies within $100 \mathrm{kpc}$. Further, counter-rotating gas is frequently observed in spiral galaxies and is taken as a sign of the galaxy having accreted galaxies in their past. Yet, pseudobulges are much more common in late-type galaxies, and the wellknown morphology-density relation (Dressler 1980) shows that late-type galaxies are not found in dense environments. Is it 


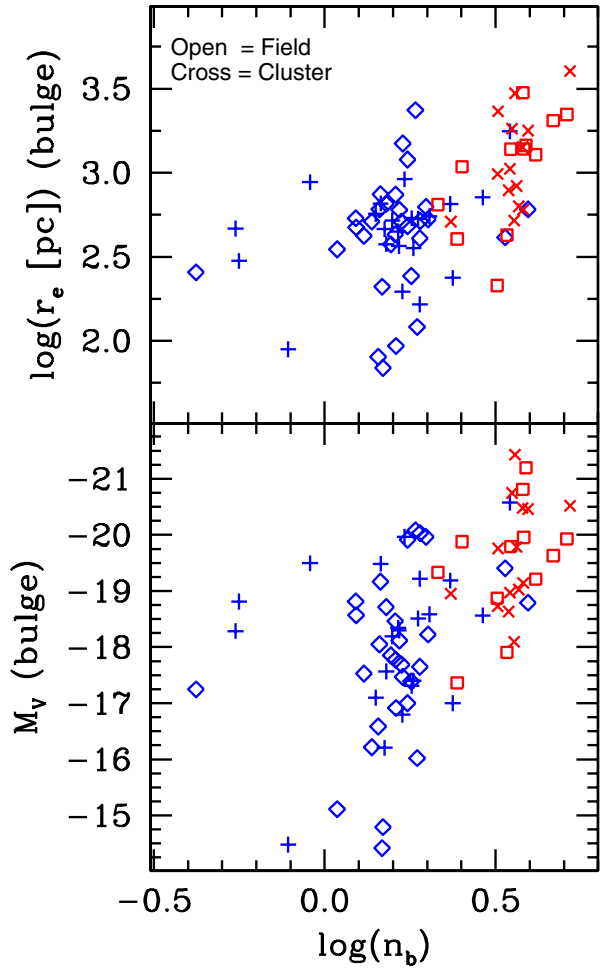

Figure 13. (a) Structural parameters of bulges as a function of the environment. Field galaxies with classical bulges are marked by open squares, and field pseudobulges with open diamonds. Cluster galaxies with classical bulges are marked by 'plus' symbols, cluster pseudobulge galaxies by crosses. All classical bulges are marked by red symbols, and pseudobulges by blue symbols. (b) As in (a), we distinguish those galaxies that exist in cluster (or group) environments (crossed symbols) from those that exist in the field (open symbols). Symbol color reflects bulge type, as before. The bulge-to-total ratio is plotted against absolute magnitude of the bulge. (c) Bulge-to-total ratio (lower panel) and ratio of the bulge half-light radius to the scale length of the disk (top panel) plotted against the logarithm of the bulge Sérsic index. As before, classical bulges are represented by open red circles and pseudobulges by blue crosses.

possible that pseudobulges in the field are formed through different mechanisms than those in cluster environments? We cannot know the answer to this for certain; however we can look for signatures for such effects in our data.

In Figure 13 we show our primary result again, namely that pseudobulges have a lower Sérsic index than classical bulges, but here we also mark environment. Field galaxies with classical bulges are marked by open squares, field pseudobulges with open diamonds. Cluster galaxies with classical bulges are marked by 'plus' symbols and cluster pseudobulge galaxies by crosses. There seems to be no significant differences in the structure of the surface brightness profile between the pseudobulges that reside in the field galaxies and those in cluster galaxies. Cluster pseudobulges are not higher mass nor systematically different in radial size than field pseudobulges. Nor are their surface brightness profiles preferentially steeper in clusters.

The same holds for $B / T$ : there is no substantial difference between pseudobulges in the field versus clustered environments. If pseudobulge formation was driven primarily by tidal encounters with distant galaxies, one would expect that this effect should be enhanced in cluster environments, where such encounters are more frequent. This would result in more massive pseudobulges existing in cluster environments. Our sample does not seem to indicate that pseudobulges are any more luminous in cluster environments than in the field. We feel that this supports
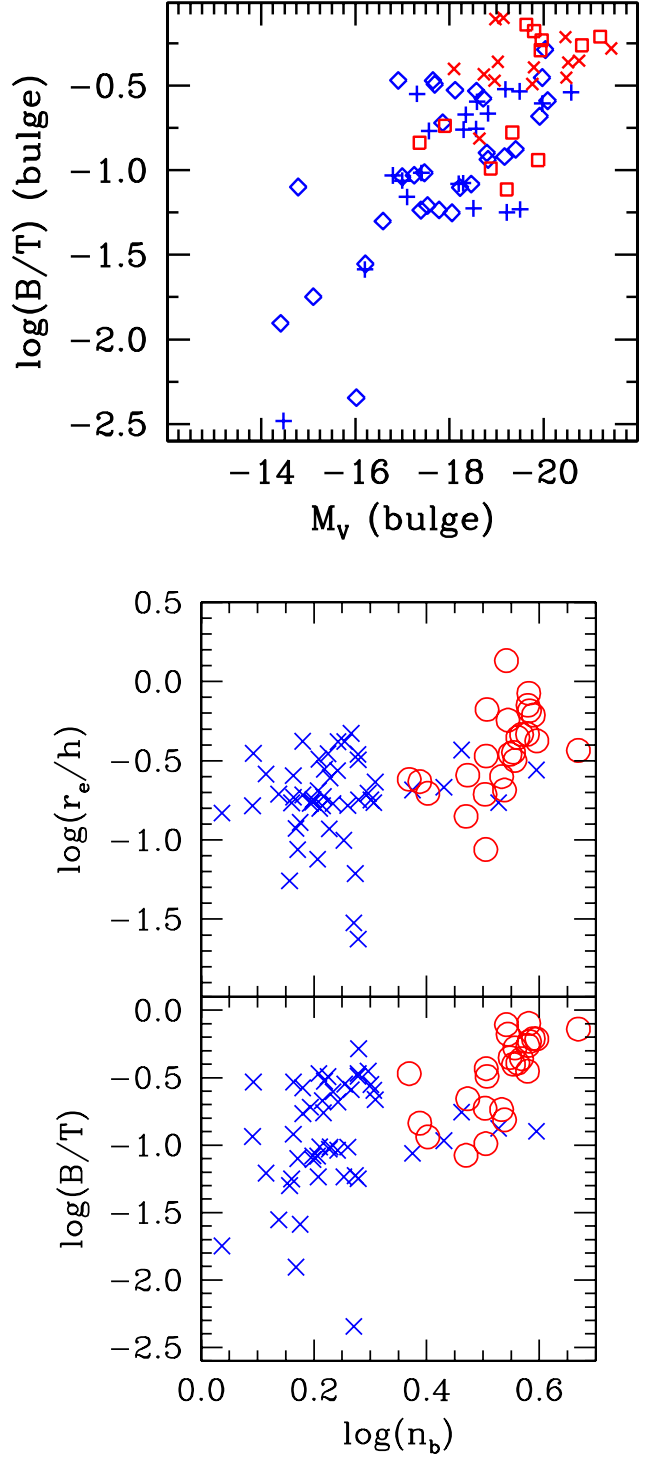

Figure 13. (Continued)

the notion that externally driven disk evolution is not likely the dominant effect in driving pseudobulge formation.

Our results in no way rule out the possibility that pseudobulges are formed by gas-rich minor mergers. If we take an example of merging our Galaxy with one of the Magellanic Clouds, and if this is done $\sim 1 \mathrm{Gyr}$, when the gas fractions were much higher, it is entirely plausible that the result could look similar to what we call a pseudobulge. However, it is not certain if such a system would still be actively forming stars today, or if pseudobulge formation through minor mergers could only happen in extreme cases (e.g., prograde collisions at low inclination). Also, it is not clear how such accretion-driven formation of pseudobulges could maintain the bulge-disk correlations discussed in the previous sections.

\section{SUMMARY AND DISCUSSION}

The main result of this paper is that bulge Sérsic index, $n_{b}$, is bimodally distributed in intermediate-type galaxies where both classical bulges and pseudobulges exist. A value of $n_{b} \simeq 2$ marks the boundary for separating morphologically-identified pseudobulges from classical bulges. Below $n_{b}=2$ no classical bulges are found, and above it very few pseudobulges are found. 
We also find that galaxies which are identified as pseudobulges, using either bulge morphology or bulge Sérsic index, are flatter than classical bulges. Thus suggesting that on average these systems are more disk like in both their morphology and shape than are classical bulges. Pseudobulges exist that are as round as classical bulges, yet, invariably the flattest bulges are all pseudobulges. In both Sérsic index and flattening ratio, our results show a homogeneity in classical bulge properties and a greater dispersion in pseudobulge properties. That is to say, classical bulges, in our sample, do not have Sérsic index less than 2, nor do they have high ratios of bulge-to-disk ellipticity. Pseudobulges, on average, have lower $B / T$ than classical bulges; however we find pseudobulges with $B / T$ extending to $\sim 0.35$.

We find that the half-light radius of pseudobulges is well correlated with the scale length of the outer disk, while the scale length of classical bulges is not correlated with that of the disk. This is consistent with the interpretation that $r_{e} \propto h$ is due to a secular formation of pseudobulges. Also, the fact that the scale length of the disk is correlated with the size of its pseudobulge but not correlated with the size of classical bulges is a strong indication that there indeed is a physical difference between the formation mechanisms of these different bulge types.

In photometric fundamental plane projections, pseudobulges populate and extend the low-luminosity end of the range occupied by classical bulges and elliptical galaxies. Pseudobulges deviate more from the relations set by the elliptical galaxies than classical bulges, and preferably toward lower density. In fact, it does not seem that, on their own, pseudobulges would establish any of these correlations, especially $M_{V}-\mu_{e}$.

In all correlations investigated in this work we find that the fundamental distinction is between classical bulges and pseudobulges. We do not find significant differences within the class of morphologies we identify as pseudobulges (nuclear spirals, nuclear bars, nuclear rings, and chaotic nuclear patchiness).

Is it possible that identifying pseudobulges visually using nuclear morphology is subject to an inherent flaw? That is, that these systems are by definition bulge-disk systems, and thus it is possible that classical bulges coexist over large radius with the central parts of a disk. Pseudobulges in S0 galaxies exemplify this concern. In many ways, elliptical galaxies and S0s are thought to be the extremes of a continuum of properties. Embedded disks are known to exist in elliptical galaxies (see, e.g., Scorza \& Bender 1995). Would we call such a system where the embedded disk had a nuclear bar a pseudobulge? Most likely not. However, in S0s, which by definition have lower $B / T$, we are inclined to do so. Clearly, if there is no sharp change in properties along the E-S0 continuum, there should be some composite systems with secularly formed structure in the disk and a classical (i.e., kinematically hot) bulge. (Note, though, that many S0s resemble defunct later-type disk galaxies much more than they do resemble elliptical galaxies as suggested by van den Bergh 1976.) This may be the case in NGC 2950, yet it does not appear to be the rule. Erwin et al. (2004) show that many bulges have more complicated dynamical profiles, with both hot and cold components. Yet, pseudobulges show a remarkable similarity to disk stellar populations, ISM, and star-formation rates. These similarities have been shown in the flatness of stellar population gradients (Peletier \& Balcells 1996) and the similarity of $\mathrm{CO}$ profiles to optical light (Regan et al. 2001). Helfer et al. (2003) show that many CO gas maps of bulge-disk galaxies have holes in their center, but also many $\mathrm{CO}$ profiles rise steeply to their center. A quick comparison with our sample shows that those holes are found in classical bulges (e.g., NGC 2841). Fisher (2006) shows that the nuclear morphology of galaxies (as used here) predicts the shape of the 3.6-8.0 $\mu \mathrm{m}$ color profile: disk-like bulges have flat color profiles and E-like bulges have holes in $8.0 \mu \mathrm{m}$ emission. If we were merely mistakenly identifying disks superimposed on bulges as physically different pseudobulges, we would expect to find larger $B / T$ for such systems as compared to the bulges where we do not see evidence for the presence of a disk which we call classical in this work. This is because of the added light of the disk onto a fraction of bulges drawn from the same underlying distribution. However, we do not find this to be the case. Figure 6 shows that pseudobulges are on average smaller than classical bulges.

It is not clear whether pseudobulges participate in the various correlations presented in this paper. If we look at the pseudobulges alone, they only show a convincing correlation in the Kormendy relation $\left(\mu_{e}-r_{e}\right)$. Their distribution better resembles scatter diagrams in the $M_{v}-\mu_{e}, \mu_{e}-n$, and $r_{e}-n$ planes. However, it would seem to be an odd coincidence that in every parameter combination pseudobulges just happen to fall in the right location to extend the correlations set by the classical bulges and the elliptical galaxies. Putting all this together implies that we do not really understand the details of pseudobulge formation very well. It is also possible that the decomposition of pseudobulges may not be an appropriate procedure. Bulge-disk decomposition assumes that the structures are distinct (and that light extends to radius of infinity). If we accept that pseudobulges are formed through disk phenomena, then such a bulge-disk decomposition may not be an adequate description of those systems. In reality they might simply be a component of galactic disk. Also, parameters derived from the Sérsic function are coupled (Graham \& Colless 1997). Thus, treating pseudobulges in the same way as classical bulges may artificially force them to extend some photometric correlations.

Concerning the six bulges with disk-like morphology near their centers that have $n_{b}>2$, two have $\left\langle\epsilon_{b}\right\rangle /\left\langle\epsilon_{d}\right\rangle>0.75$ (the maximum of classical bulges), and NGC 4314 has a very prominent star-forming nuclear ring, making this bulge a strong candidate for being a pseudobulge. This galaxy might be an example of a composite system. Their existence certainly underscores the value of having as much information as possible when diagnosing bulge types. Future work may prove illuminating. For example, to what extent should we trust bulge morphology as an indicator of secular evolution? It is possible for the human eye to mistake merely the presence of dust for spiral structure?

We also find that the ratio of scale lengths, $r_{e} / h$, in pseudobulges is more tightly correlated and closer to those values reported by other authors (e.g., MacArthur et al. 2003) than in classical bulges. However, the range in values of $r_{e} / h$ for classical bulges and pseudobulges is similar; therefore, this does not provide a good diagnostic tool for finding pseudobulges. Pseudobulges extend the parameter correlations of photometric quantities $\left(r_{e}, \mu_{e}, M_{V}\right.$, and $\left.n_{b}\right)$. However, in many of these parameters it is unclear if pseudobulges actually show a correlation on their own.

We can compare the structural properties of the bulges in our sample with the output of simulation. Unfortunately simulations of galaxies that resemble real galaxies including stars, dark matter, gas and star formation (and possibly feedback) are quite difficult. Thus, there does not exist a statistically relevant set of 
simulations for full comparison. Nonetheless, we can compare our results to those that currently exist.

Debattista et al. (2004) provide a set of simulations that generate bulges from bar buckling in pure stellar (no gas) systems. The resulting bulges typically have $n_{b} \sim 1.5$, which is consistent with what we find. The bulges in our sample tend to be more round than their associated outer disk, and Debattista et al. (2004) separate their bulges based on flattening ratios. So we will only compare to those bulges that are less round than the outer disk. Their simulated galaxies have $B / D=0.2-1.0$ (where $B / D$ is the bulge-to-disk light ratio). Whereas our sample has a median $B / D=0.12$ with a standard deviation of 0.20 . Further, they are able to recover the coupling of $r_{e}$ and $h$ that we find in pseudobulges. Thus our pseudobulges tend to be a bit smaller. It may be that bar buckling is one way to make a pseudobulge, as indicated by the fact that those simulated bulges from Debattista et al. (2004) are contained within the set of pseudobulges, but do not span the whole range in properties.

We reiterate a statement by Andredakis (1998), that is also discussed in Kormendy \& Kennicutt (2004), namely that we do not really understand why pseudobulges should have a certain Sérsic index. It is understandable that mergers would drive up the Sérsic index, as discussed by van Albada (1982) and Kormendy et al. (2008), and thus classical bulges are found with higher Sérsic index. Aguerri et al. (2001) simulate minor mergers (accretion of satellites on the order of the mass of the bulge) in bulge-disk galaxies. They show that the Sérsic index grows as the amount of mass accreted becomes larger. Eliche-Moral et al. (2006) take the study of satellite accretion to lower densities than those simulations of Aguerri et al. (2001). Their simulations do not include gas and star formation, and thus as with the Debattista et al. (2004) simulations they should be read with that caveat in mind. They find that low-density satellite accretion does not necessarily drive Sérsic index above the critical value we find $\left(n_{\text {crit }} \sim 2\right)$. Yet, they also find that satellite accretion leads to a simultaneous increase in $B / T$.

In Figure 13(c), we therefore plot the bulge-to-total ratio, $B / T$, and the ratio of the bulge half-light radius to the scale length of the disk, $r_{e} / h$, against $n_{b}$. There is a tight correlation for classical bulges. However, pseudobulges do not follow this correlation. In fact, neither $B / T$ nor $r_{e} / h$ correlates with $n_{b}$ in pseudobulges at all in the range of parameters shown, spanning an order of magnitude in $B / T$. The absence of correlation between $n_{b}$ and $B / T$ in pseudobulges seems to be pointing to a nonmerger-driven formation scenario for pseudobulges. Yet, these results are suggestive at best. There may be some underlying correlation that is destroyed by other factors (e.g., gas fraction or collision parameters). More work is needed from both simulations and observations. That we do not see correlation of $n_{b}$ and $B / T$ for low-Sérsic index bulges (as found in Eliche-Moral et al. 2006) may be indicating that the classical bulges we observe today are the products of multiple mergers, and possibly at higher redshifts there is a population of lowSérsic index classical bulges. Conversely it is entirely possible that a population of small classical bulges exists, yet they are embedded within pseudobulges. In this case the mass of a bulge, and hence the $B / T$ within a specific galaxy would be coming from multiple mechanisms.

If pseudobulges are built by secular evolution, the simplest mechanism controlling the Sérsic index in bulges is that $n_{b}$ grows with time as the bulge-to-total ratio increases. However, as discussed above, it appears that there is not a strong connection between $B / T$ and Sérsic index. This would imply that Sérsic index in pseudobulges is not a time-dependent quantity. We wish to emphasize, though, that the error in the measurement of $n_{b}$ is large and might be masking an underlying weak correlation. Also, by focusing on intermediate-type galaxies, where both pseudobulges and classical bulges occur, we miss very low $B / T$ systems. Expanding a sample to later types might reveal a weak correlation (Graham 2001). Since $r_{e}$ correlates tightly with $h$ for pseudobulges, it is no surprise that $r_{e} / h$ does not correlate with $n_{b}$ in pseudobulges. The linear coupling of $r_{e}$ and $h$ is well established in late-type galaxies (Courteau et al. 1996).

It may be that the Sérsic index of pseudobulges is merely another manifestation of the dynamical state of the system. Stars in bulges with larger amounts of random motion often take on radial orbits, thus climbing higher out of the potential well. Thus there is more light at large radius, increasing $n_{b}$. However, stars in orbits with higher amounts of angular momentum would be less likely to take on radial orbits and thus bulge light would contribute less at large radius, driving $n_{b}$ down. The observation that the distribution of Sérsic indices in bulges of galaxies from $\mathrm{E}$ to $\mathrm{Sc}$ is bimodal then strengthens the claim that what we are calling pseudobulges are not merely the low-mass counterparts of the same phenomena that form classical bulges and elliptical galaxies. This description fits well with the theory that secular evolution forms pseudobulges, and mergers, whether by a single event or succession of events, form classical bulges. The higher angular momentum (and thus low-Sérsic index) systems have not had major mergers and thus have not experienced the violent processes that lower the ratio of rotational velocity to velocity dispersion. What is left unknown is why pseudobulges are not exponential, and also what keeps them from having larger Sérsic indices.

We conclude by returning to the results of Figure 6 and the implications of the range of $B / T$ for pseudobulges. It is now well known that the presence of many low- $B / T$ systems presents a problem for current $\Lambda \mathrm{CDM}$ galaxy formation theories (D'Onghia \& Burkert 2004). In our sample we find no classical bulge galaxies with $B / T<0.1$. Recent studies which compare merger histories in $\Lambda \mathrm{CDM}$ simulations to the observed frequency of bulgeless galaxies suggest that either there are too many mergers in the simulations or that disks must be much more robust to the merging process than previously thought (Stewart et al. 2007; Koda et al. 2007). Pseudobulges span a range of $B / T$ from 0.35 to 0 . If pseudobulges form through internal-disk processes then a galaxy with a pseudobulge can be thought of as a pure disk galaxy. Thus current estimates of the number of low- $B / T$ systems could only be thought of as lower limits; the existence of pseudobulges would make the problem of forming bulgeless systems even more pressing.

We would like to thank Professor John Kormendy \& Dr. Mark Cornell for countless discussions on this work. Also, D.B.F. would like to thank Professor Alex Filippenko and University of California at Berkeley for providing him with a place to do much of this research.

D.B.F. acknowledges support by the National Science Foundation under grant AST 06-07490. This work is based in part on observations made with the Spitzer Space Telescope, operated by the Jet Propulsion Laboratory, California Institute of Technology under a contract with NASA. Support for this work was provided by NASA through an award issued by JPL/Caltech.

This paper makes use of data obtained from the Isaac Newton Group Archive which is maintained as part of the CASU Astronomical Data Centre at the Institute of Astronomy, Cambridge. 

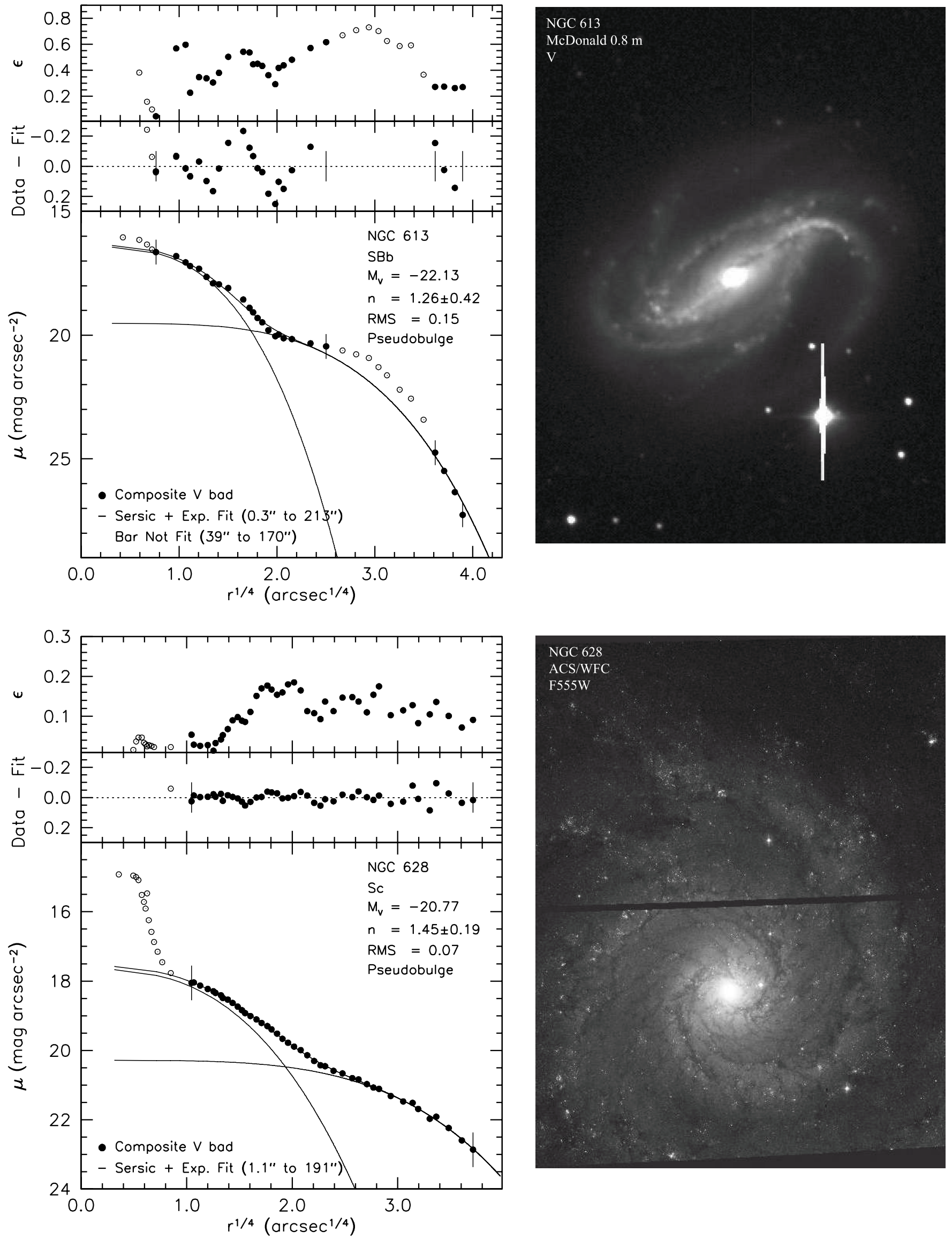

Figure 14. Profiles of all bugle-disk decompositions in this paper. Plot axes and symbols are the same as in Figure 4 . For reference we also show an image of the corresponding galaxy of each profile, so that features removed from the fit, such as bars, can be identified by the reader. 

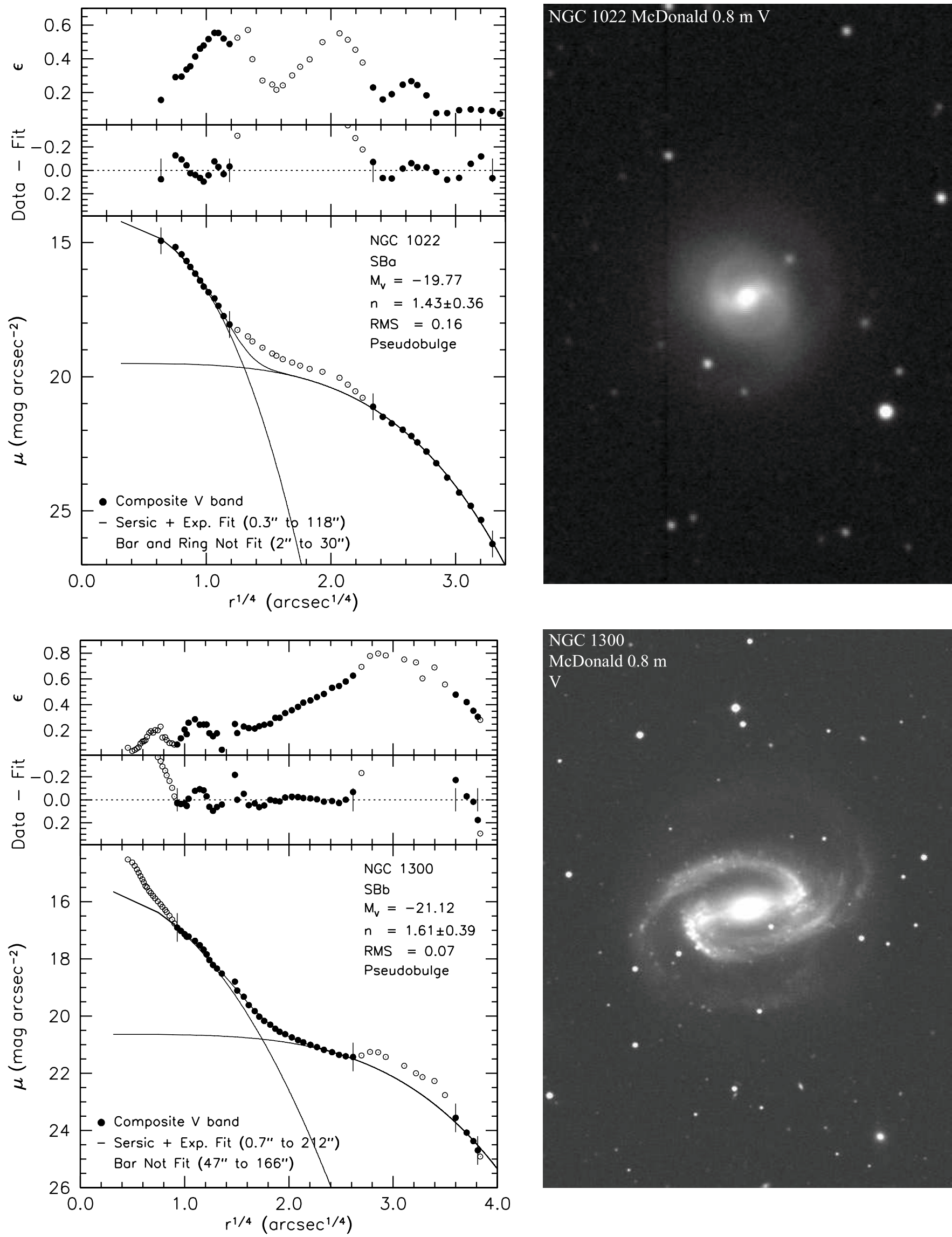

Figure 14. (Continued) 

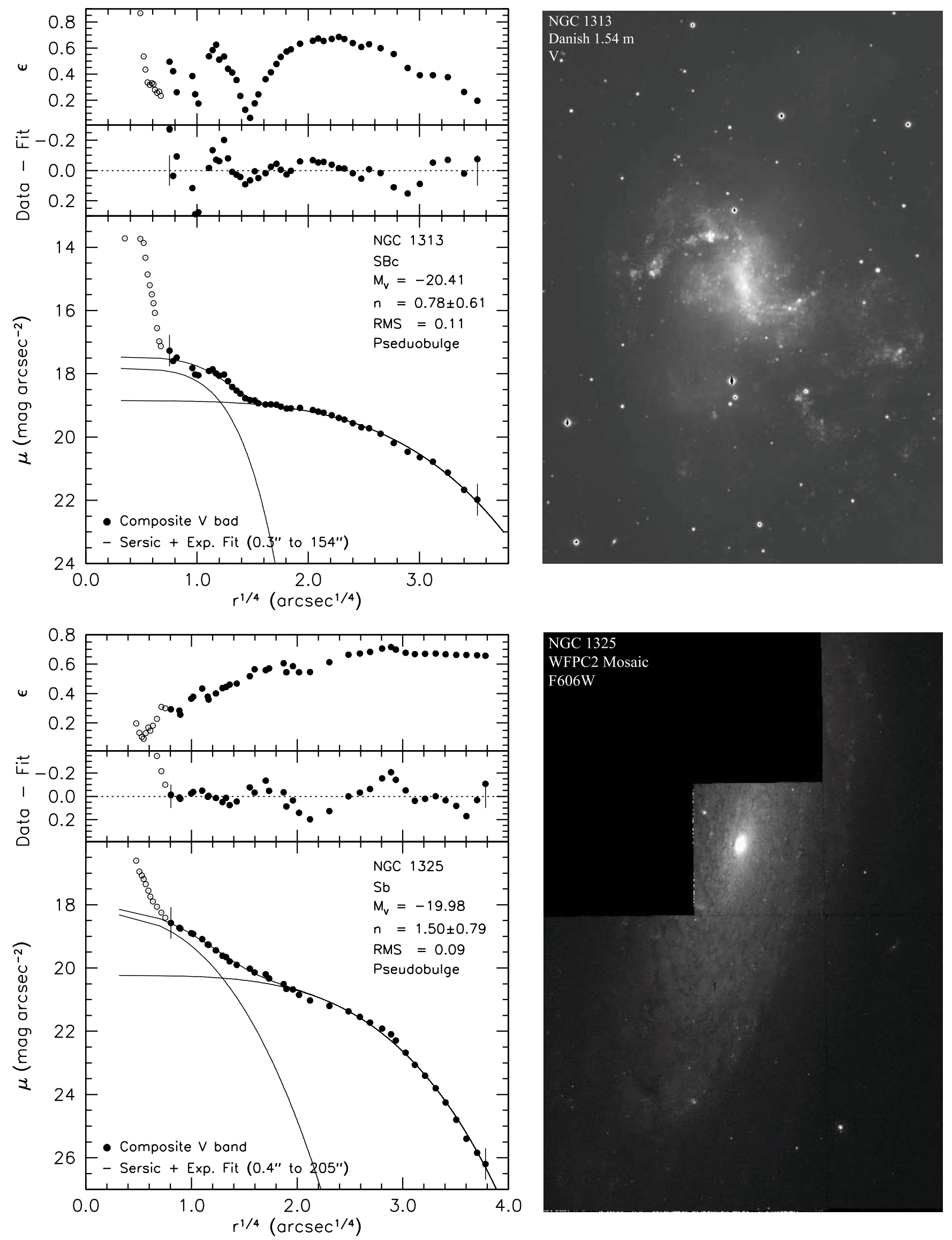

Figure 14. (Continued) 

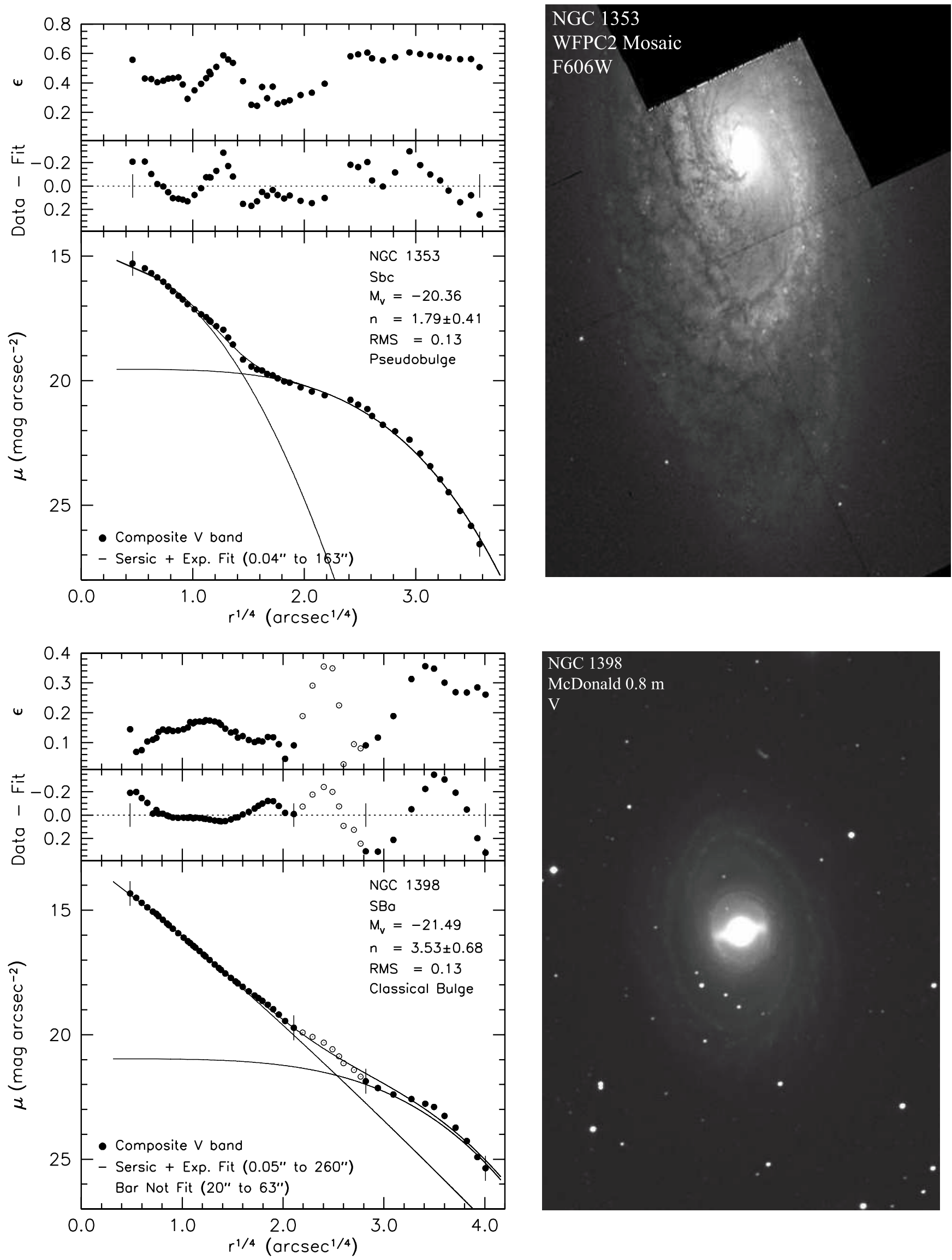

Figure 14. (Continued) 

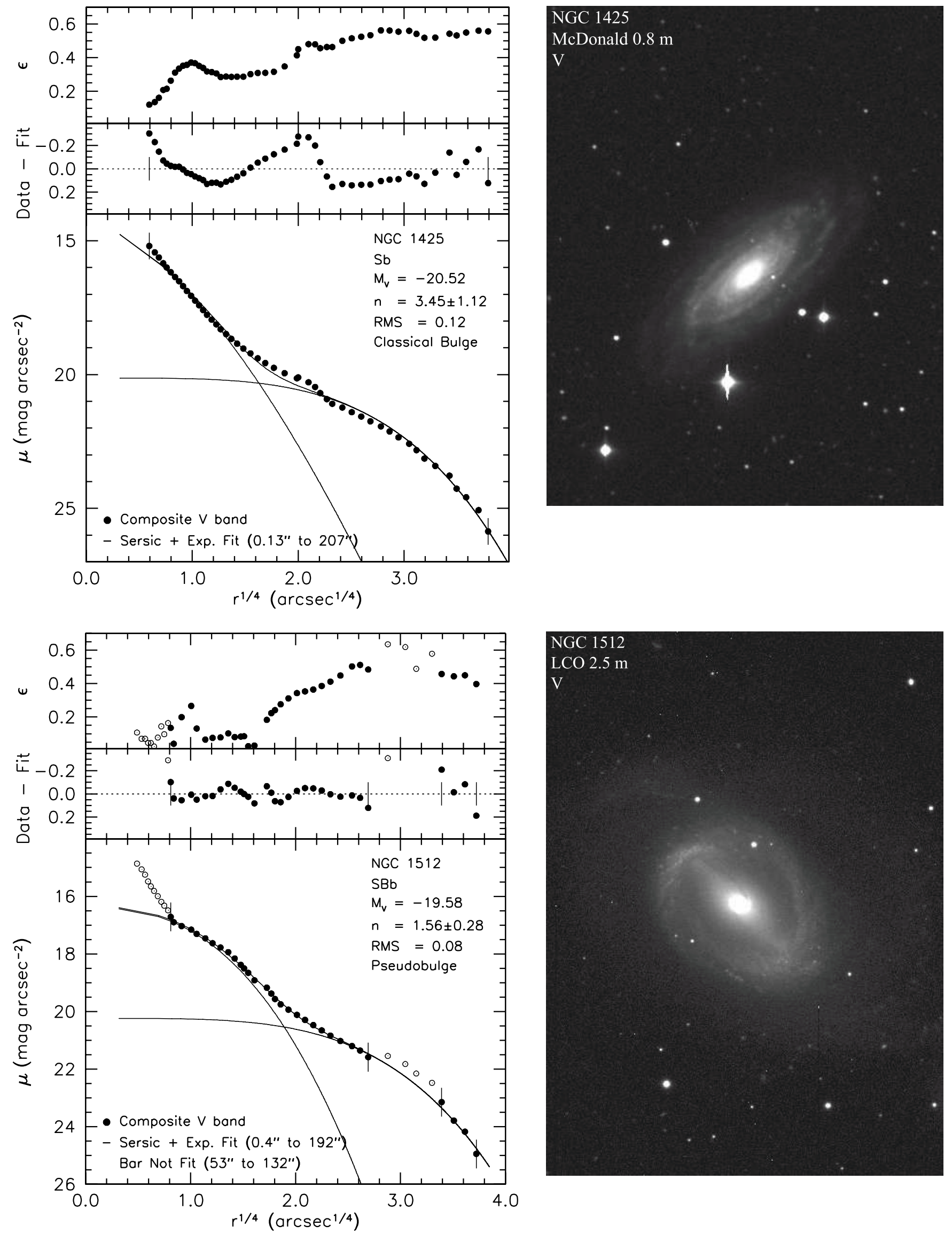

Figure 14. (Continued) 

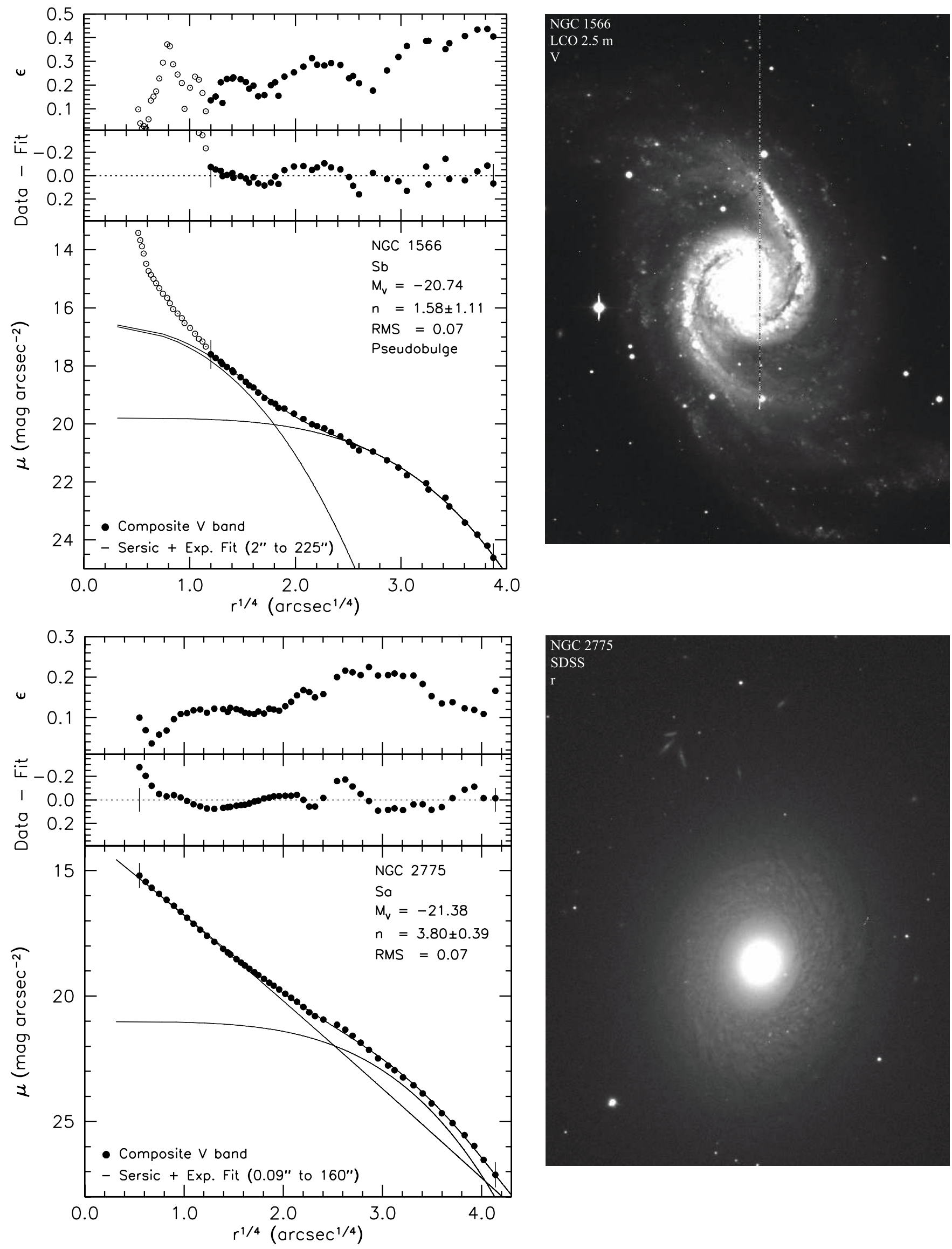

Figure 14. (Continued) 

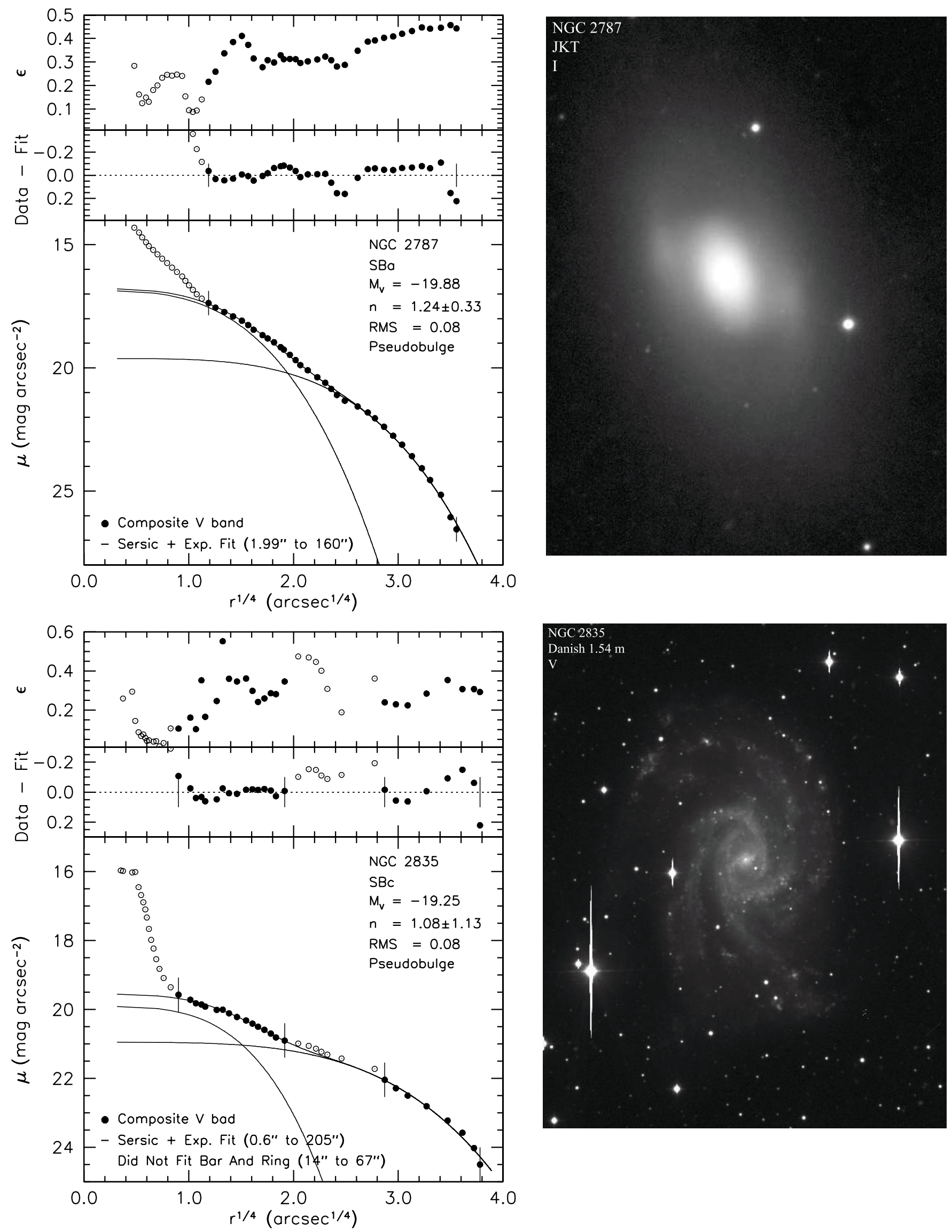

Figure 14. (Continued) 

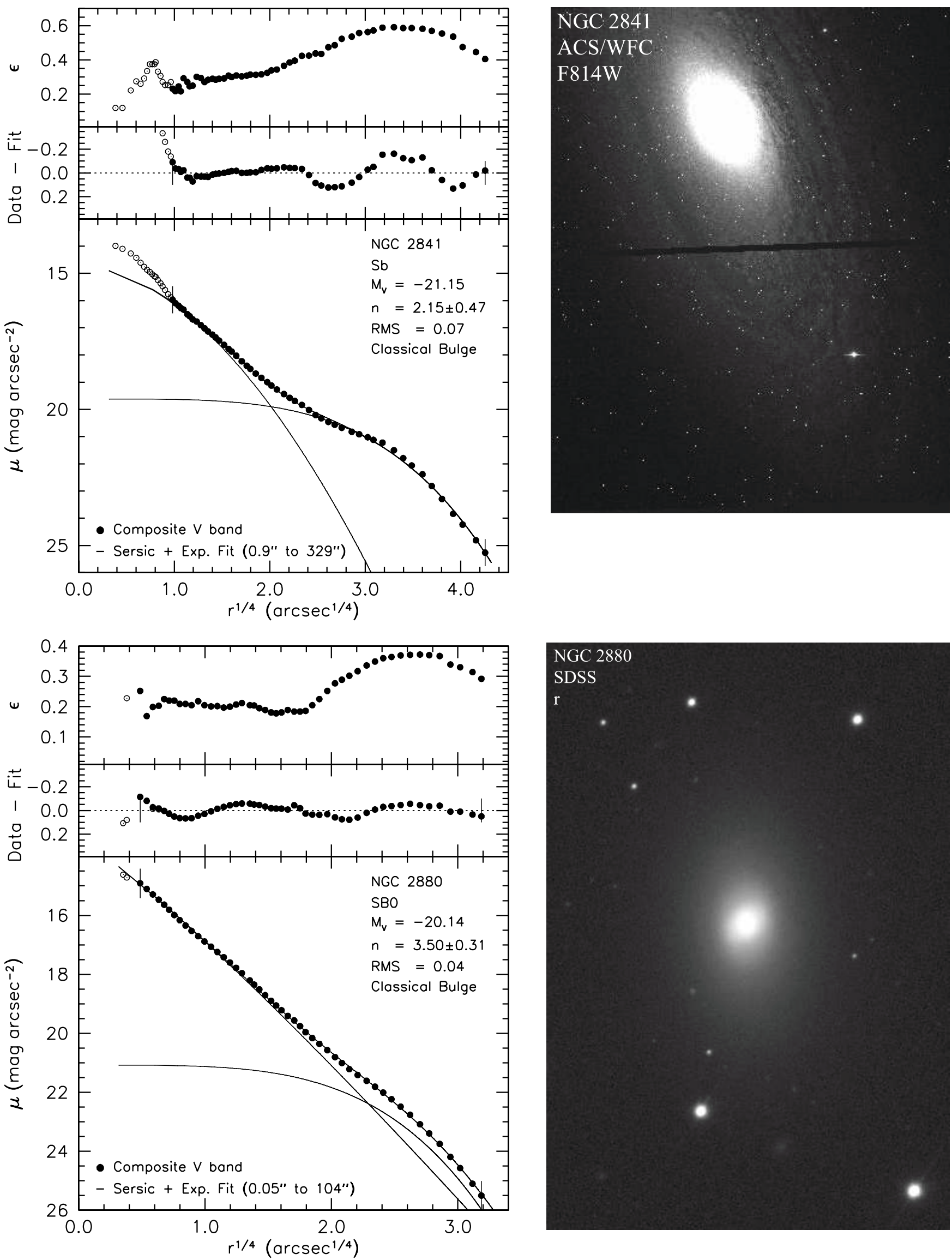

Figure 14. (Continued) 

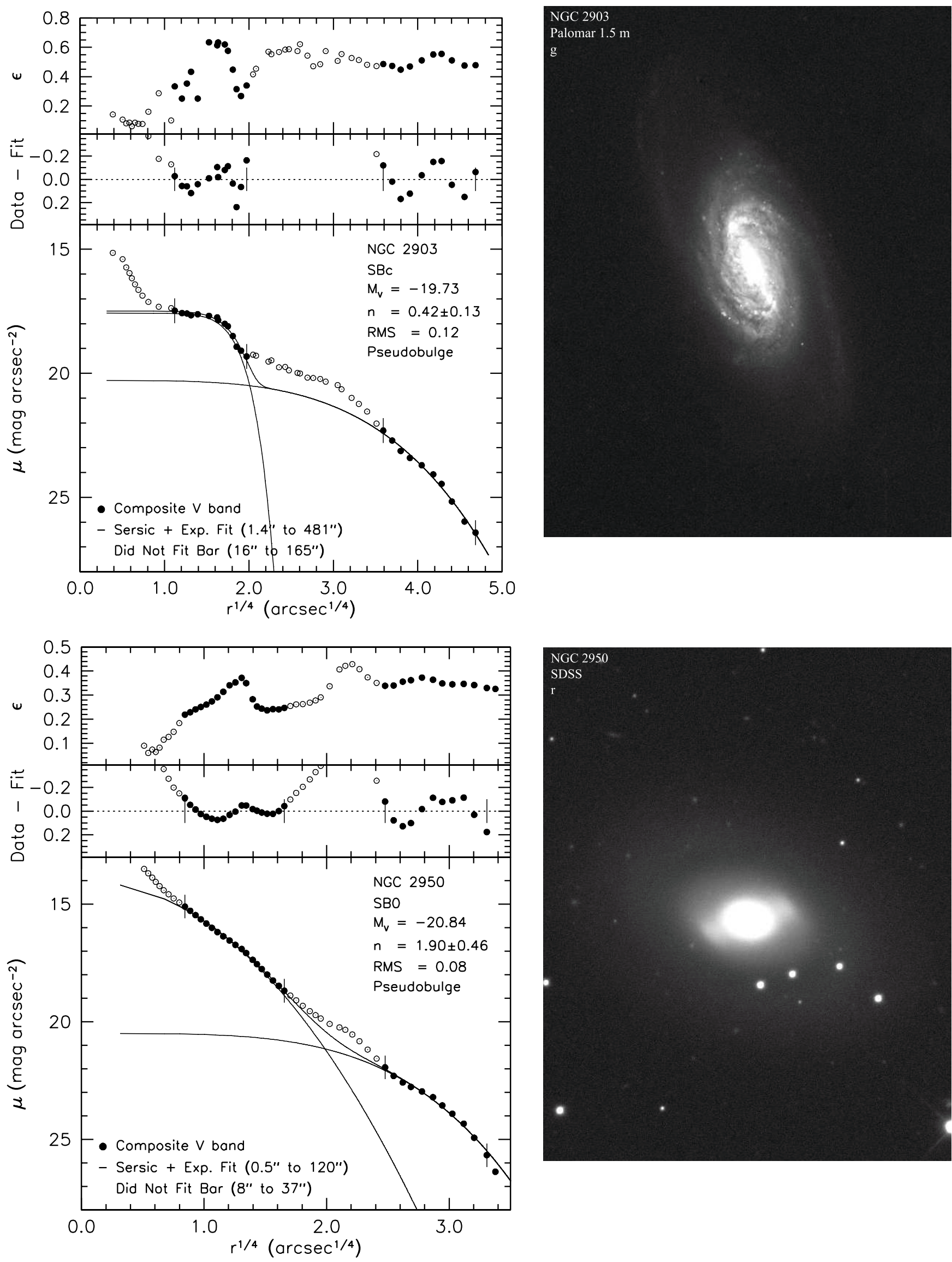

Figure 14. (Continued) 

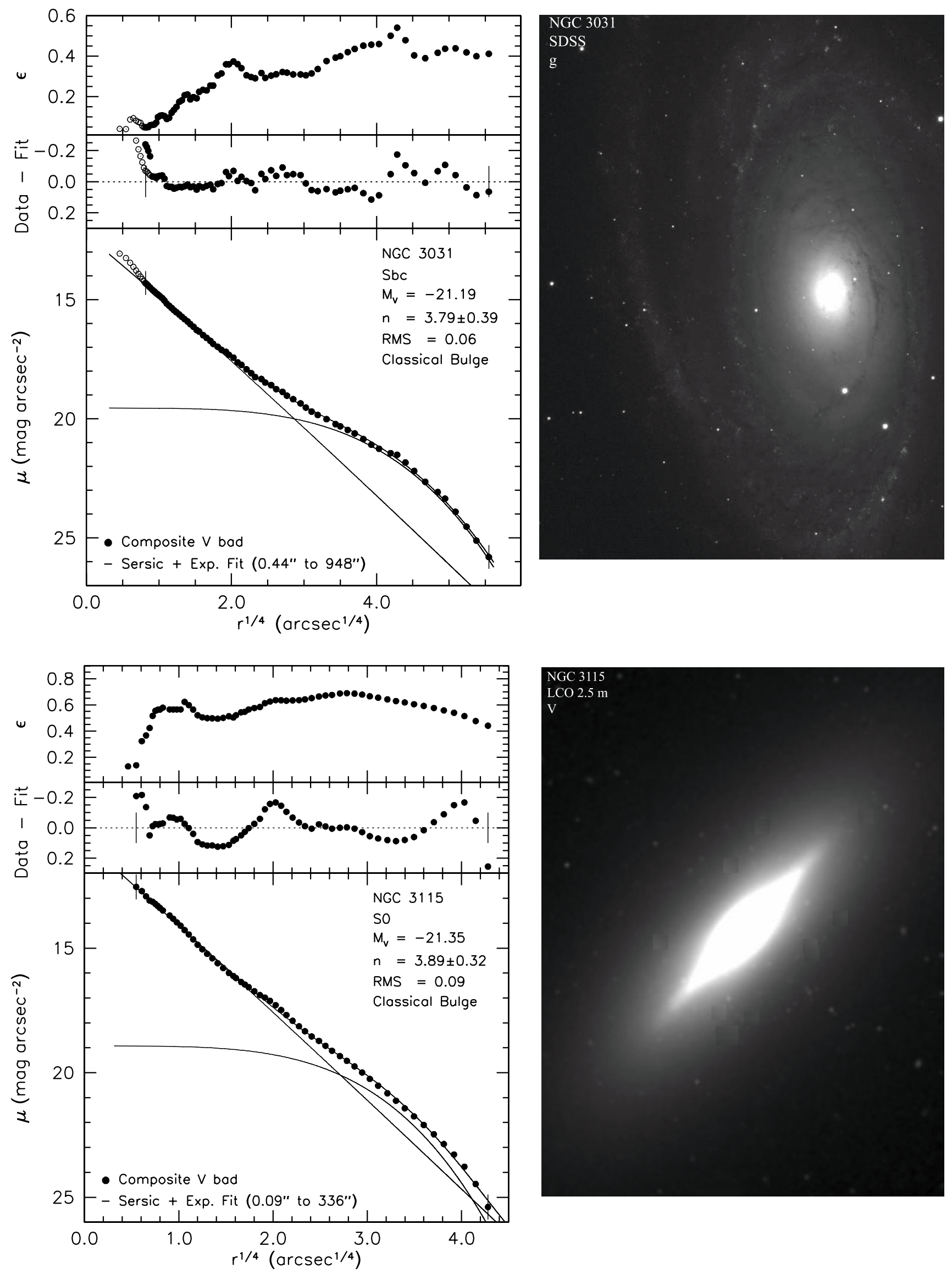

Figure 14. (Continued) 

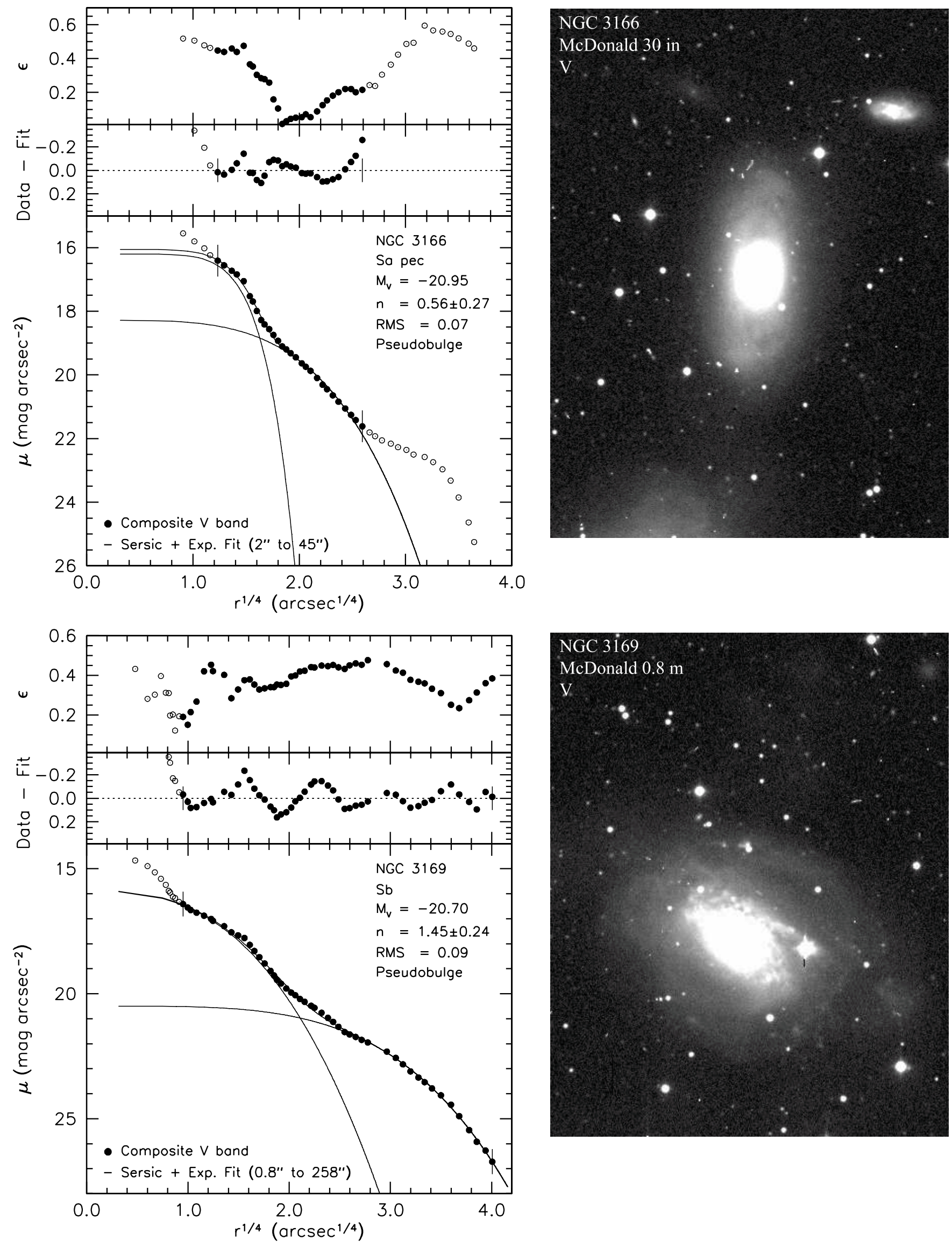

Figure 14. (Continued) 

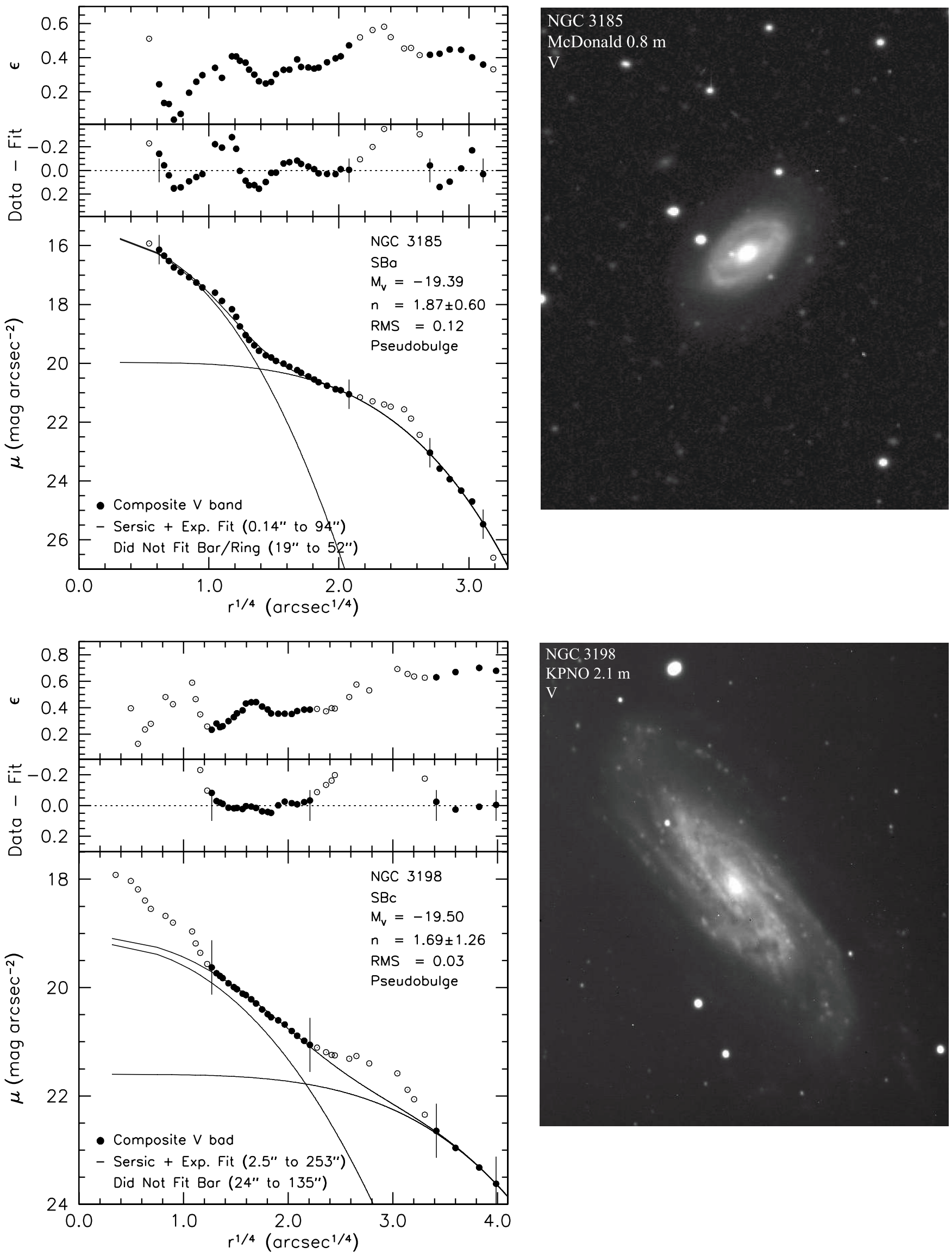

Figure 14. (Continued) 

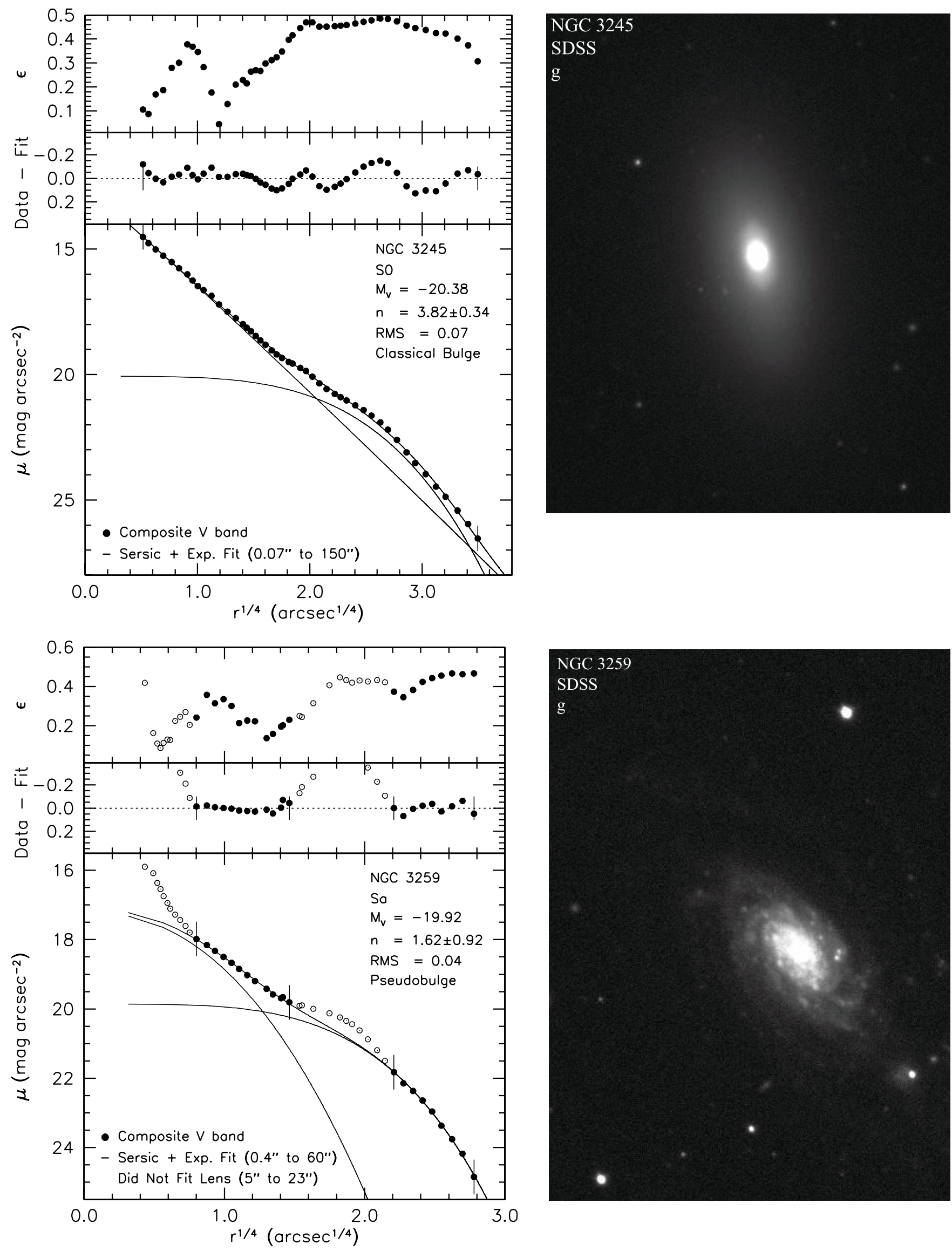

Figure 14. (Continued) 

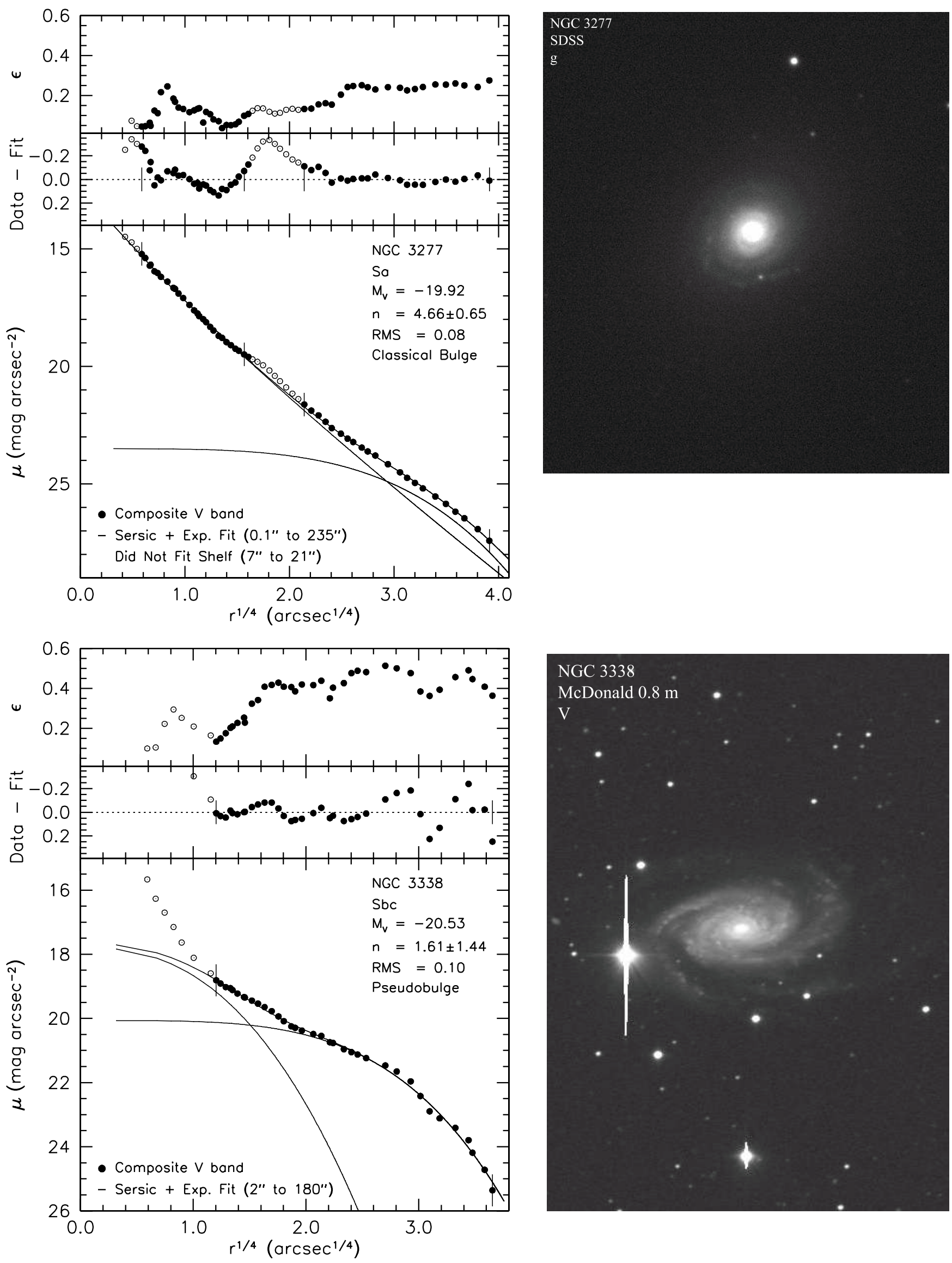

Figure 14. (Continued) 

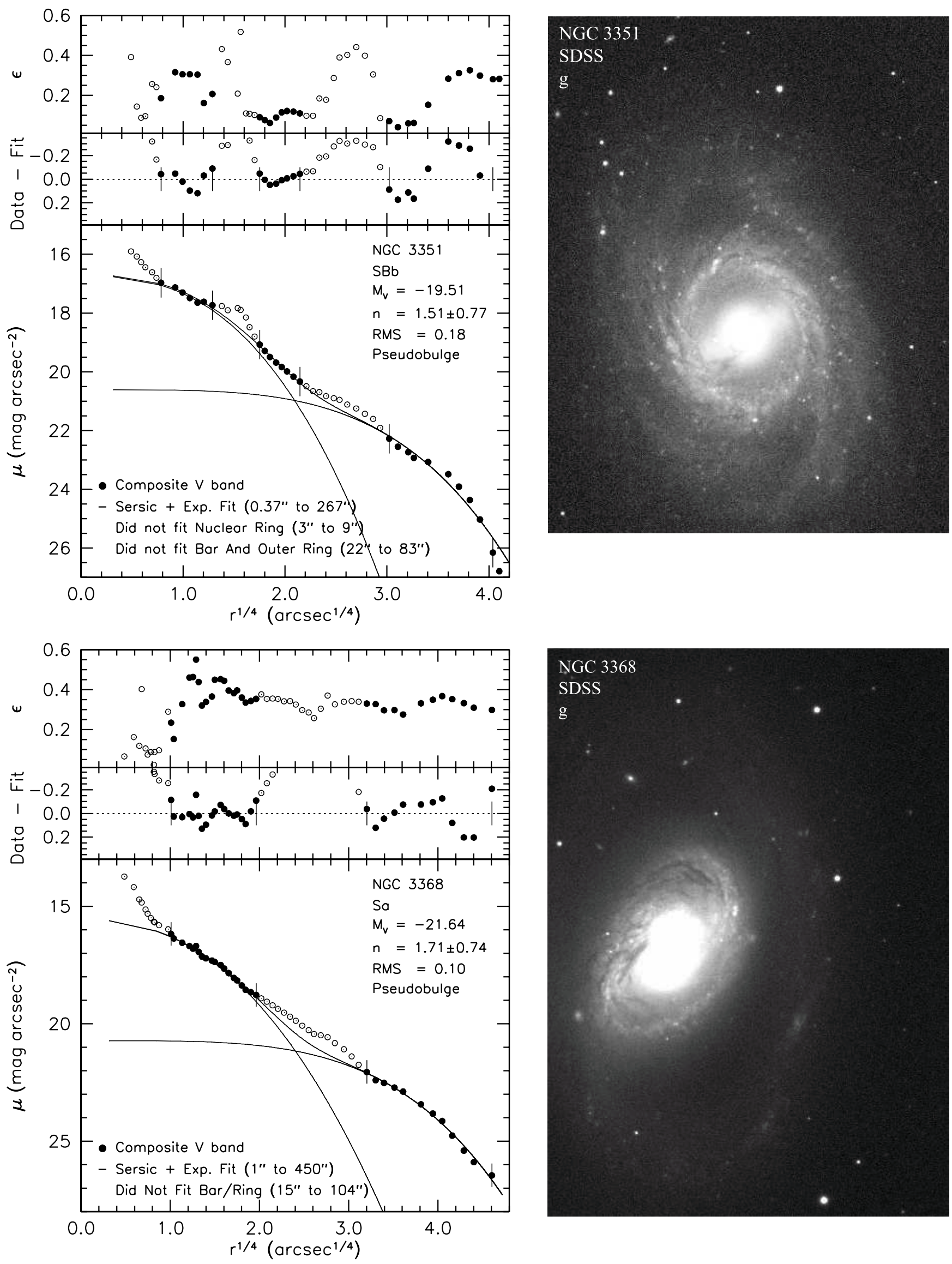

Figure 14. (Continued) 

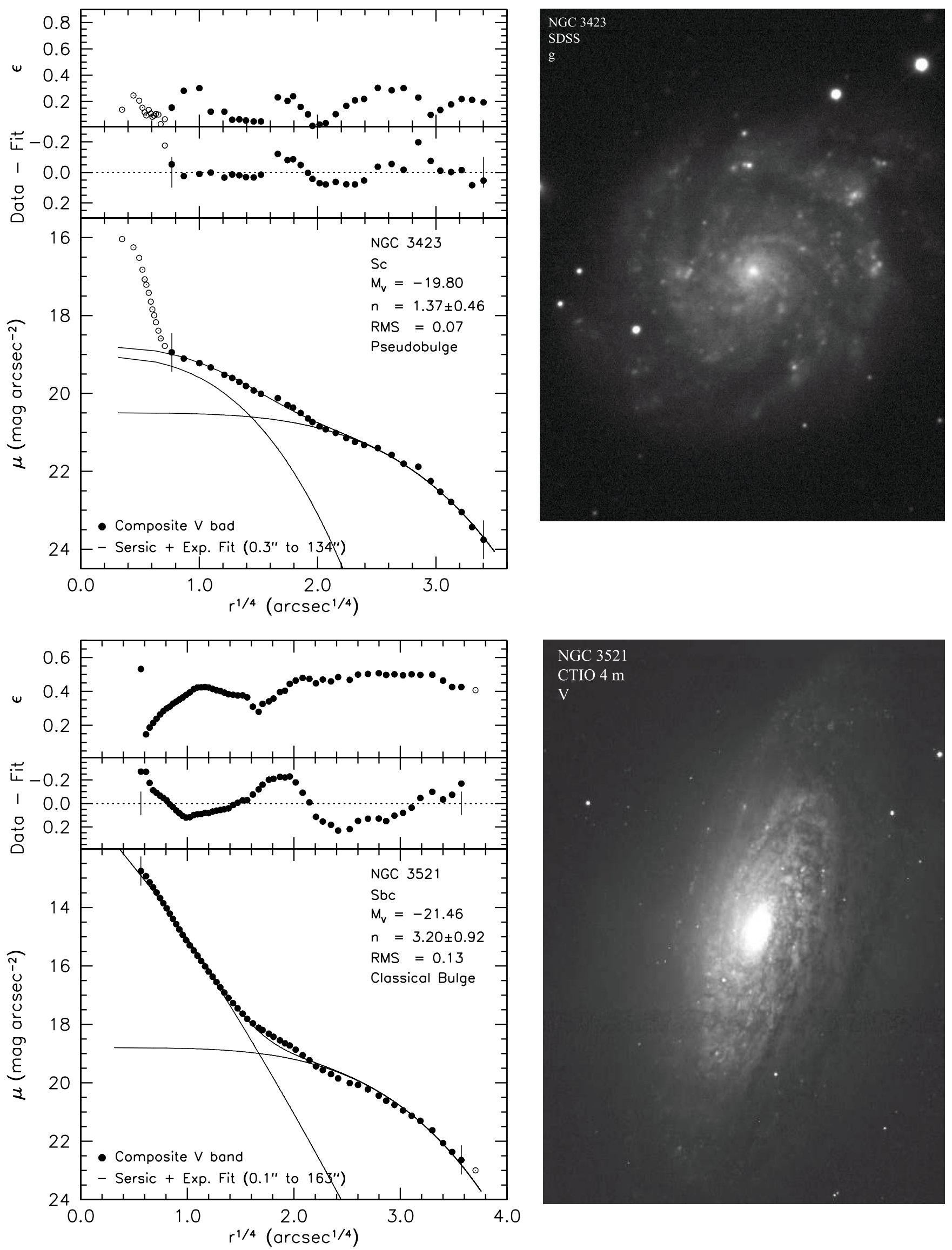

Figure 14. (Continued) 

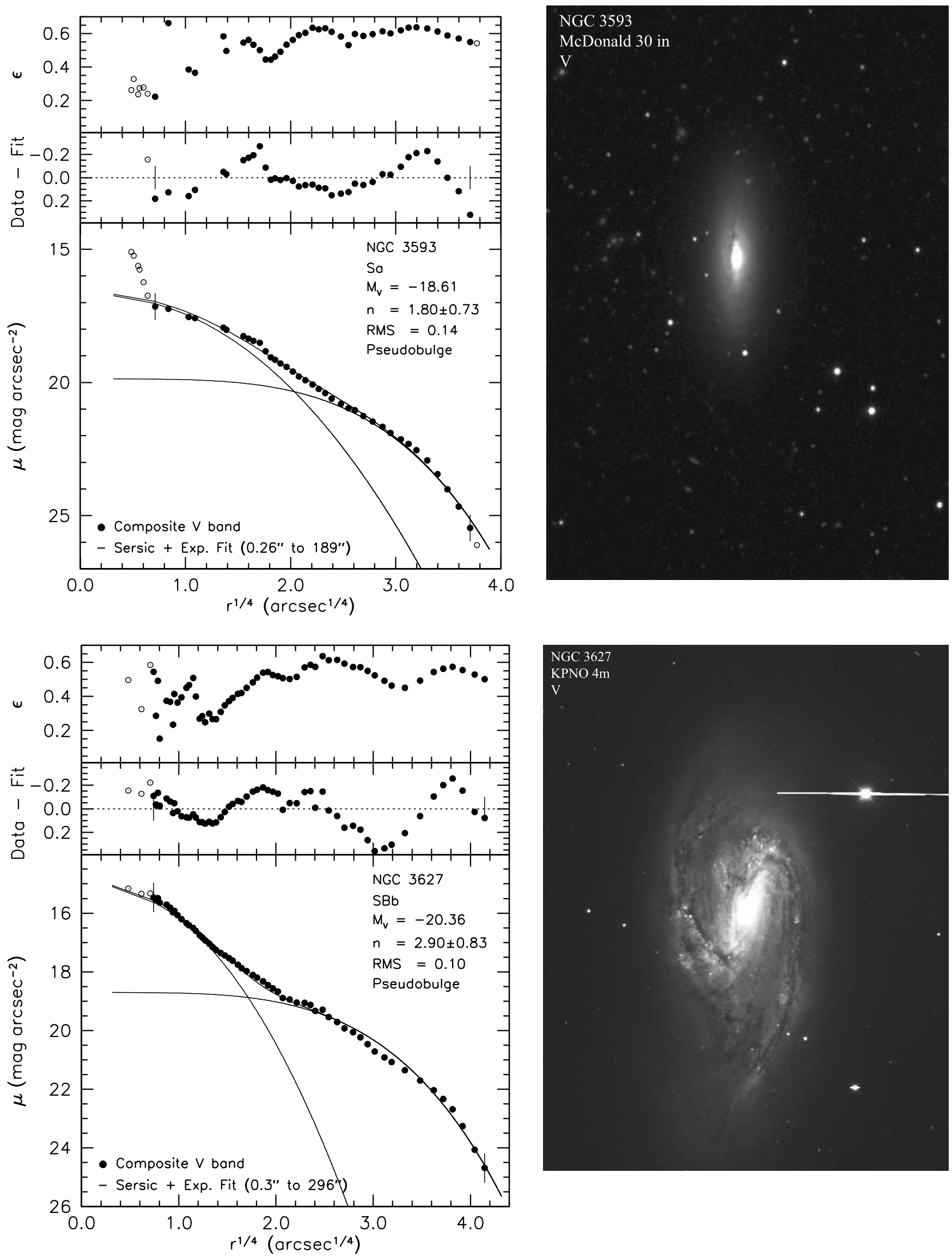

Figure 14. (Continued) 

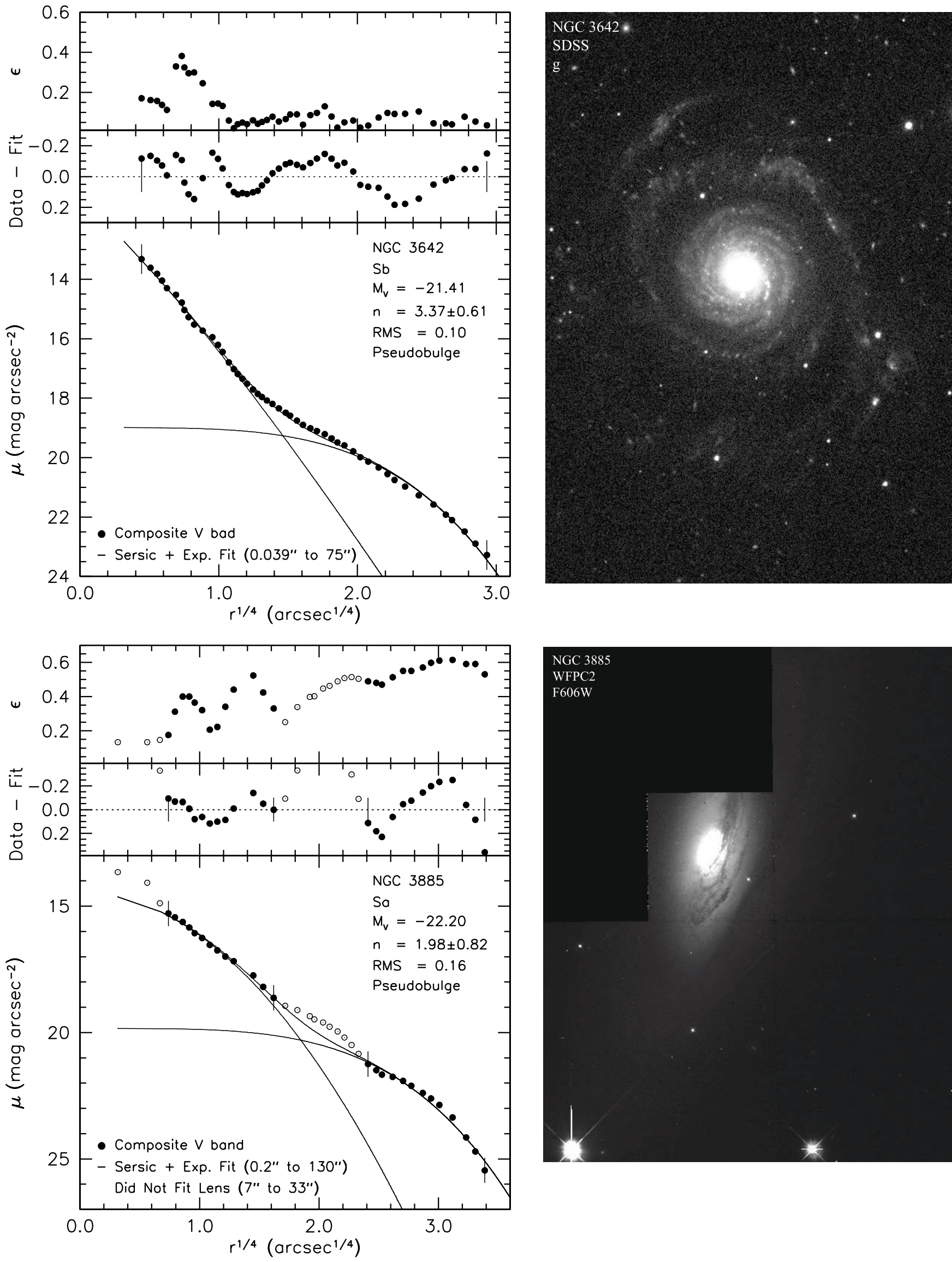

Figure 14. (Continued) 

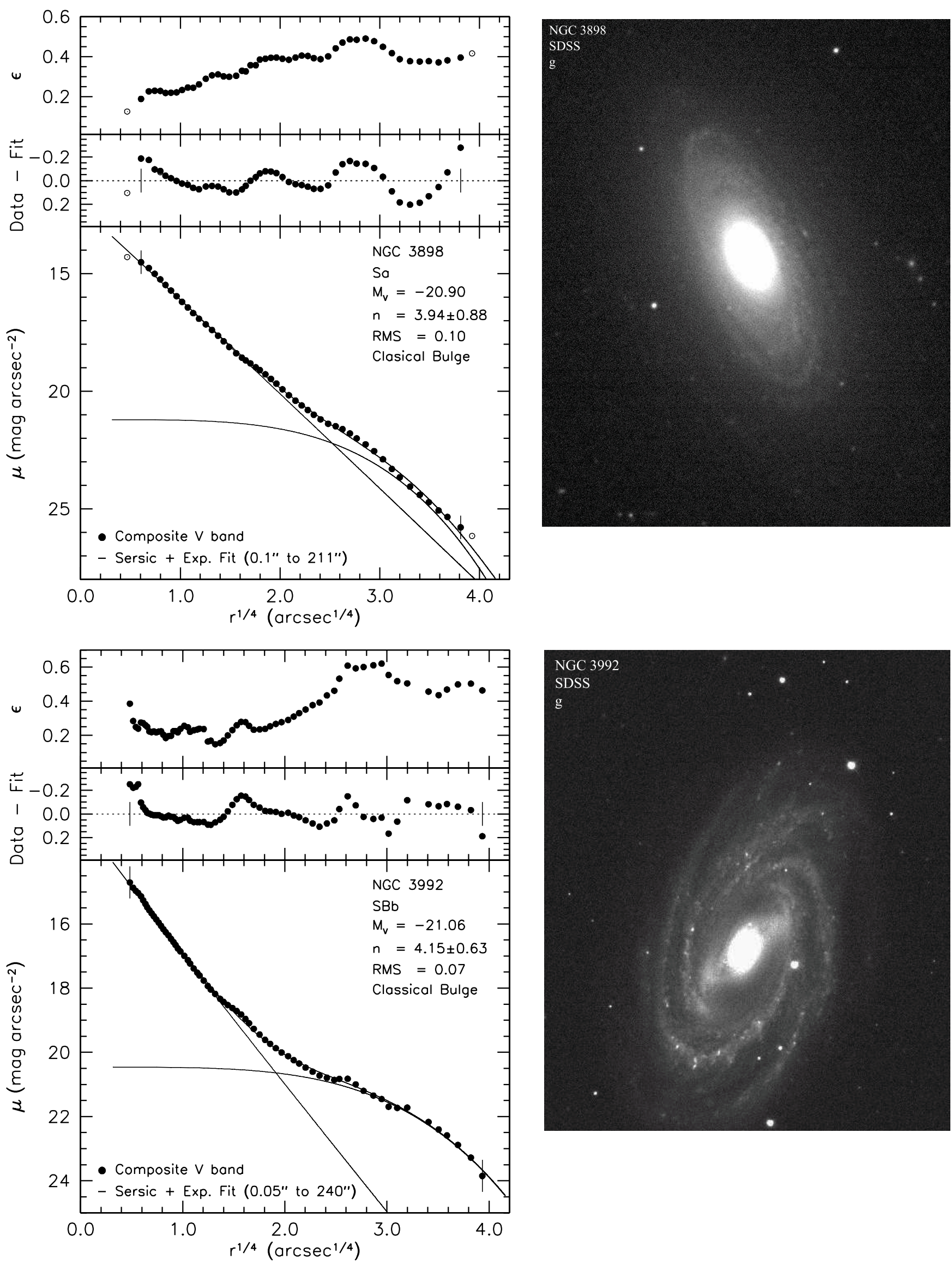

Figure 14. (Continued) 

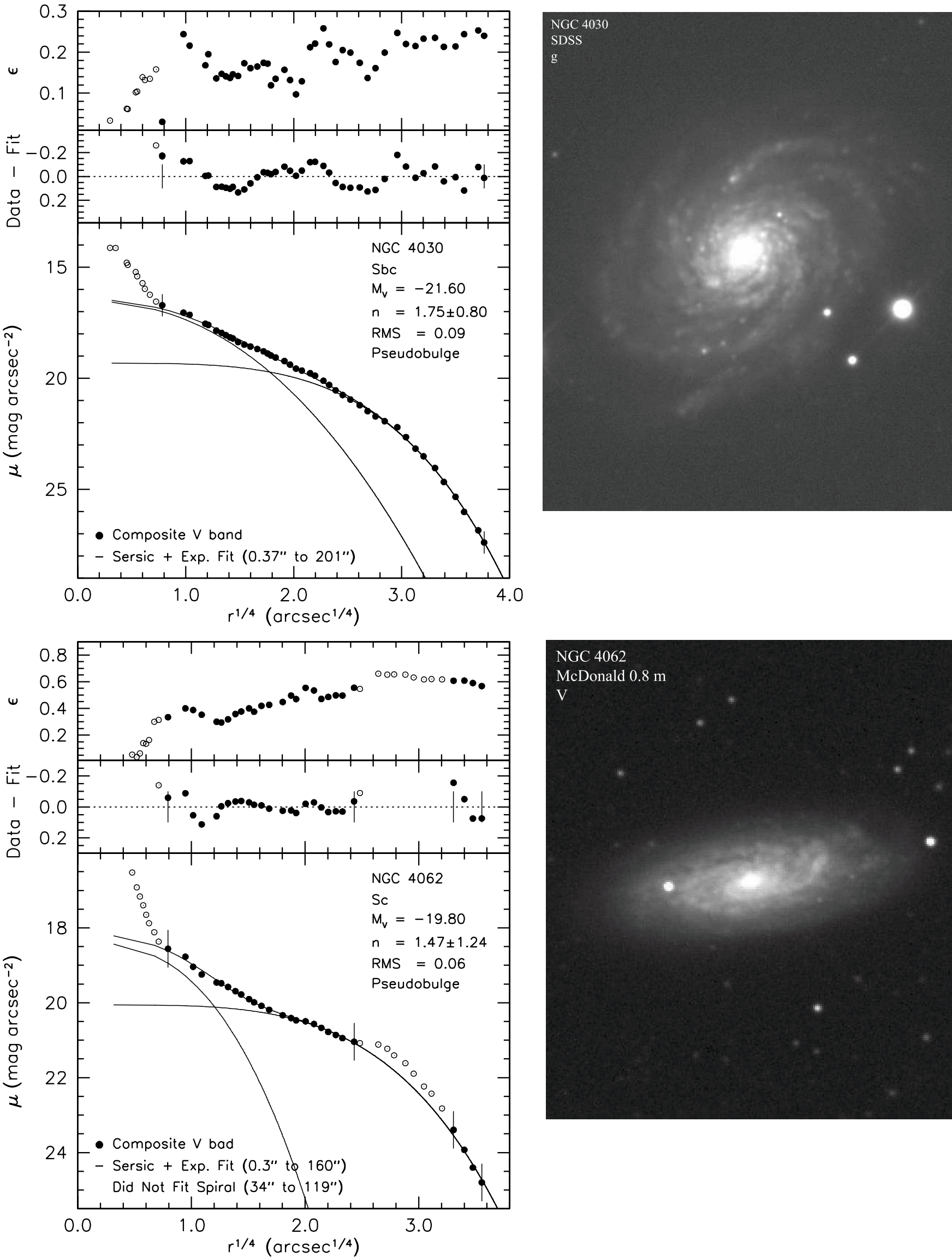

NGC 4062

McDonald $0.8 \mathrm{~m}$

$\mathrm{V}$

Figure 14. (Continued) 

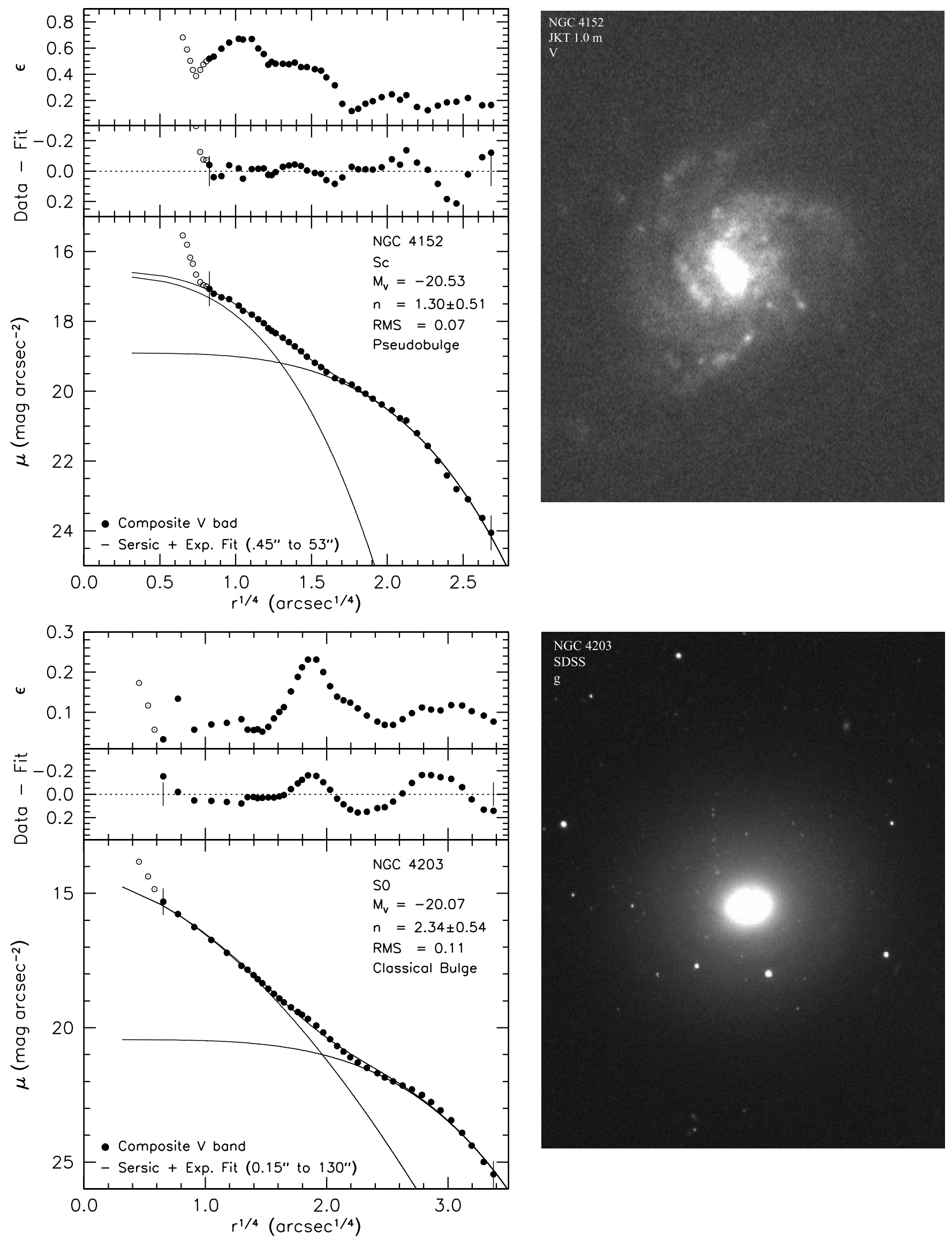

Figure 14. (Continued) 

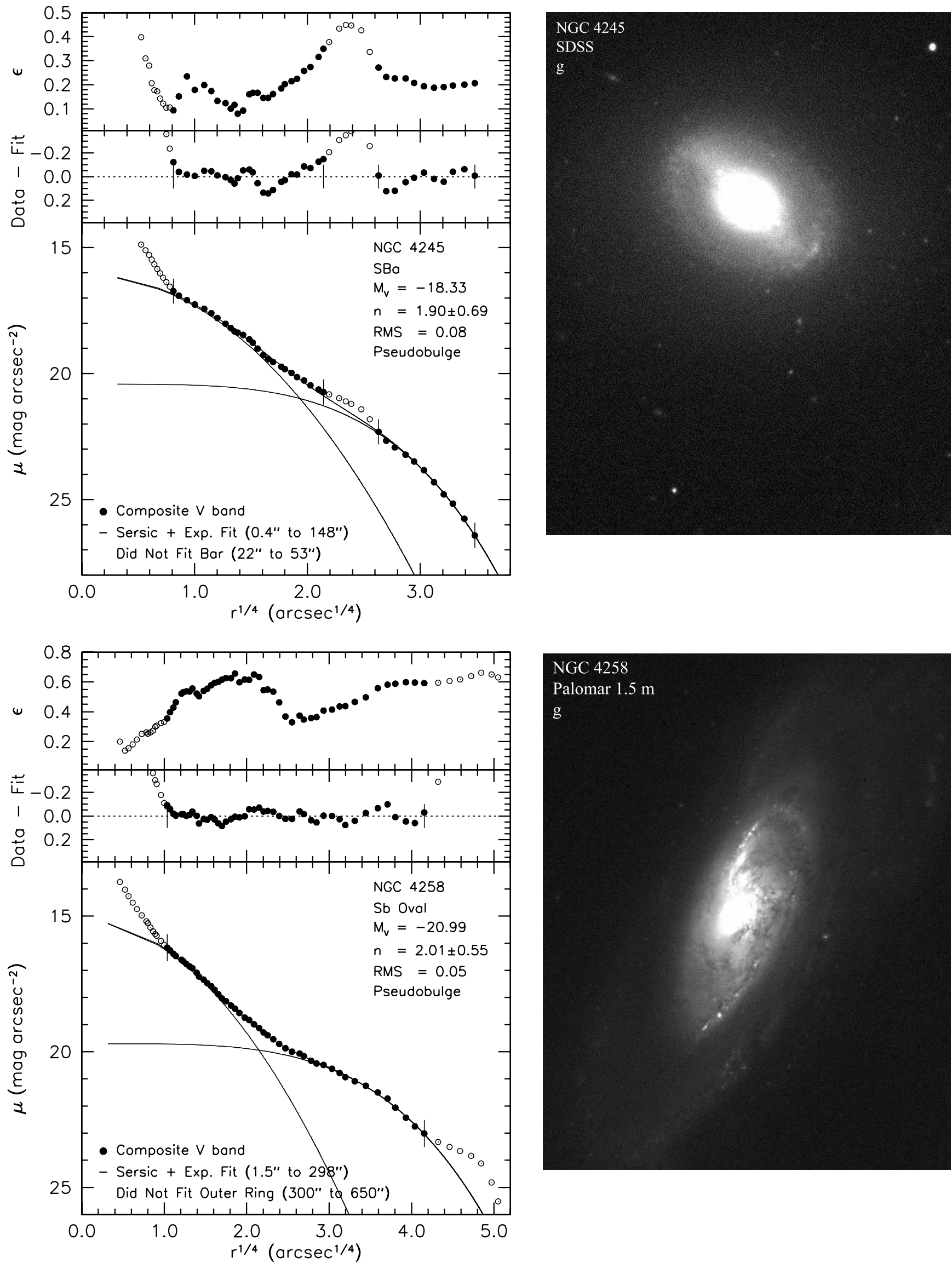

Figure 14. (Continued) 

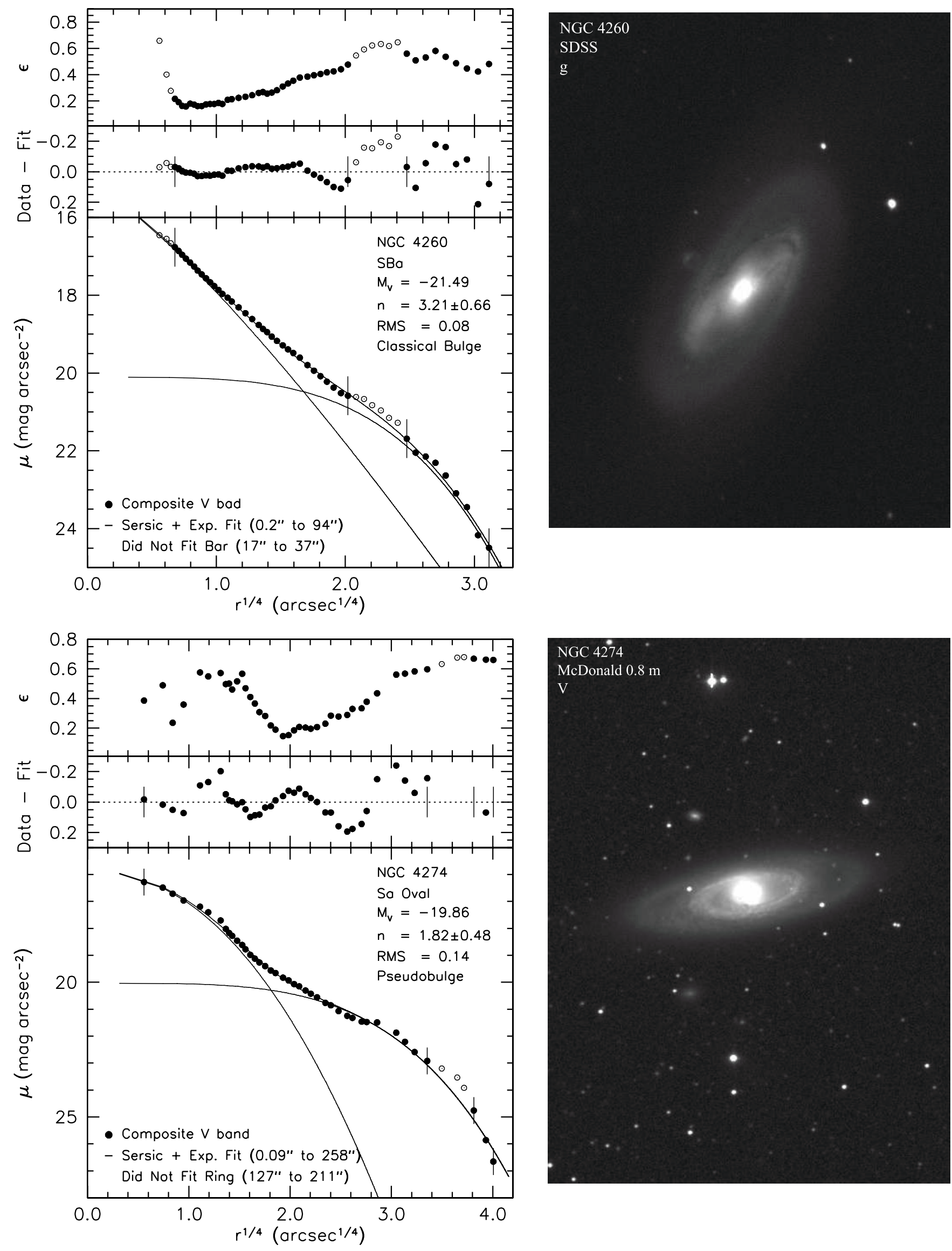

Figure 14. (Continued) 

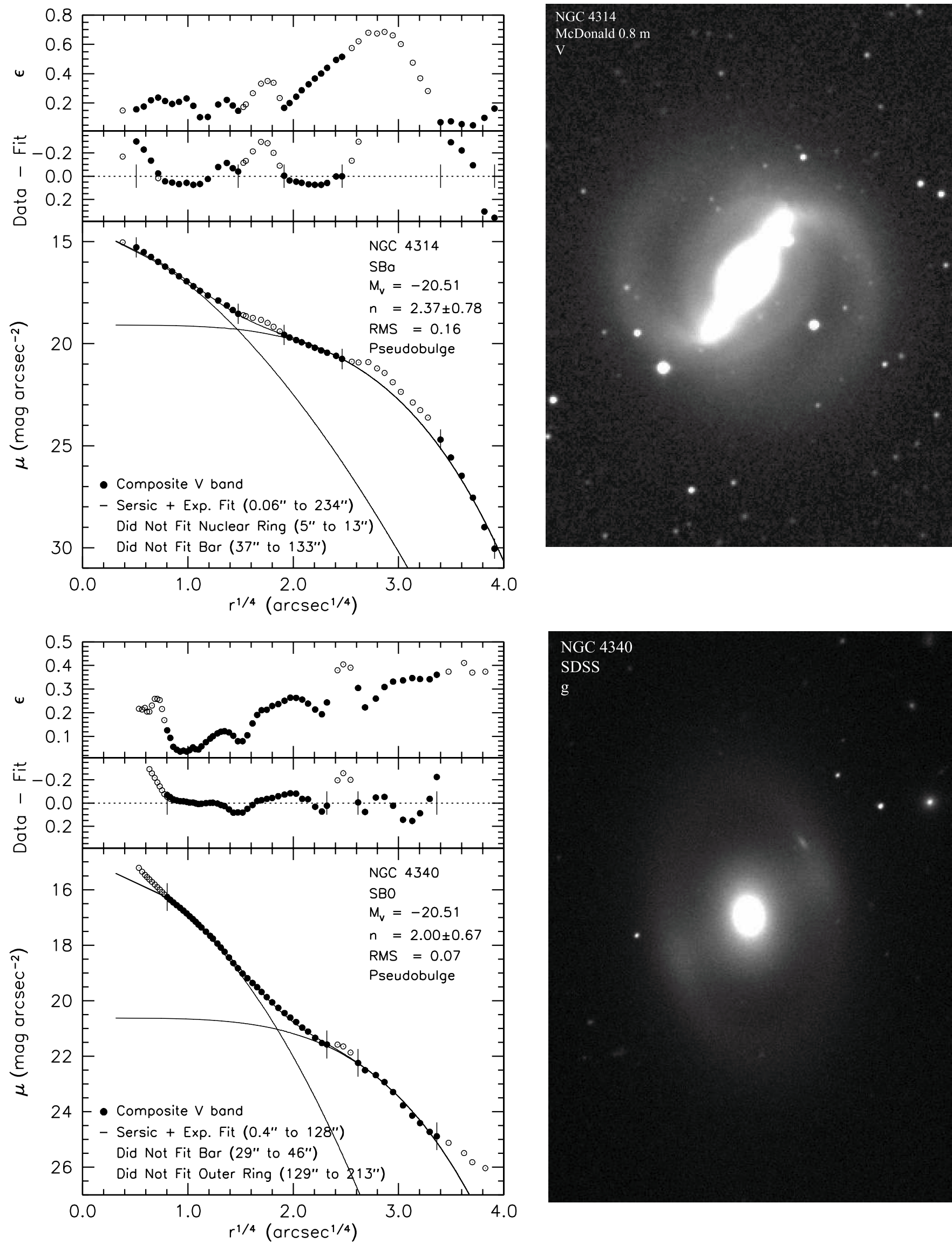

NGC 4340

SDSS

$\mathrm{g}$

Figure 14. (Continued) 

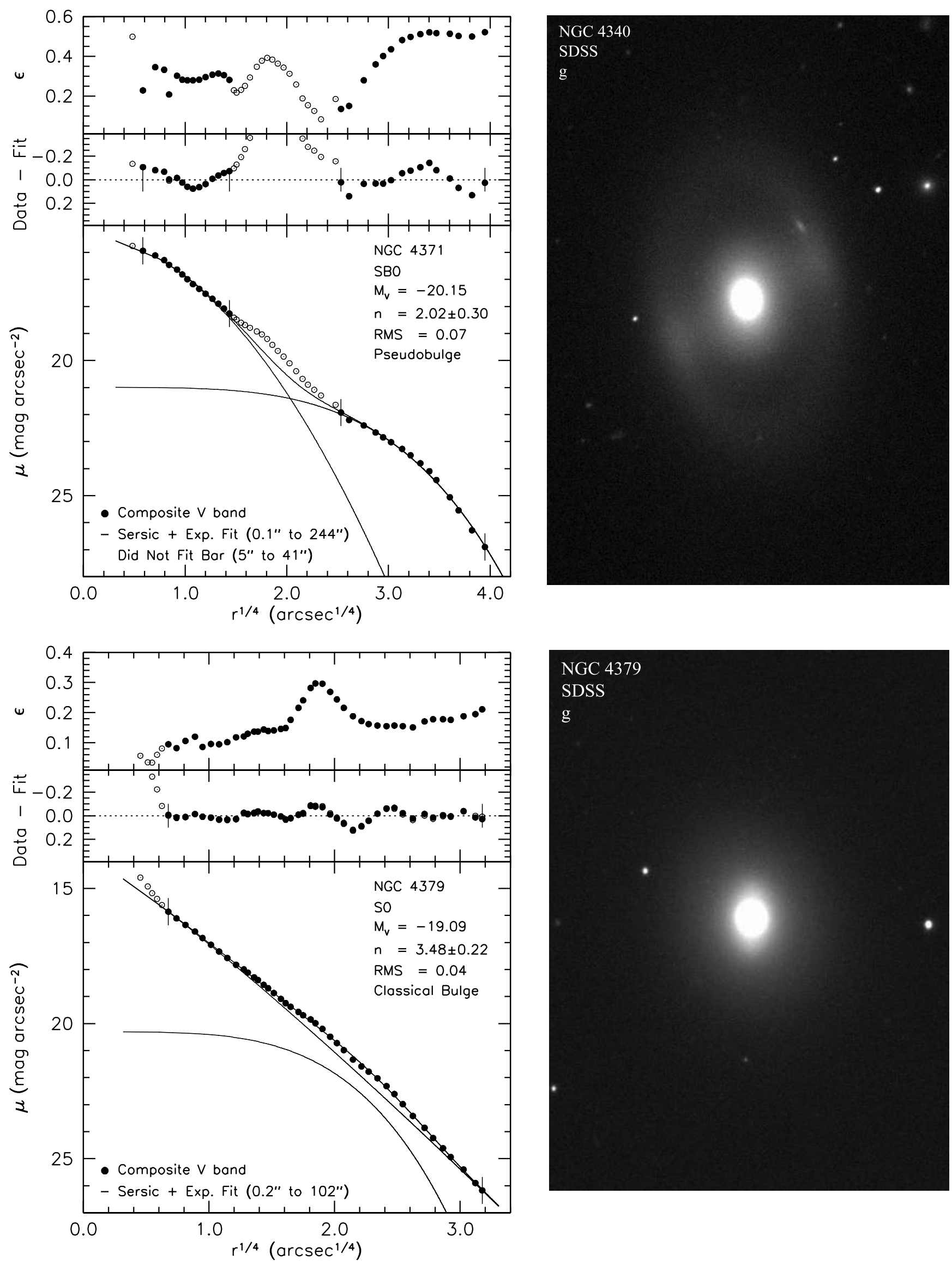

NGC 4379

SDSS

$\mathrm{g}$

Figure 14. (Continued) 

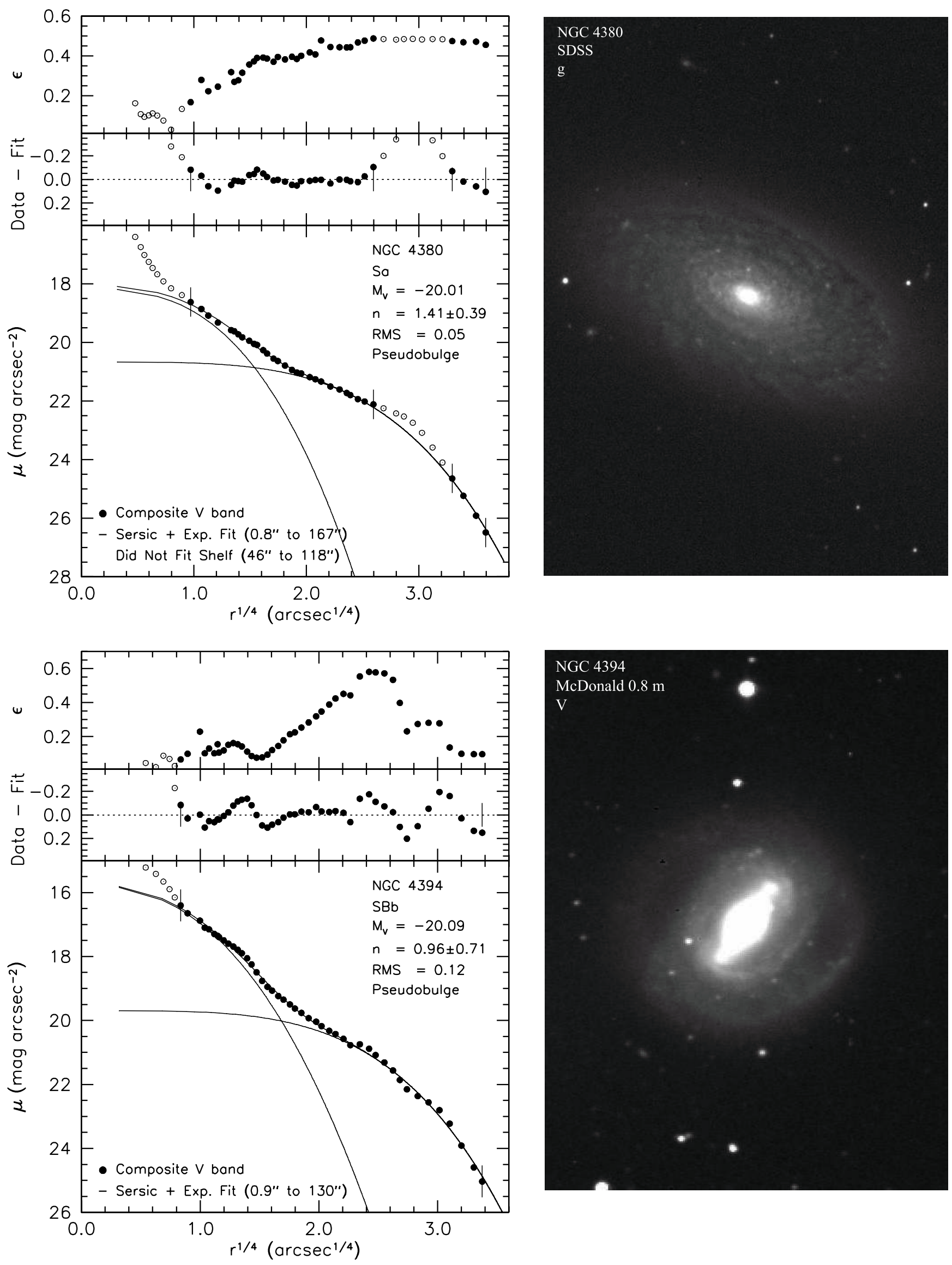

Figure 14. (Continued) 

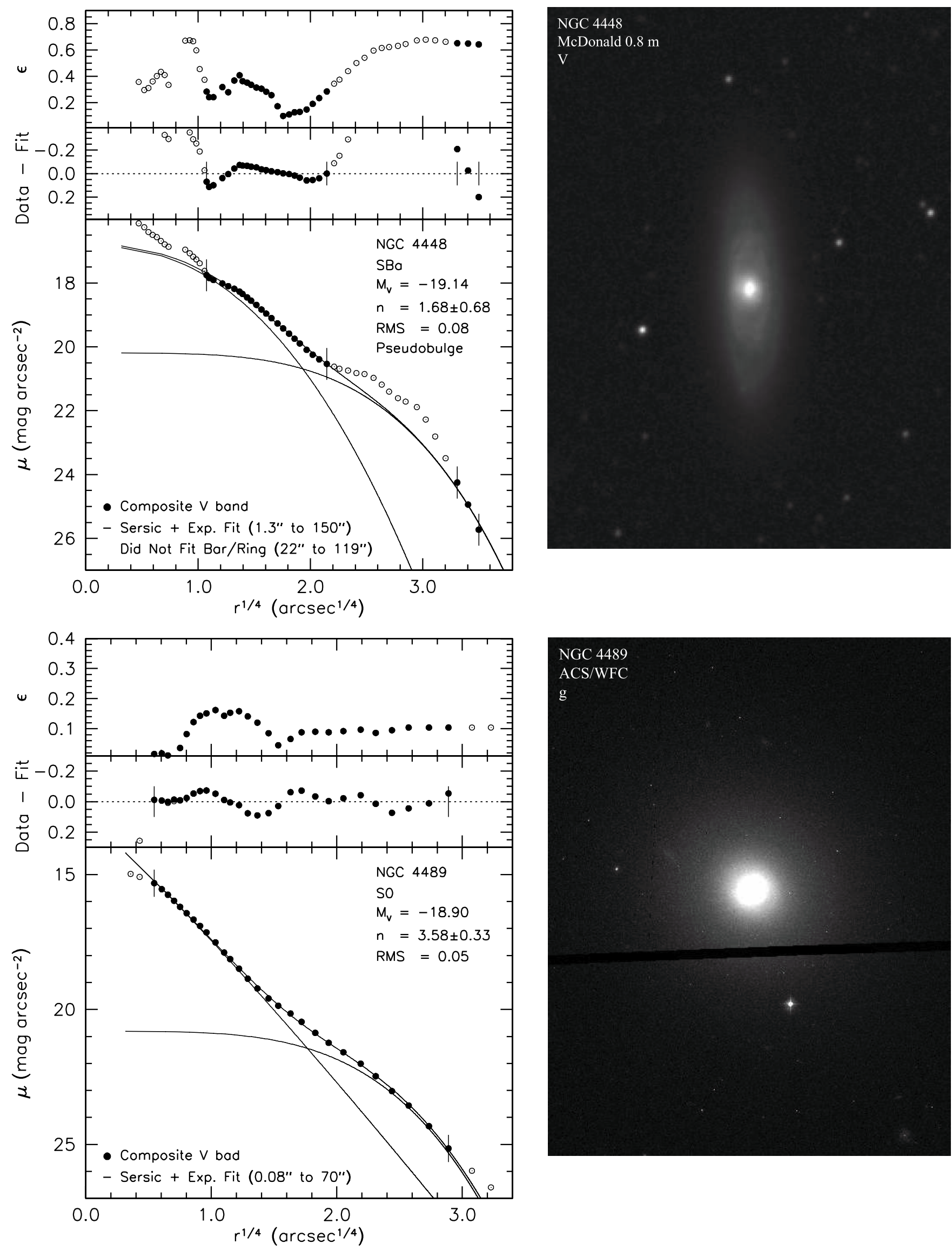

NGC 4489

ACS/WFC

$\mathrm{g}$

Figure 14. (Continued) 

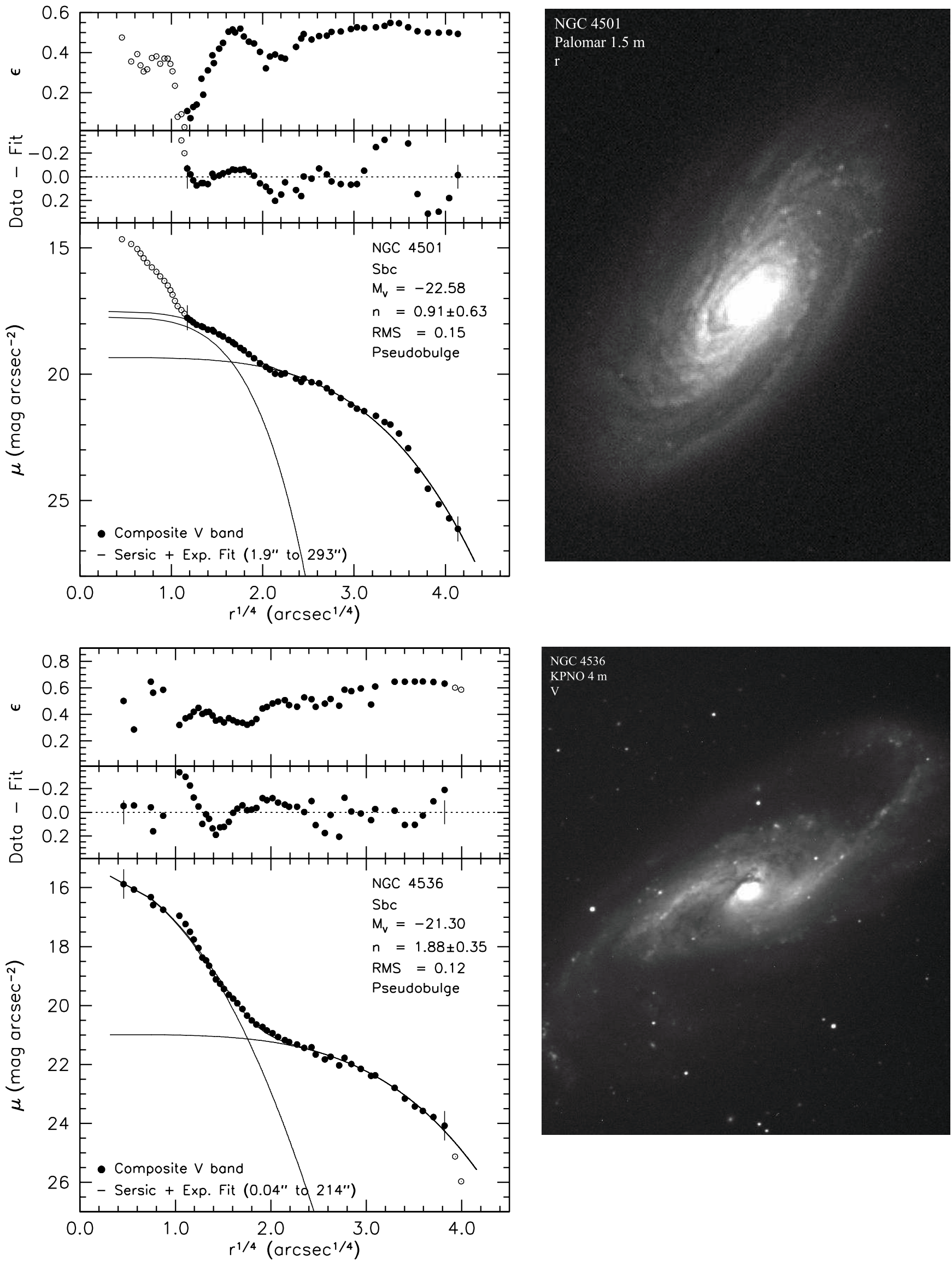

Figure 14. (Continued) 

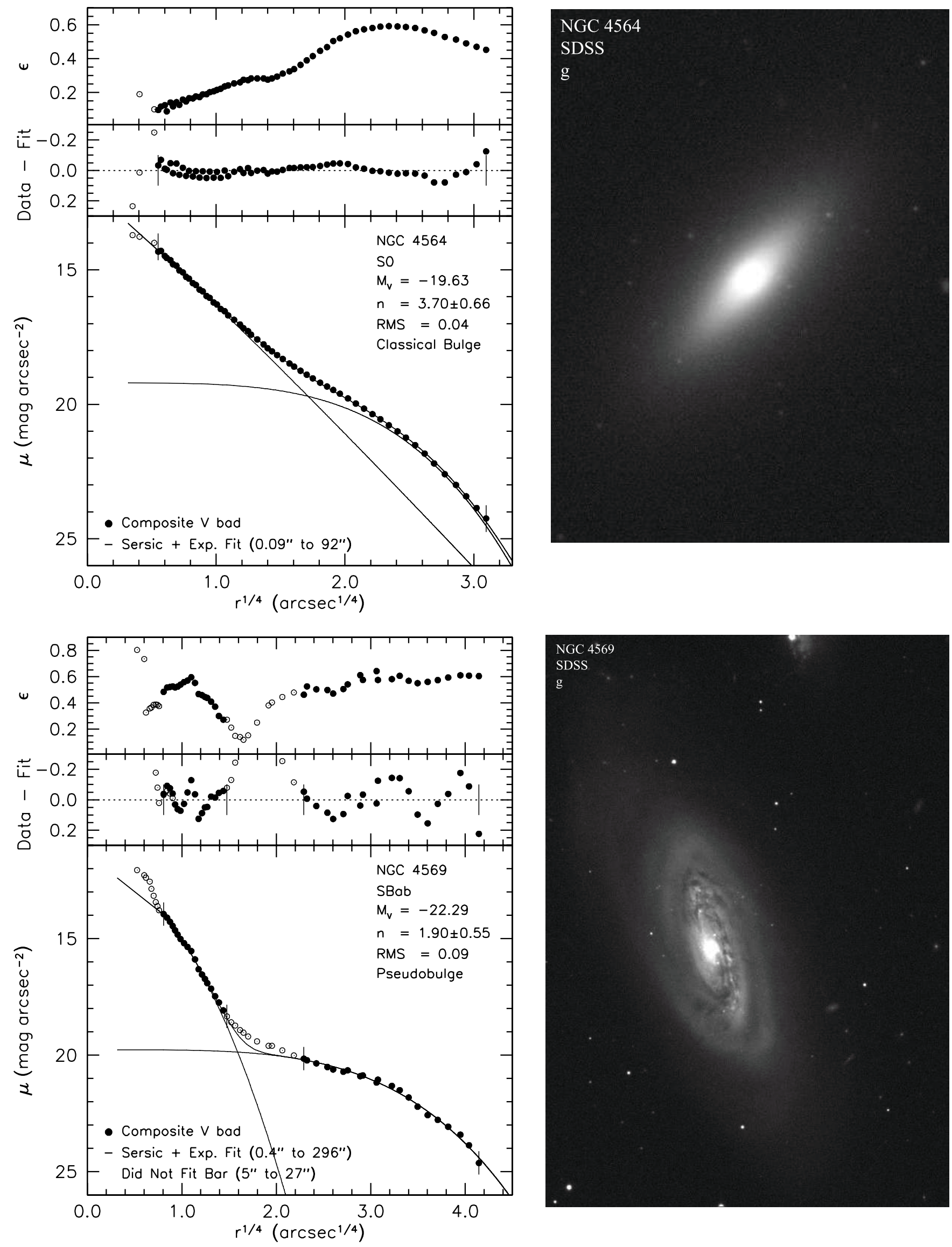

Figure 14. (Continued) 


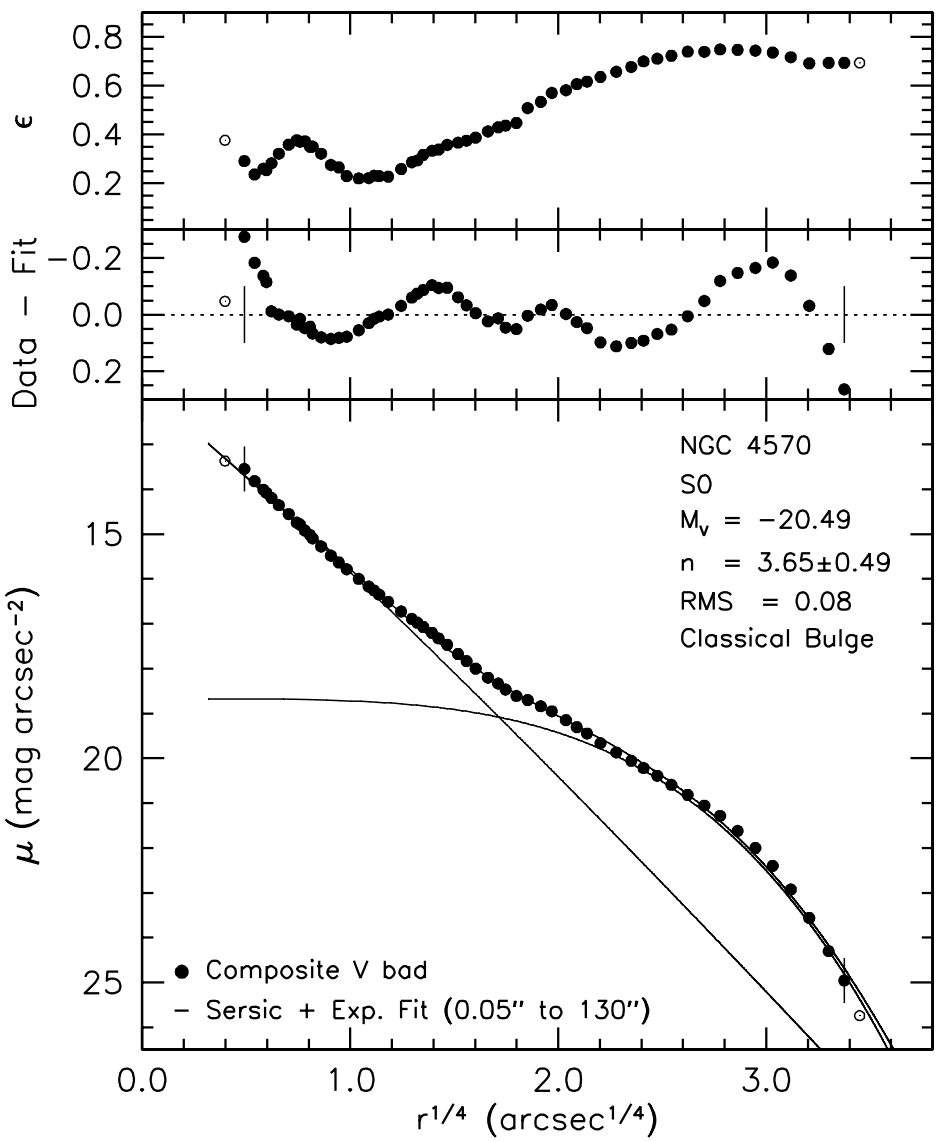

\section{NGC 4570 \\ SDSS \\ $\mathrm{g}$}
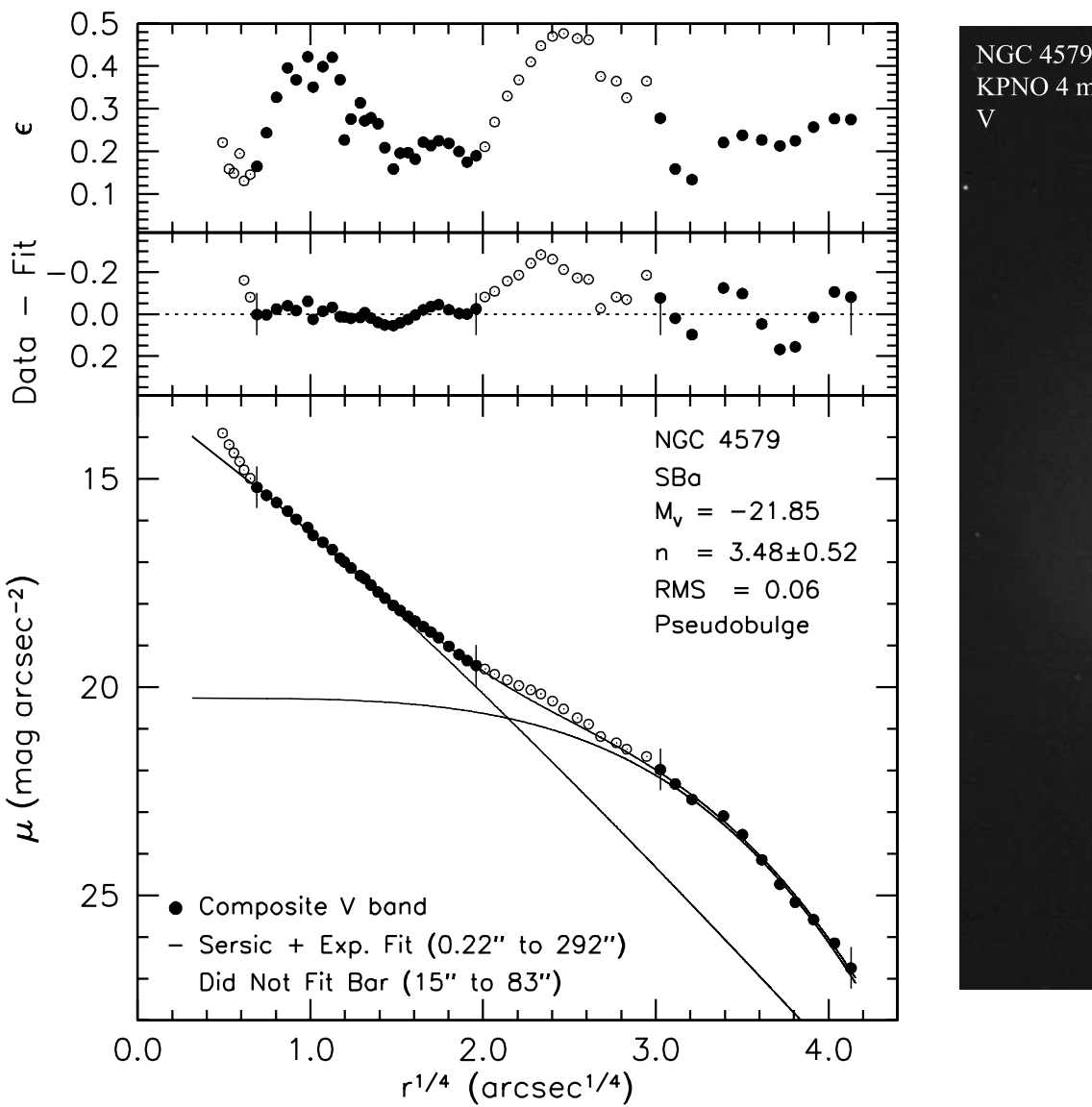

Figure 14. (Continued) 

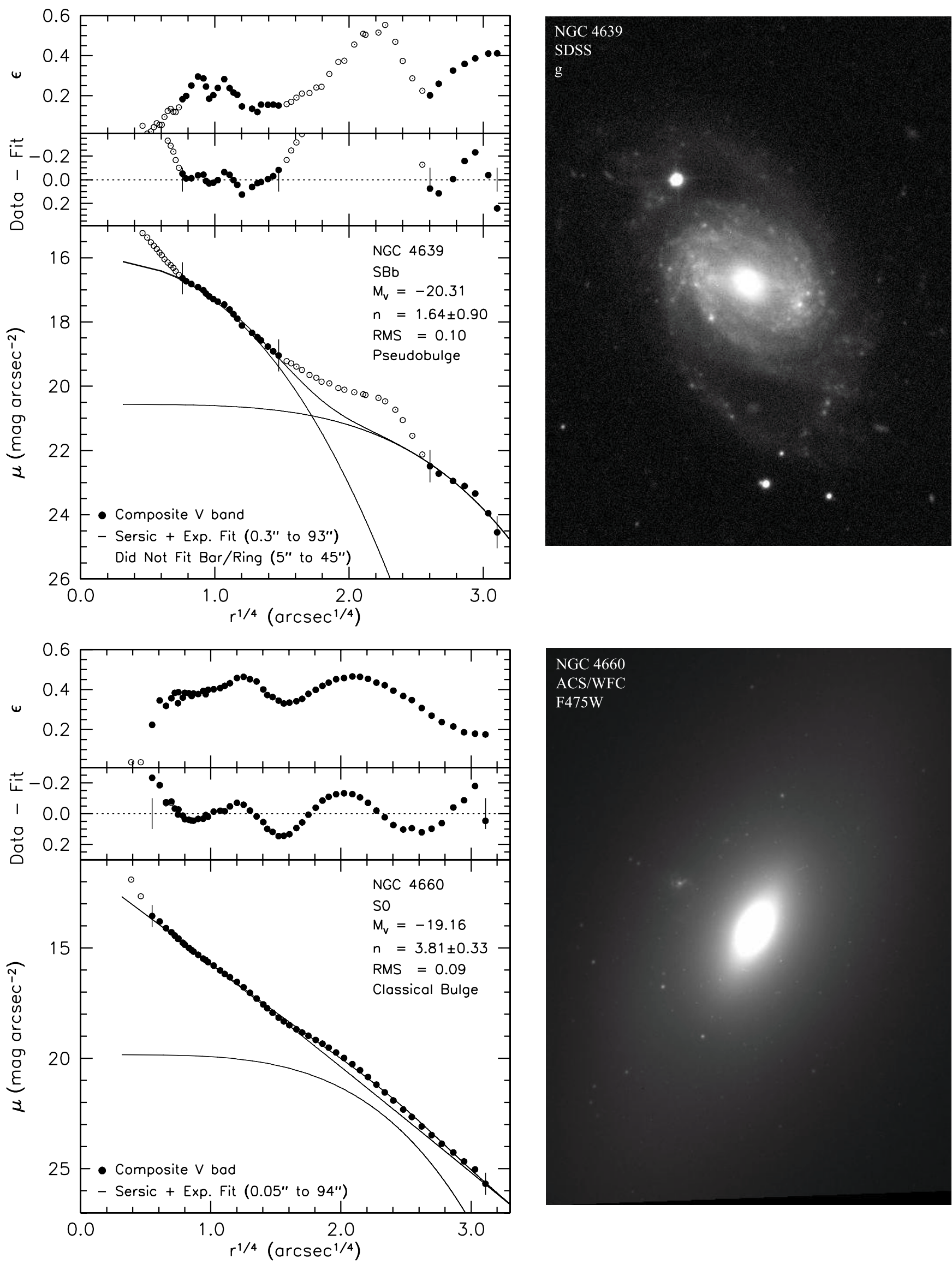

NGC 4660
ACS/WFC

F475W

Figure 14. (Continued) 

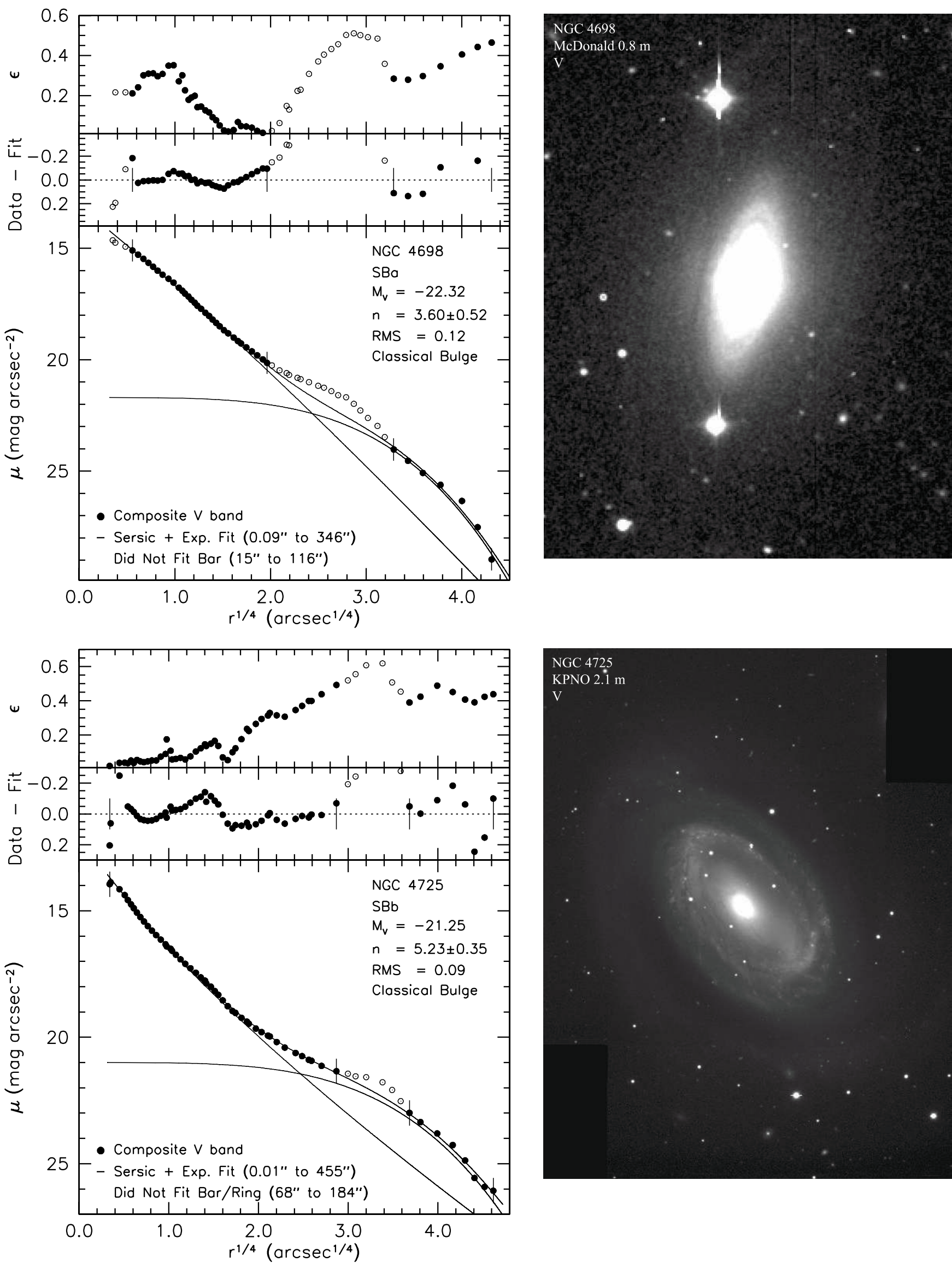

Figure 14. (Continued) 

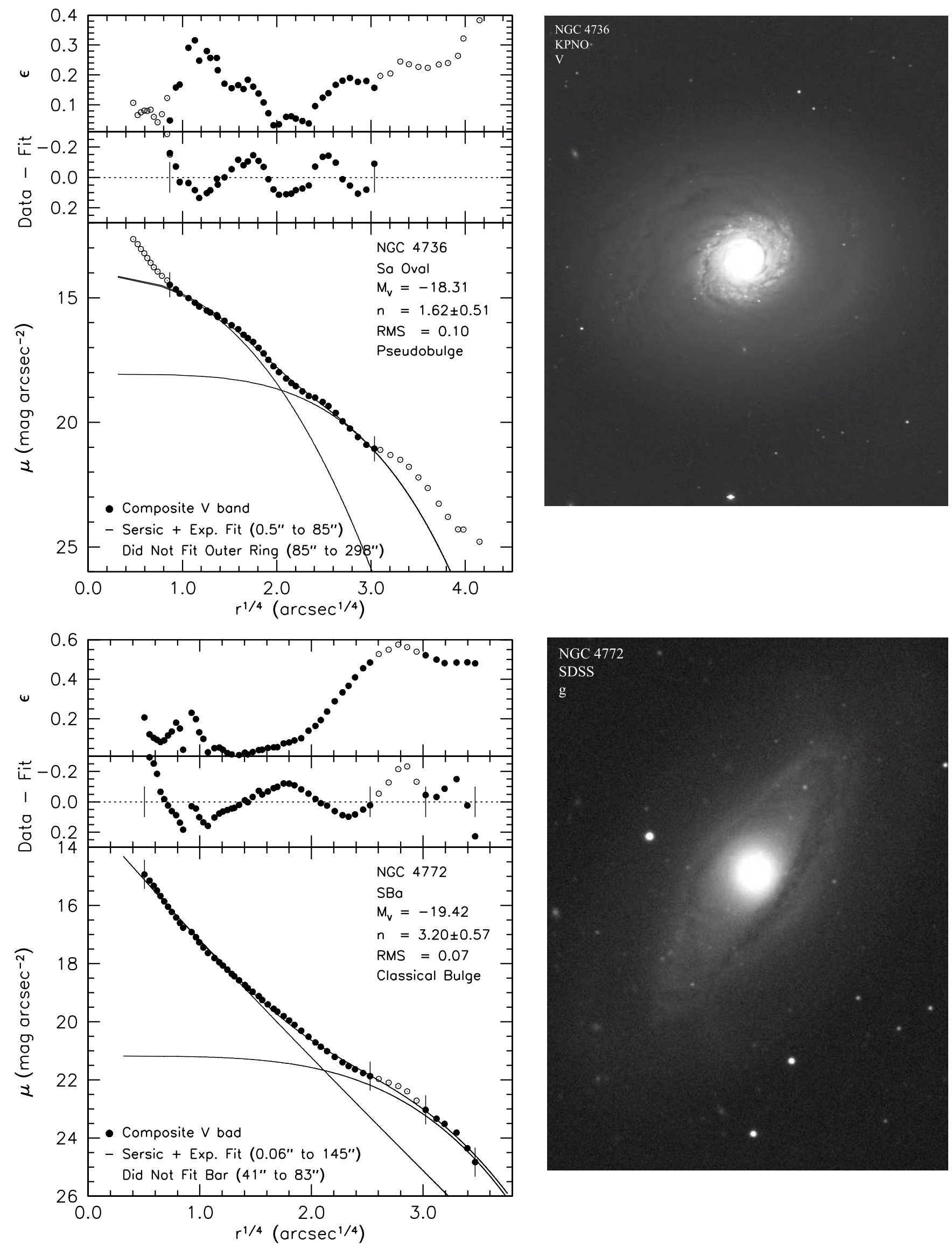

Figure 14. (Continued) 

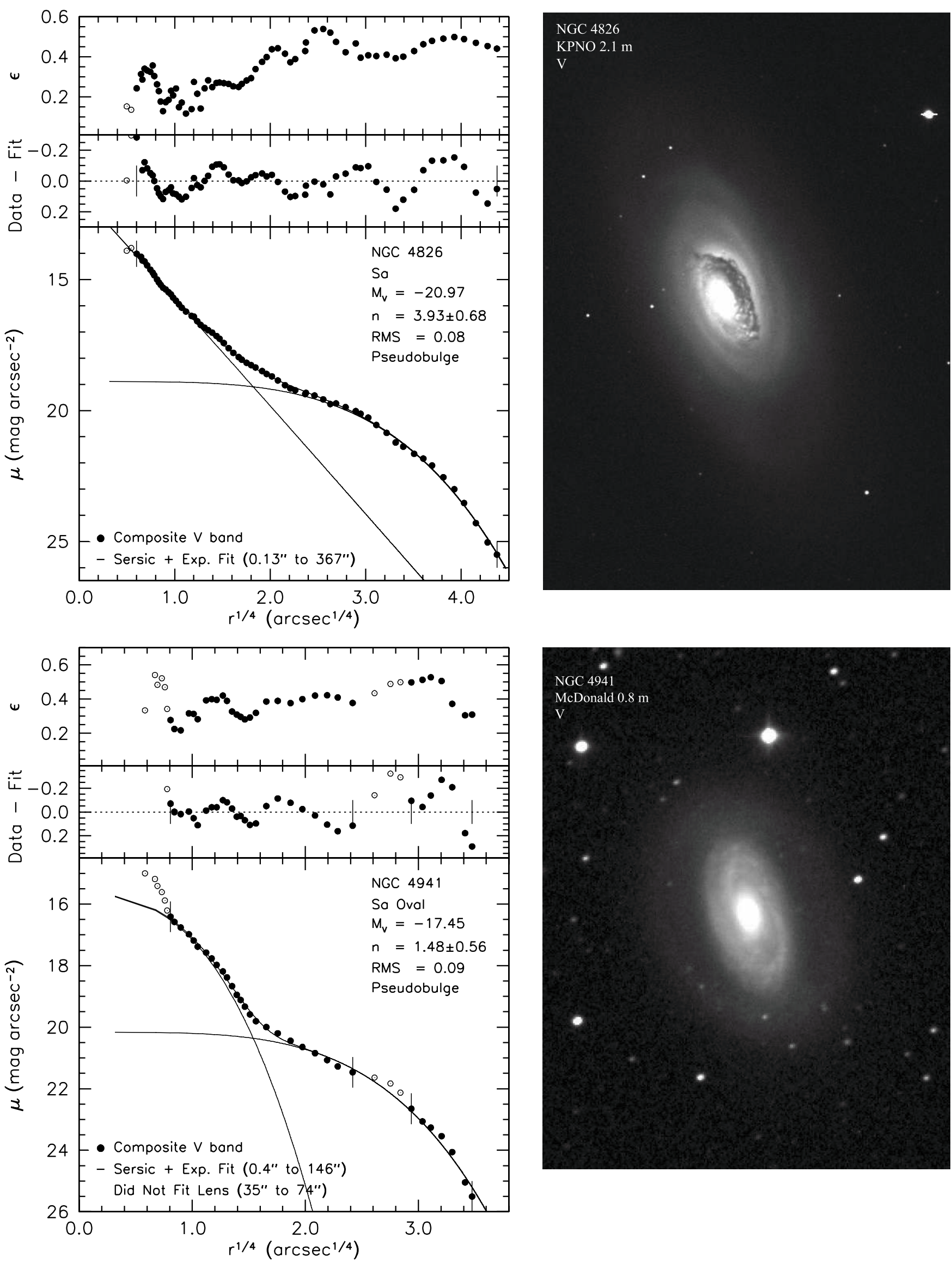

Figure 14. (Continued) 

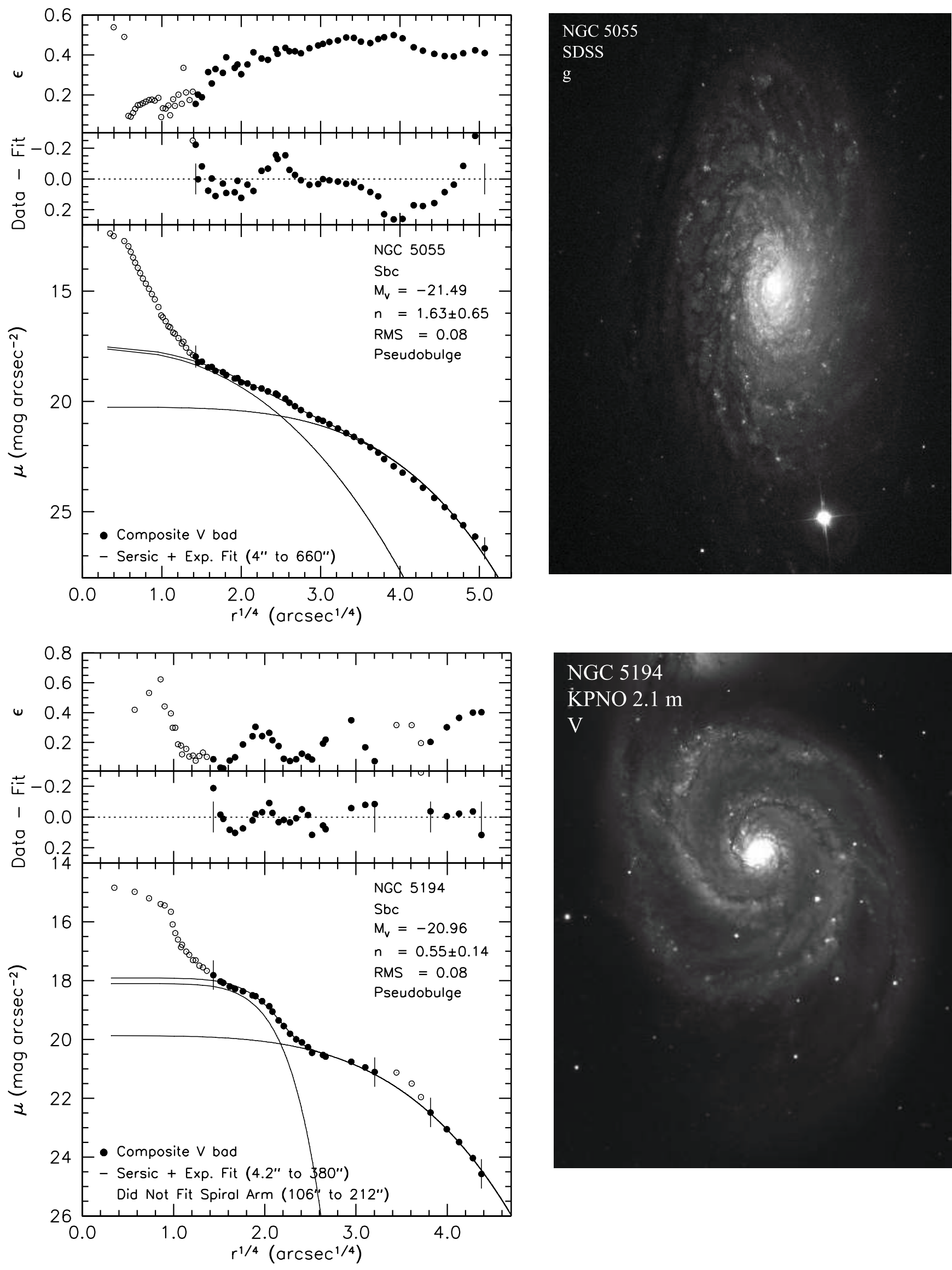

Figure 14. (Continued) 

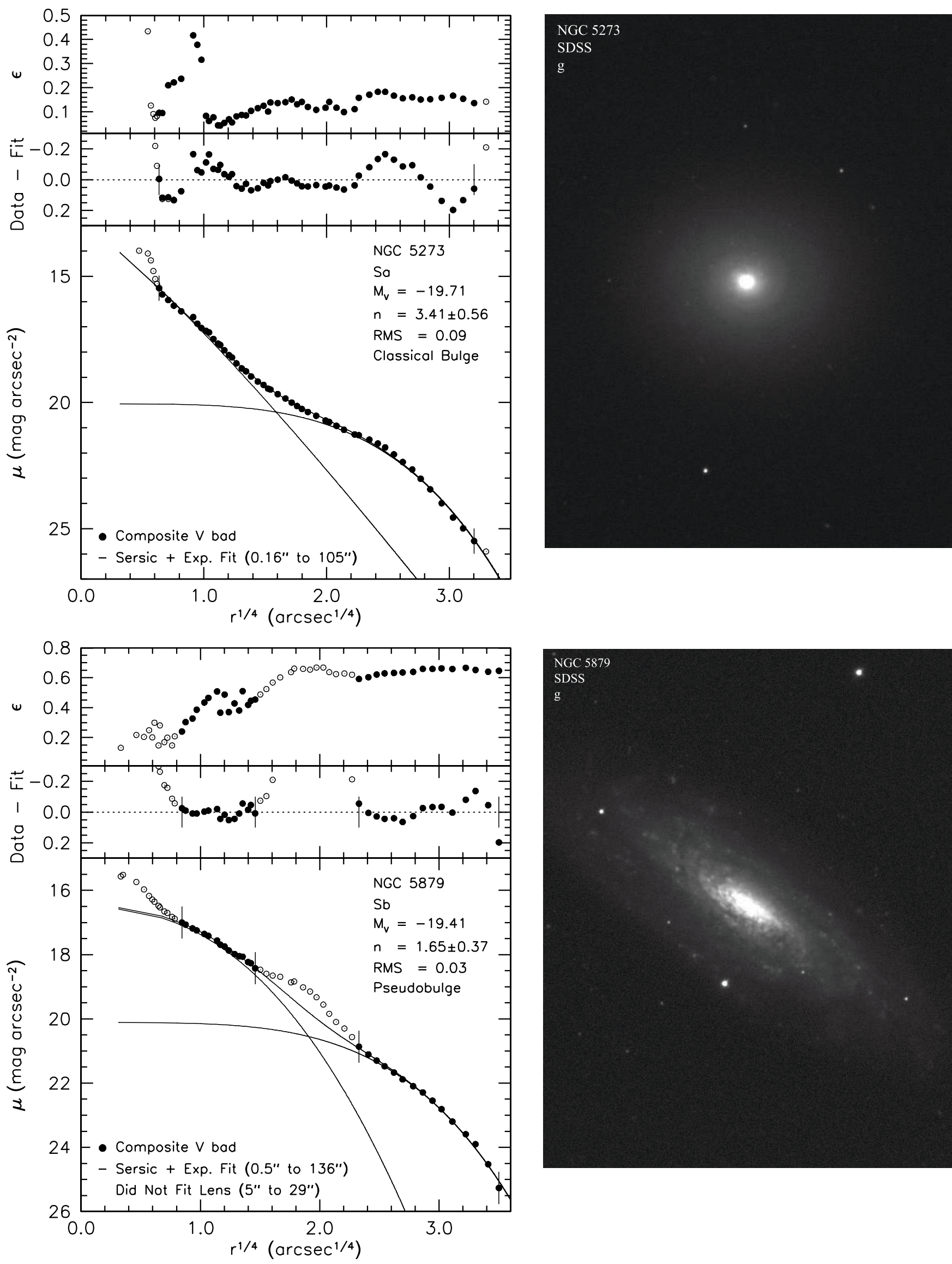

Figure 14. (Continued) 

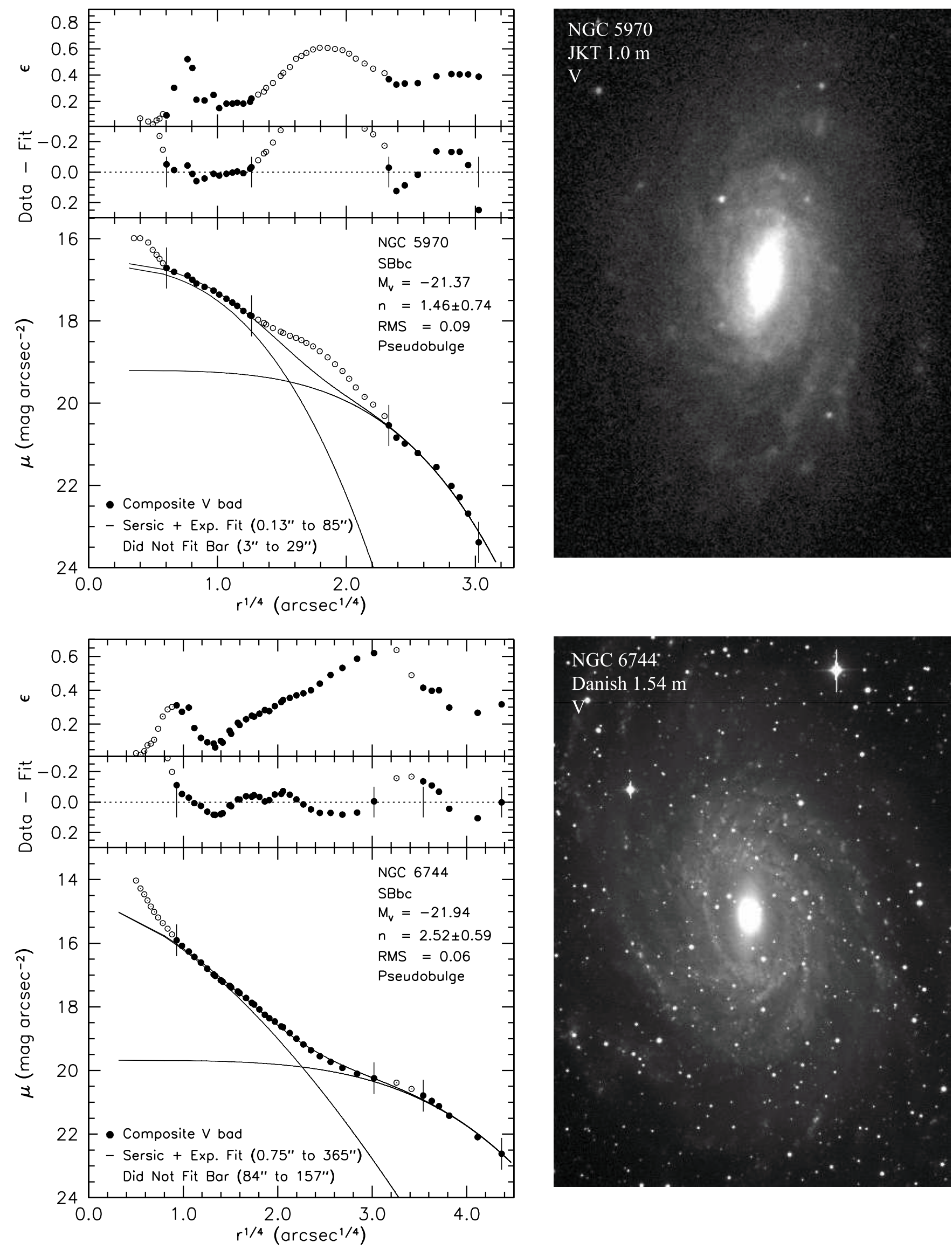

Figure 14. (Continued) 

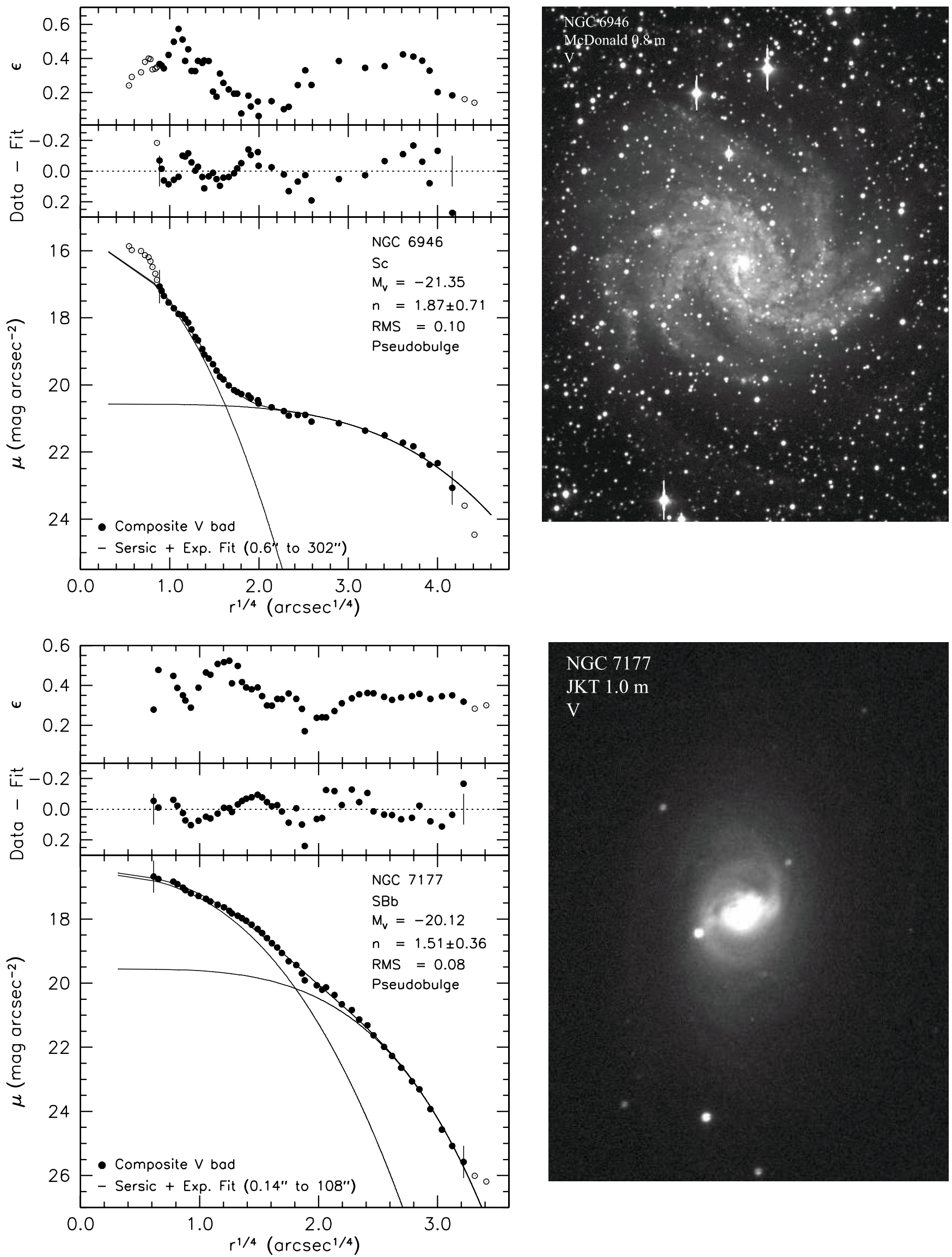

Figure 14. (Continued) 

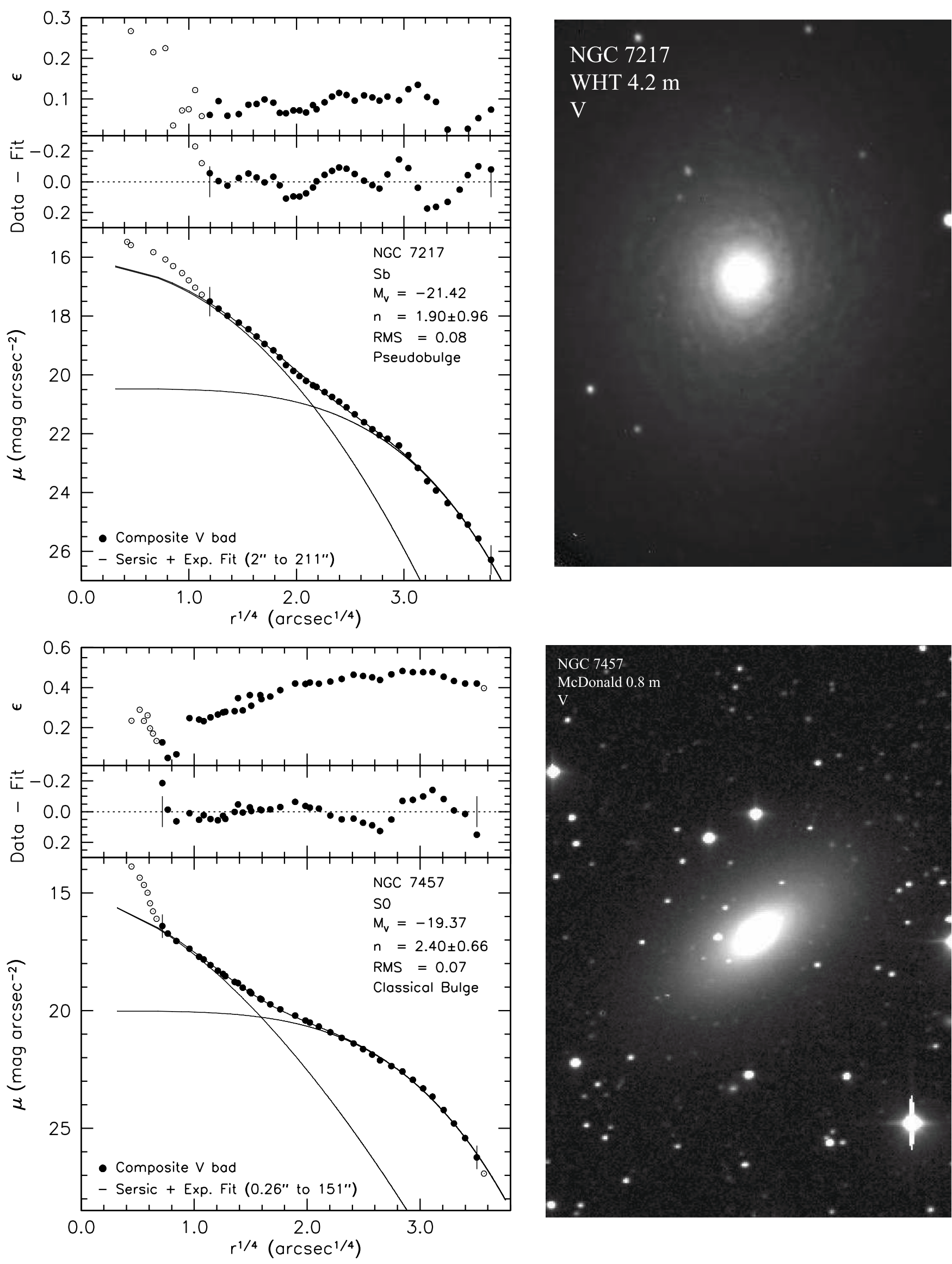

Figure 14. (Continued) 

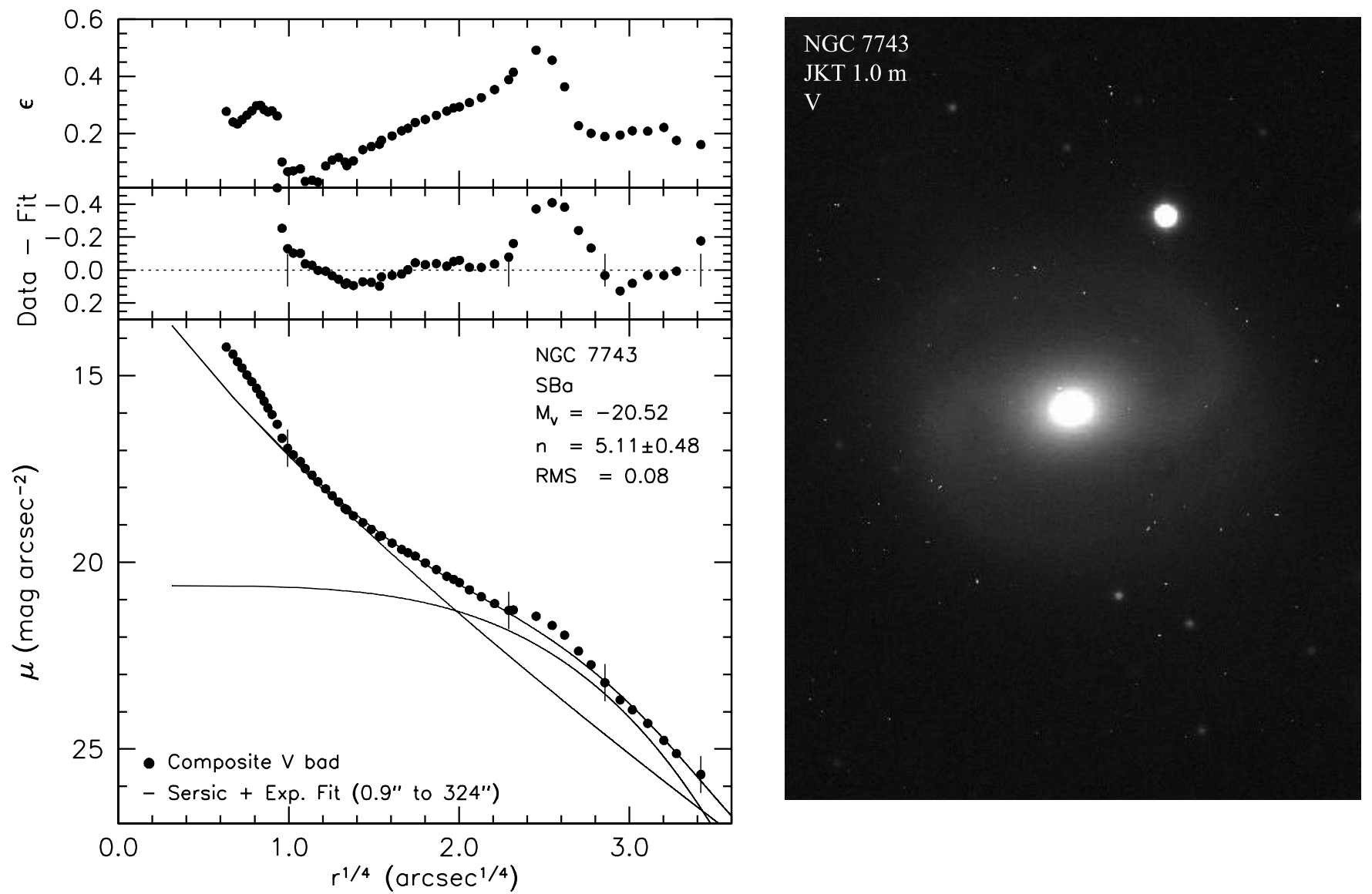

Figure 14. (Continued)

This research has made use of the NASA/IPAC Extragalactic Database (NED) which is operated by the Jet Propulsion Laboratory, California Institute of Technology, under contract with the National Aeronautics and Space Administration. This research has made use of the NASA/IPAC Infrared Science Archive, which is operated by the Jet Propulsion Laboratory, California Institute of Technology, under contract with the National Aeronautics and Space Administration. Some of the data presented in this paper were obtained from the Multi-Mission Archive at the Space Telescope Science Institute (MAST). STScI is operated by the Association of Universities for Research in Astronomy, Inc., under NASA contract NAS5-26555. Support for MAST for non-HST data is provided by the NASA Office of Space Science via grant NAG5-7584 and by other grants and contracts. Funding for the SDSS and SDSS-II has been provided by the Alfred P. Sloan Foundation, the Participating Institutions, the National Science Foundation, the U.S. Department of Energy, the National Aeronautics and Space Administration, the Japanese Monbukagakusho, the Max Planck Society, and the Higher Education Funding Council for England. The SDSS Web site is http://www.sdss.org/. The SDSS is managed by the Astrophysical Research Consortium for the Participating Institutions. The Participating Institutions are the American Museum of Natural History, Astrophysical Institute Potsdam, University of Basel, Cambridge University, Case Western Reserve University, University of Chicago, Drexel University, Fermilab, the Institute for Advanced Study, the Japan Participation Group, Johns Hopkins University, the Joint Institute for Nuclear Astrophysics, the Kavli Institute for Particle Astrophysics and Cosmology, the Korean Scientist Group, the Chinese Academy of Sciences
(LAMOST), Los Alamos National Laboratory, the Max-PlanckInstitute for Astronomy (MPIA), the Max-Planck-Institute for Astrophysics (MPA), New Mexico State University, Ohio State University, University of Pittsburgh, University of Portsmouth, Princeton University, the United States Naval Observatory, and the University of Washington. This publication makes use of data products from the Two Micron All Sky Survey, which is a joint project of the University of Massachusetts and the Infrared Processing and Analysis Center/California Institute of Technology, funded by the National Aeronautics and Space Administration and the National Science Foundation.

\section{APPENDIX A}

\section{PROFILES AND IMAGES OF SAMPLE GALAXIES}

Here we show all bulge-disk decompositions reported in this paper (Figure 14). All profiles are plotted against $r^{1 / 4}$ in arcseconds. As stated earlier we do not fit nuclei, bars, rings, lenses, and other distortions to surface brightness profile. Those data points included in the fitting are represented by filled circles, those not included in the fitting are represented by open circles. We also show representations of the model fit to the galaxies, as black lines. We show the Sérsic function bulge, the exponential disk, and the combination (Equation (1)).

Each figure gives the name of the galaxy, the Hubble type, the total absolute $V$-band magnitude, the Sérsic index of the bulge, the rms deviation of the fit (in mag $\operatorname{arcsec}^{-2}$ ), and the classification of the bulge. We also note (in the lower left) the region of the fit, and any data not included are noted as well. 


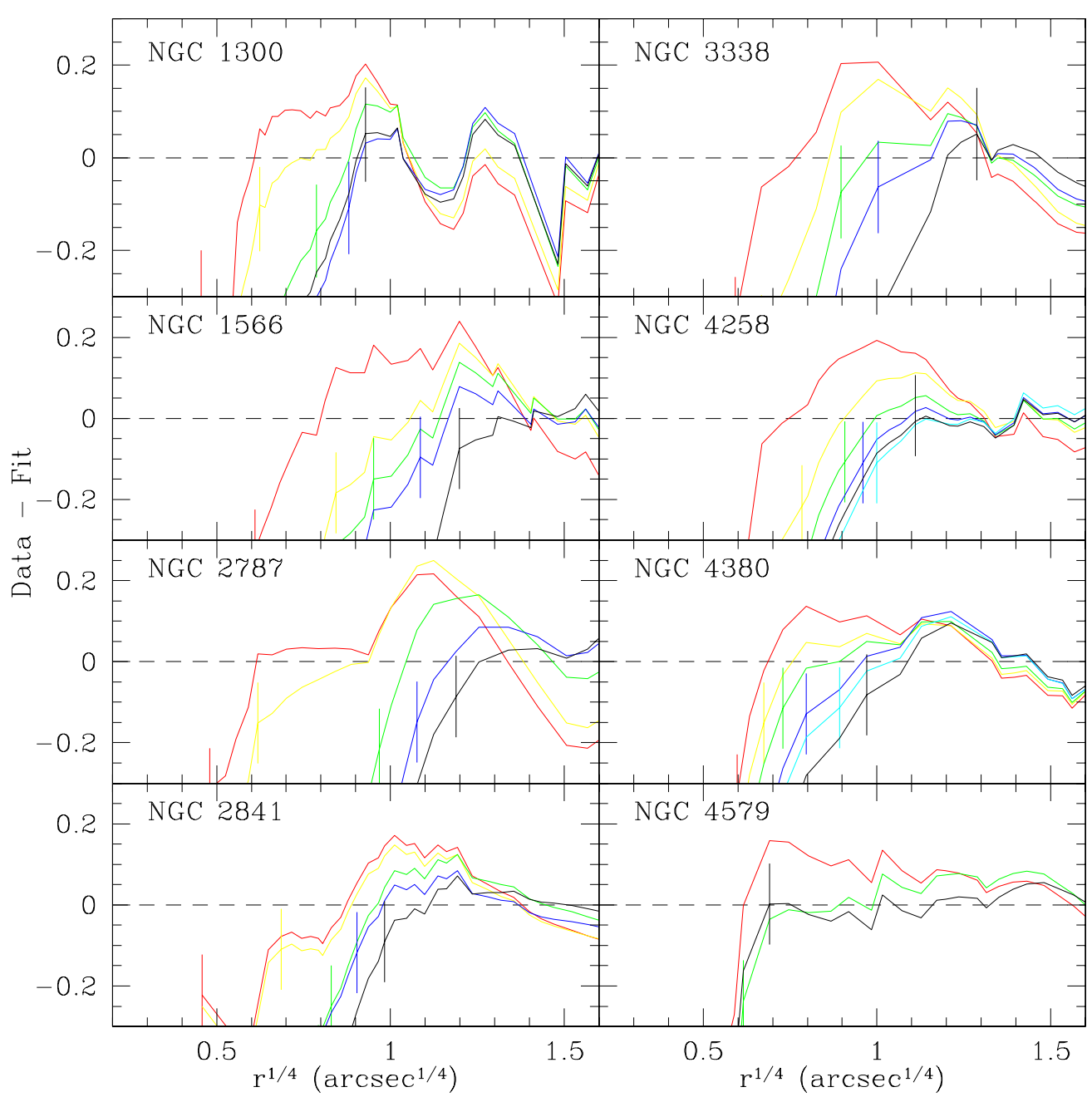

Figure 15. Profiles of the residuals of data minus decompositions for the innermost $\sim 10$ arcsec of eight galaxies. In each panel the red lines indicate the residuals of the initial fit without accounting for the nucleus, and the black lines indicate the residuals of the final fit. Other colors indicate intermediate iterations (yellow, green, blue, and cyan). The radius at which the fit begins is marked on each profile by a vertical mark $\left( \pm 0.1 \mathrm{mag} \operatorname{arcsec}^{-2}\right)$.

For each galaxy we also show an image of the galaxy. Each image indicates the telescope from which it came and the filter used for the image. The images are shown such that readers can associate those structures not fit with structures in the images (such as bar).

\section{APPENDIX B}

\section{CHOICE OF FITTING RANGE: NUCLEAR DEVIATIONS}

Nuclear star cluster (nuclei) are commonly found in intermediate- and late-type galaxies (Carollo et al. 2002; Böker et al. 2002). They are often easily distinguished from the bulge; they have much smaller effective radii and have higher effective surface brightness. When carrying out bulge-disk decompositions nuclei must be accounted for in some way. If included in the fit, a nucleus will increase the bulge Sérsic index, $n_{b}$.

Nuclei are quite compact; their surface brightness profiles fall off rapidly with radius. Thus, one can exclude the region of the surface brightness profile from the fit to Equation (1), without losing much of the dynamic range of the fit. An alternative method is to include an extra component in the fitting routine (e.g., Balcells et al. 2003). We feel both are good methods, and both have advantages and disadvantages. For simplicity, we choose to remove the nucleus from the fit.
In Figure 15, we show how we choose the radius over which the nuclear component is excluded from the fit. First, a decomposition is attempted using the entire profile. A significant deviation $\left(\sim 0.3 \mathrm{mag} \operatorname{arcsec}^{-2}\right)$ from the fit in the center of the galaxy is interpreted as indicative of a nucleus. The desire is to find the radius at which the galaxy begins to be well described by an inner Sérsic bulge plus outer exponential disk; thus we move the fitting radius to the first point at which the deviation is within a $0.1 \mathrm{mag} \mathrm{arcsec}{ }^{-2}$, which is the typical rms deviation of galaxies in our fit.

In Figure 15, we show a range of examples of this process. In each panel the red lines indicate the residuals of the initial fit without accounting for the nucleus, and the black lines indicate the residuals of the final fit. Other colors indicate intermediate iterations (yellow, green, blue, and cyan). NGC 4579 shows a very small nucleus that converges to a small residual quite quickly. In contrast, NGC 1566 shows a very large nucleus, and without an objective method, the choice of the fitting region may be somewhat arbitrary. In each of these a key characteristic is that subsequent fits not only show smaller deviation in the center, but also have smaller residuals over the entire profile. NGC 2841 shows an example in which the overall residual drives us to choose to exclude a larger radius from the fit. The second fit (the yellow line) shows large residuals with apparent 

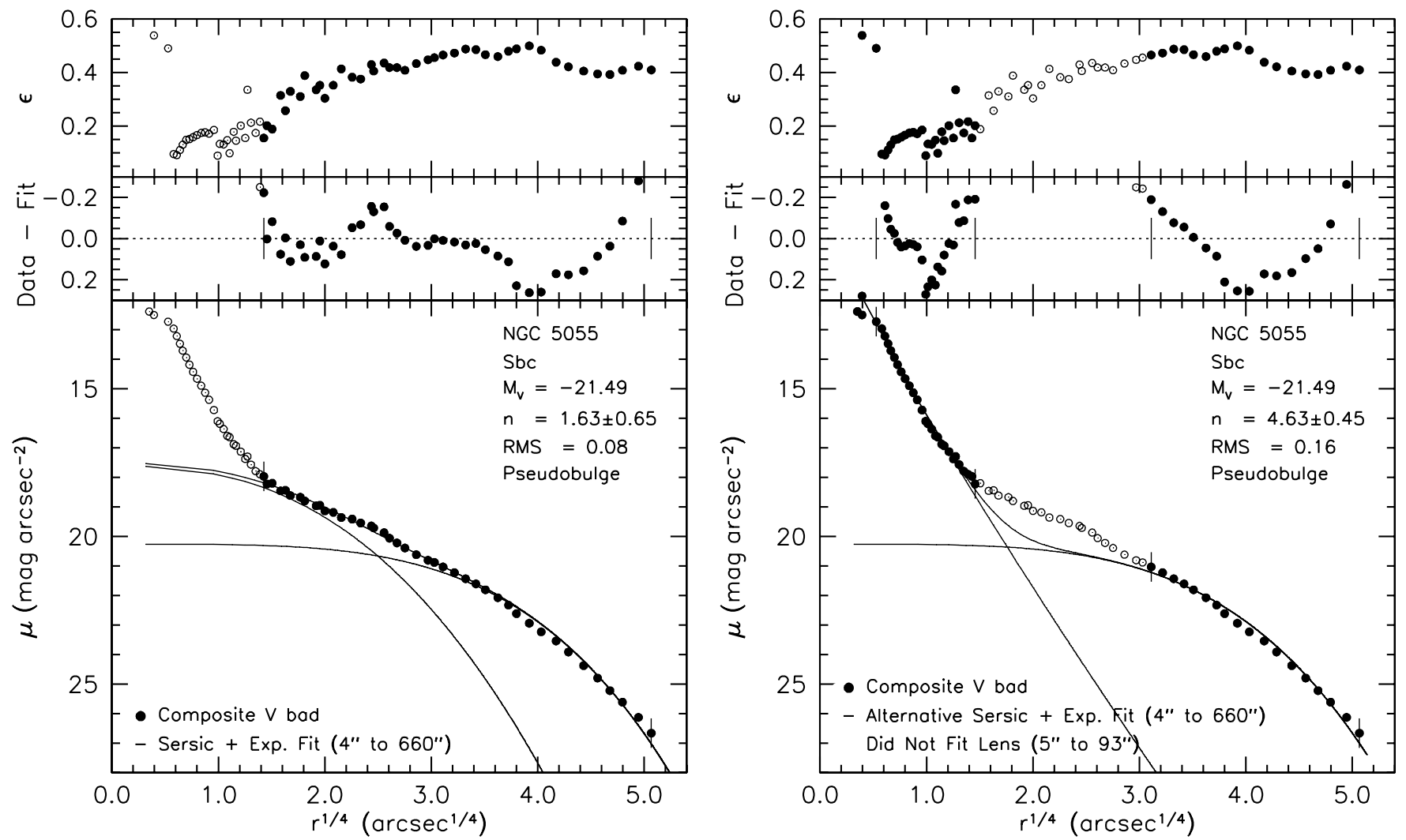

Figure 16. Two separate interpretations of NGC 5055 (see the text). On the left, the center is interpreted as a nucleus. On the right, the center is interpreted as a bulge, and a shelf in the surface brightness profile is excluded.

substructure. This is interpreted as indicating the presence of a nucleus, which is removed from the fit.

NGC 5055 is a special case, as illustrated in Figure 16. This galaxy has an unusually bright nucleus for an intermediatetype galaxy (the left panel of Figure 16). Also, the nuclear component extends over a larger fraction of the galaxy's radius than is typical. The alternative interpretation is that this nucleus is the bulge, and it is surrounded by a lens that is apparent in the surface brightness profile (the right panel of Figure 16). The first interpretation results in a lower rms deviation, and we choose this one. We do note that in this case the choice of a nucleus is coincident with our result in this paper, and that pseudobulges typically have smaller Sérsic index. It is entirely possible that this galaxy is another example of a system with both a classical and pseudobulge. A more detailed study may be necessary to understand this galaxy.

\section{APPENDIX C}

\section{CHOICE OF FITTING RANGE: BARS, RINGS, AND LENSES}

We now consider the effects of not including non-nuclear deviations to the surface brightness profiles such as bars and rings in galactic disks. This practice is largely subjective. Also, this practice assumes that the shapes of underlying bulges and disks in barred galaxies are not fundamentally different than those without bars, effectively treating the bars as additional components. Alternative methods, such as parameterization of the bar, and 2D fitting are possible, but each has its shortfalls.

Here, we investigate the effects and biases of our choice to remove significant deviations to the initial best-fit bulge-disk decomposition (Equation (1)) for each galaxy. These deviations are most commonly bars; however many other phenomena including rings, bright spiral structure, and lenses lead to similar deviations from the smooth fit. Also, it is sometimes the case that a bar is easily evident in an image, yet it is not identifiable in the profile. However, we can typically identify bars with increase in the ellipticity profile.

In Figure 17, we show the distribution of the sizes of those regions removed from the fits (i.e., holes). The interested reader can investigate them in Figure 14; they are denoted by vertical hatch marks and open circles. We find that holes come in two varieties: those which only affect the surface brightness of the disk and those which affect the surface brightness of the location in the profile where the bulge and disk are at equal surface brightness. In the figure, $\Delta \log ($ hole $)=\log \left(r_{\text {out,hole }}\right)-$ $\log \left(r_{\text {in,hole }}\right)$, where $r_{\text {in,hole }}$ is where the distortion to the surface brightness profile begins and $r_{\text {out,hole }}$ is where the distortion ends. Similarly, $\Delta \log$ (fit) refers to the range of radius included in the fit. Fitting is done in logarithmic radius, and thus is the more appropriate radial coordinate, in this instance. The largest exclusion is $45 \%$ of the fit radius. The distribution of all exclusion sizes is shown as the black outline, the distribution of bars in the outer disk is shown in cyan, and the distribution of those occurring near the radius at which the bulge and disk are equal are shown as green shading. It is interesting to note that those bars occurring near the bulge affect the dynamic range of the fit more than those that only affect the outer disk.

We attempt to be as conservative as possible when choosing what radii to exclude. Nonetheless, it is possible that we exclude points unnecessarily. To study the effect of this, we take four galaxies that do not have significant distortions (NGC 1325, NGC 1425, NGC 2841, and NGC 4203) and remove points to 


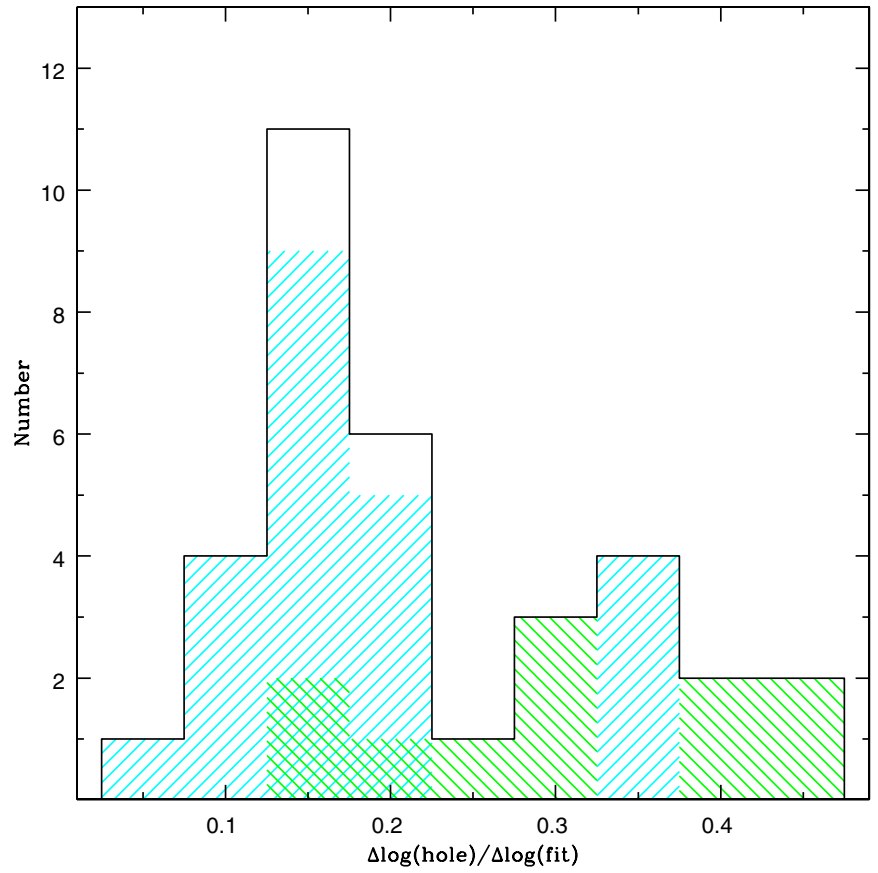

Figure 17. Distribution of the sizes of excluded regions from fits. The distribution of all exclusion sizes is shown as the black outline, the distribution of bars in the outer disk is shown in cyan, and the distribution of those occurring near the radius at which the bulge and disk are equal is shown as green shading. artificially create the effect of a bar in the surface brightness profile. We iteratively fit the profile and follow the effect on the fit parameters as we increase the size of the excluded region. We are principally interested in the effect on the Sérsic index which is shown in Figure 18. The break radius, $r_{0}$ in Equation (1), is tightly coupled with $n_{b}$ and thus can absorb changes to the profile, so we show $r_{0}$ as well. The result is relatively encouraging. The larger effect occurs in those fits which mimic distortions to the outer disk only. In these the general trend is to increase the Sérsic index. This reinforces the necessity to complement high-resolution data with the widest, deepest photometry one can obtain. We note that Figure 17 shows that only a few disk-only distortions are larger than $30 \%$ of logarithmic radial range of the fit, but this is enough to artificially increase $n_{b}$ as much as $n_{b} \sim 1$. The effect of removing points near the bulge-disk transition radius appears to affect the fit to the profile relatively little. Taking this into consideration, it seems that, if anything, removing too many points is likely to drive the Sérsic index to artificially higher values.

Now we consider the opposite scenario: what is the effect of including bars in the fit to Equation (1), instead of excluding them (or parameterizing them). For this test we simply refit all profiles of galaxies in which data have been removed from the fit including the whole range in $r$ (yet still excluding nuclei). The results are shown in Figure 19. In all panels, a one-to-one correlation is shown by a dashed line. It is no surprise that the rms deviation (left panel) increases when we include those deviations. This is shown in the bottom panel of

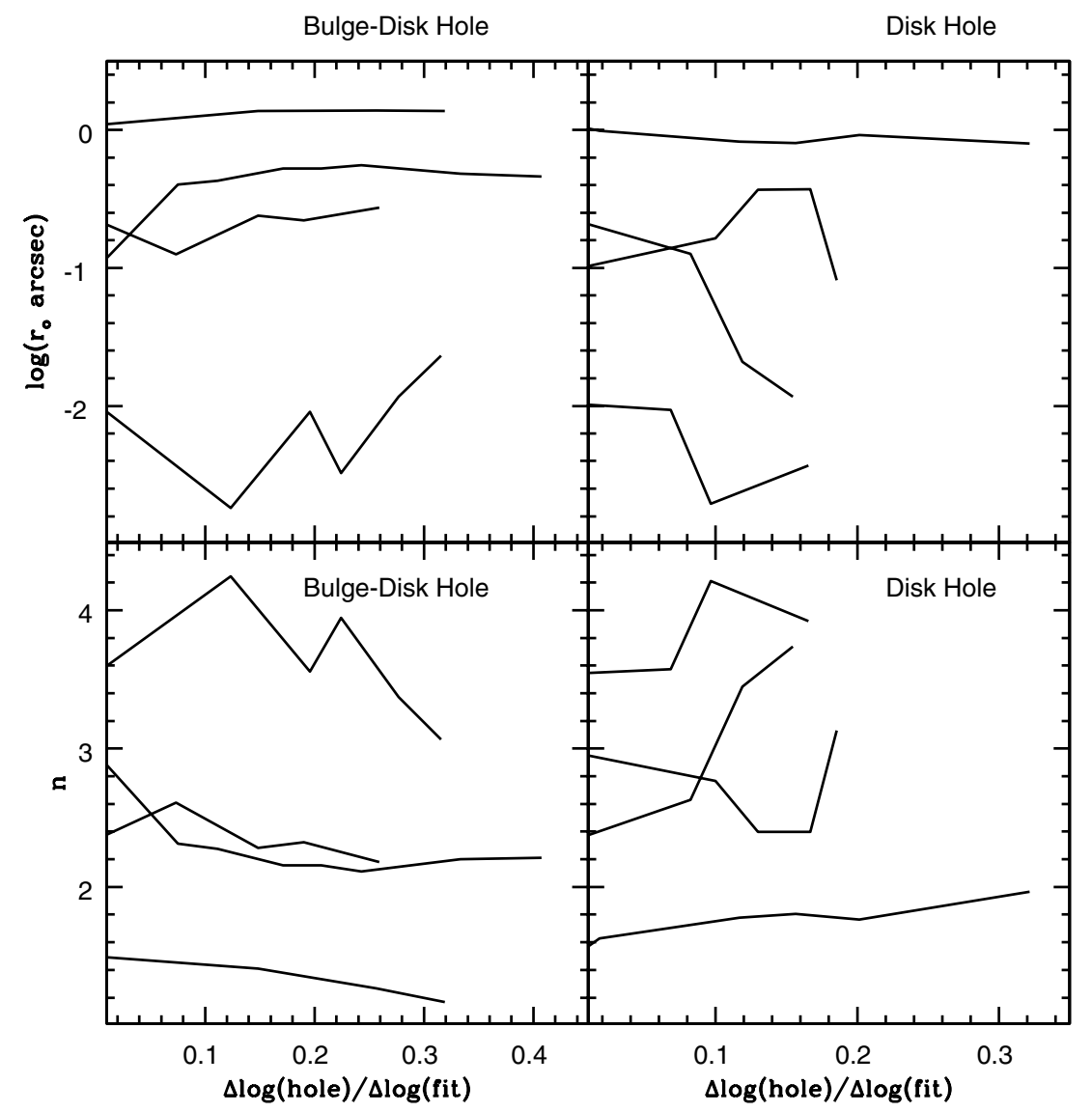

Figure 18. Each profile shows the effect of removing data from a profile and then fitting Equation (1) to the profile. The innermost point in each profile is the best fit to the galaxy with no points removed. The profiles shown on the left are meant to mimic the effect of those distortions to the bulge-disk transition region, and the profiles on the right are meant to mimic those distortions only affecting the outer disk. 

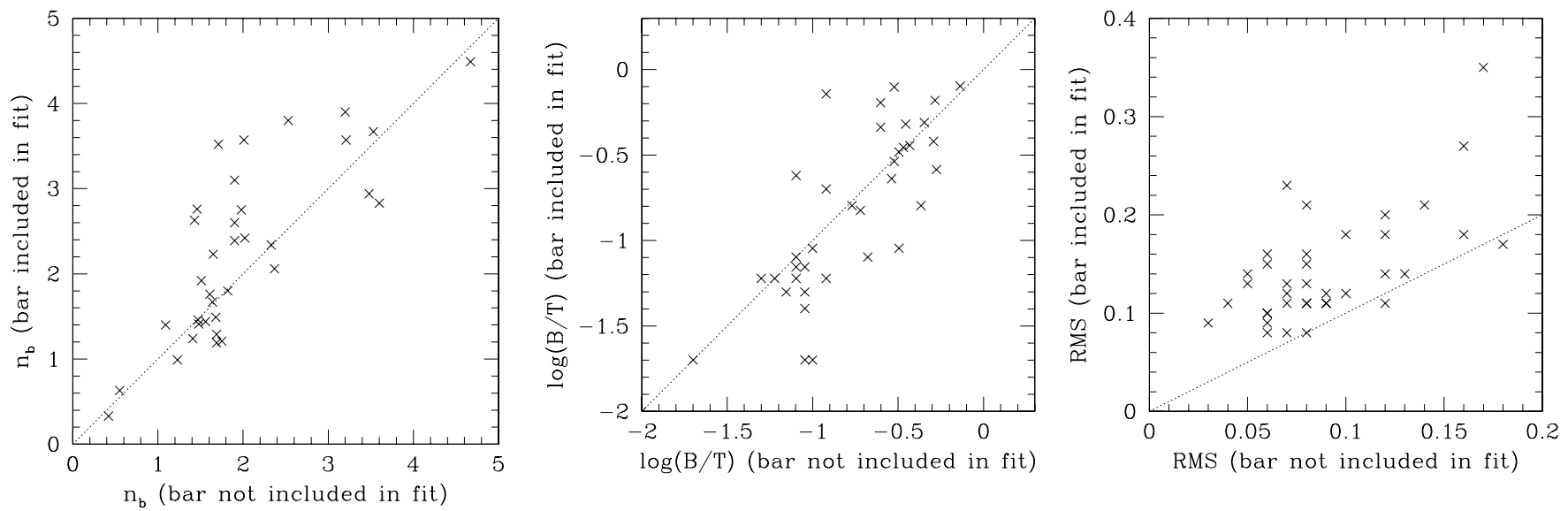

Figure 19. Left panel: comparison of bulge Sérsic index for those galaxies in which a bar is removed from the fit to those in which the bar is included in the fit. Middle panel: the same but comparing the bulge-to-total luminosity ratio. Right panel: the same but comparing the rms deviation of the fit. In all plots the dashed line represents a one-to-one correlation.

Figure 19. The typical deviation in which distortions are omitted is $0.09 \mathrm{mag} \operatorname{arcsec}^{-2}$, yet it increases to $0.14 \mathrm{mag} \operatorname{arcsec}^{-2}$ when the distortion is included. The bulge-to-total luminosity ratio appears relatively robust (middle panel). Finally, the Sérsic index (right panel) is definitely affected by including bars and other distortions in the fit. The typical effect of including bars in the fit is to increase the Sérsic index. This is no surprise, since more light is being included at radii beyond the half-light radius of the bulge. The effects of increasing Sérsic index and a modest increase in scatter of $B / T$ are more pronounced in higher $B / T$ and higher Sérsic index bulges.

\section{REFERENCES}

Adelman-McCarthy, J. K., et al. 2006, ApJS, 162, 38

Aguerri, J. A. L., Balcells, M., \& Peletier, R. F. 2001, A\&A, 367, 428

Allen, P. D., Driver, S. P., Graham, A. W., Cameron, E., Liske, J., \& de Propris, R. 2006, MNRAS, 371, 2

Andredakis, Y. C. 1998, MNRAS, 295, 725

Andredakis, Y. C., Peletier, R. F., \& Balcells, M. 1995, MNRAS, 275, 874

Andredakis, Y. C., \& Sanders, R. H. 1994, MNRAS, 267, 283

Balcells, M., Graham, A. W., Domínguez-Palmero, L., \& Peletier, R. F. 2003, ApJ, 582, L79

Bender, R., Burstein, D., \& Faber, S. M. 1992, ApJ, 399, 462

Bender, R., \& Moellenhoff, C. 1987, A\&A, 177, 71

Böker, T., Laine, S., van der Marel, R. P., Sarzi, M., Rix, H.-W., Ho, L. C., \& Shields, J. C. 2002, AJ, 123, 1389

Caon, N., Capaccioli, M., \& D’Onofrio, M. 1994, A\&AS, 106, 199

Carollo, C. M. 1999, ApJ, 523, 566

Carollo, C. M., Stiavelli, M., de Zeeuw, P. T., \& Mack, J. 1997, AJ, 114, 2366

Carollo, C. M., Stiavelli, M., Seigar, M., de Zeeuw, P. T., \& Dejonghe, H. 2002, AJ, 123, 159

Cheng, K. P., Collins, N., Angione, R., Talbert, F., Hintzen, P., Smith, E. P., \& Stecher, T., The UIT Team (eds.) 1997, UV/Visible Sky Gallery on CD-ROM Courteau, S., de Jong, R. S., \& Broeils, A. H. 1996, ApJ, 457, L73

de Jong, R. S. 1996, J. Astron. Data, 2, 1

de Jong, R. S., Simard, L., Davies, R. L., Saglia, R. P., Burstein, D., Colless, M., McMahan, R., \& Wegner, G. 2004, MNRAS, 355, 1155

de Vaucouleurs, G., de Vaucouleurs, A., Corwin, H. G., Jr., Buta, R. J., Paturel, G., \& Fouque, P. 1991, Third Reference Catalogue of Bright Galaxies, Vols. 1-3 (chap. XII, 2069 pp, 7 figures) (Berlin: Springer)

Debattista, V. P., Carollo, C. M., Mayer, L., \& Moore, B. 2004, ApJ, 604, L93

Djorgovski, S., \& Davis, M. 1987, ApJ, 313, 59

D’Onghia, E., \& Burkert, A. 2004, ApJ, 612, L13

Dressler, A. 1980, ApJ, 236, 351

Dressler, A., Lynden Bell, D., Burstein, D., Davies, R. L., Faber, S. M., Terlevich, R., \& Wegner, G. 1987, ApJ, 313, 42

Drory, N., \& Fisher, D. B. 2007, ApJ, 664, 640

Eliche-Moral, M. C., Balcells, M., Aguerri, J. A. L., \& González-García, A. C. 2006, A\&A, 457, 91
Erwin, P., Beckman, J. E., \& Vega-Beltran, J.-C. 2004, in Astrophysics and Space Science Library 319, Astrophysics and Space Science Library, ed. D. L. Block, I. Puerari, K. C. Freeman, R. Groess, \& E. K. Block, 775

Erwin, P., \& Sparke, L. S. 2002, AJ, 124, 65

Erwin, P., \& Sparke, L. S. 2003, ApJS, 146, 299

Faber, S. M., Wegner, G., Burstein, D., Davies, R. L., Dressler, A., Lynden Bell, D., \& Terlevich, R. J. 1989, ApJS, 69, 763

Falcón-Barroso, J., Peletier, R. F., \& Balcells, M. 2002, MNRAS, 335, 741

Fathi, K., \& Peletier, R. F. 2003, A\&A, 407, 61

Fisher, D. B. 2006, ApJ, 642, L17

Frei, Z., Guhathakurta, P., Gunn, J. E., \& Tyson, J. A. 1996, AJ, 111, 174

Friedli, D. 1999, in Astronomical Society of the Pacific Conference Series 187, The Evolution of Galaxies on Cosmological Timescales, ed. J. E. Beckman, \& T. J. Mahoney, 88

Graham, A. W. 2001, AJ, 121, 820

Graham, A. W., \& Colless, M. 1997, MNRAS, 287, 221

Graham, A. W., \& Driver, S. P. 2005, PASA, 22, 118

Graham, A. W., Lauer, T. R., Colless, M., \& Postman, M. 1996, ApJ, 465,534

Helfer, T. T., Thornley, M. D., Regan, M. W., Wong, T., Sheth, K., Vogel, S. N., Blitz, L., \& Bock, D. C.-J. 2003, ApJS, 145, 259

Hohl, F. 1975, in IAU Symp. 69: Dynamics of the Solar Systems, ed. A. V. Oppenheim, \& R. W. Schafer, 349

Holtzman, J. A., Burrows, C. J., Casertano, S., Hester, J. J., Trauger, J. T., Watson, A. M., \& Worthey, G. 1995, PASP, 107, 1065

Jogee, S., Scoville, N., \& Kenney, J. D. P. 2005, ApJ, 630, 837

Kannappan, S. J., Jansen, R. A., \& Barton, E. J. 2004, AJ, 127, 1371

Kennicutt, R. C., Jr., et al. 2003, PASP, 115, 928

Kent, S. M. 1985, ApJS, 59, 115

Khosroshahi, H. G., Wadadekar, Y., \& Kembhavi, A. 2000, ApJ, 533, 162

Knapen, J. H., Stedman, S., Bramich, D. M., Folkes, S. L., \& Bradley, T. R. 2004, A\&A, 426, 1135

Koda, J., Milosavljevic, M., \& Shapiro, P. R. 2007, arXiv:0711.3014

Kormendy, J. 1977, ApJ, 218, 333

Kormendy, J. 1982, in Saas-Fee Advanced Course 12: Morphology and Dynamics of Galaxies Saas-Fee 12, Morphology and Dynamics of Galaxies, 113

Kormendy, J. 1993, in IAU Symp. 153: Galactic Bulges, 209

Kormendy, J., Fisher, D. B., Cornell, M. E., \& Bender, R. 2008, ApJS, submitted

Kormendy, J., \& Kennicutt, R. C. 2004, ARA\&A, 42, 603

Kuchinski, L. E., et al. 2000, ApJS, 131, 441

Larsen, S. S., \& Richtler, T. 1999, A\&A, 345, 59

Lauer, T. R., et al. 1995, AJ, 110, 2622

Lauer, T. R., et al. 2005, AJ, 129, 2138

MacArthur, L. A., Courteau, S., Bell, E., \& Holtzman, J. A. 2004, ApJS, 152,175

MacArthur, L. A., Courteau, S., \& Holtzman, J. A. 2003, ApJ, 582, 689

Mastropietro, C., Moore, B., Mayer, L., Debattista, V. P., Piffaretti, R., \& Stadel, J. 2005, MNRAS, 364, 607

Moellenhoff, C., Matthias, M., \& Gerhard, O. E. 1995, A\&A, 301, 359 
Möllenhoff, C., \& Heidt, J. 2001, A\&A, 368, 16

Moore, B., Katz, N., Lake, G., Dressler, A., \& Oemler, A., Jr. 1996, Nature, 379,613

Noguchi, M. 1999, ApJ, 514, 77

Peeples, M. S., \& Martini, P. 2006, ApJ, 652, 1097

Peletier, R. F., \& Balcells, M. 1996, AJ, 111, 2238

Pfenniger, D., \& Friedli, D. 1991, A\&A, 252, 75

Pfenniger, D., \& Norman, C. 1990, ApJ, 363, 391

Regan, M. W., Thornley, M. D., Helfer, T. T., Sheth, K., Wong, T., Vogel, S. N., Blitz, L., \& Bock, D. C.-J. 2001, ApJ, 561, 218

Saglia, R. P., Bertschinger, E., Baggley, G., Burstein, D., Colless, M., Davies, R. L., McMahan, R. K. Jr., \& Wegner, G. 1997, ApJS, 109, 79

Sandage, A. 1961, The Hubble Atlas of Galaxies (Washington: Carnegie Institution)

Sandage, A., \& Bedke, J. 1994, The Carnegie Atlas of Galaxies (Washington, DC: Carnegie Institution of Washington with The Flintridge Foundation)

Scarlata, C., et al. 2004, AJ, 128, 1124
Schlegel, D. J., Finkbeiner, D. P., \& Davis, M. 1998, ApJ, 500, 525

Scorza, C., \& Bender, R. 1995, A\&A, 293, 20

Sérsic, J. L. 1968, Atlas de galaxias australes (Cordoba, Argentina: Obs. Astronomico)

Sheth, K., Vogel, S. N., Regan, M. W., Thornley, M. D., \& Teuben, P. J. 2005, ApJ, 632, 217

Simkin, S. M., Su, H. J., \& Schwarz, M. P. 1980, ApJ, 237, 404

Sirianni, M., et al. 2005, PASP, 117, 1049

Skrutskie, M. F., et al. 2006, AJ, 131, 1163

Smith, J. A., et al. 2002, AJ, 123, 2121

Stewart, K. R., Bullock, J. S., Wechsler, R. H., Maller, A. H., \& Zentner, A. R. 2007, arXiv:711.5027

Thomas, D., \& Davies, R. L. 2006, MNRAS, 366, 510

Tully, R. B., \& Fisher, J. R. 1988, Catalog of Nearby Galaxies (Cambridge: Cambridge Univ. Press), 224

van Albada, T. S. 1982, MNRAS, 201, 939

van den Bergh, S. 1976, ApJ, 206, 883 